\title{
Origins and Applications of London Dispersion Forces and Hamaker Constants in Ceramics
}

\author{
Roger H. French ${ }^{\star, \star \star}$ \\ Central Research, DuPont Company, Wilmington, Delaware 19880; and Materials Science Department, \\ University of Pennsylvania, Philadelphia, Pennsylvania 19104
}

The London dispersion forces, along with the Debye and Keesom forces, constitute the long-range van der Waals forces. London's and Hamaker's work on the point-to-point dispersion interaction and Lifshitz's development of the continuum theory of dispersion are the foundations of our understanding of dispersion forces. Dispersion forces are present for all materials and are intrinsically related to the optical properties and the underlying interband electronic structures of materials. The force law scaling constant of the dispersion force, known as the Hamaker constant, can be determined from spectral or parametric optical properties of materials, combined with knowledge of the configuration of the materials. With recent access to new experimental and $a b$ initio tools for determination of optical properties of materials, dispersion force research has new opportunities for detailed studies. Opportunities include development of improved index approximations and parametric representations of the optical properties for estimation of Hamaker constants. Expanded databases of London dispersion spectra of materials will permit accurate estimation of both nonretarded and retarded dispersion forces in complex configurations. Development of solutions for generalized multilayer configurations of materials are needed for the treatment of more-complex problems, such as graded interfaces. Dispersion forces can play a critical role in materials applications. Typically, they are a component with other forces in a force balance, and it is this balance that dictates the resulting behavior. The ubiquitous nature of the London dispersion forces makes them a factor in a wide spectrum of problems; they have been in evidence since the pioneering work of Young and Laplace on wetting, contact angles, and surface energies. Additional applications include the interparticle forces that can be measured by direct techniques, such as atomic force microscopy. London dispersion forces are important in both adhesion and in sintering, where the detailed shape at the crack tip and at the sintering neck can be controlled by the dispersion forces. Dispersion forces have

-D. R. Clarke

Manuscript No. 188760. Received February 1, 2000; approved June 5, 2000.

${ }^{\star}$ Member, American Ceramic Society.

${ }^{\star *}$ Fellow, American Ceramic Society.

\section{Article Table of Contents}

Introduction 2118

Overview 2118

History 2118

Structure of This Article 2119

Origins of Dispersion Forces 2119

van der Waals Forces 2119

Lifshitz Theory and Electronic Structure and Bonding 2121

Full Spectral Nonretarded Hamaker Constant $A^{\mathrm{NR}}$ from Spectral Optical Properties 2125

Methods for Estimating Hamaker Constant $A^{\mathrm{NR}} 2128$

Retardation of Dispersion Forces and the Retarded Hamaker Constant $A^{\mathrm{R}} 2129$

Applications 2129

Classes of Materials 2130

Surface Energies, Wetting, and Contact Angles 2130

Balance of Forces 2132

Colloidal Systems 2132

Interparticle Forces 2132

Adhesion and Sintering 2133

Equilibrium Intergranular and Surficial Films 2135

Retardation of Dispersion Forces 2139

Conclusions 2141

Acknowledgements 2142

References 2142

an important role in the properties of numerous ceramics that contain intergranular films, and here the opportunity exists for the development of an integrated understanding of intergranular films that encompasses dispersion forces, segregation, multilayer adsorption, and structure. The intrinsic length scale at which there is a transition from the continuum perspective (dispersion forces) to the atomistic perspective (encompassing interatomic bonds) is critical in many materials problems, and the relationship of dispersion forces and intergranular films may represent an important opportunity to probe this topic.

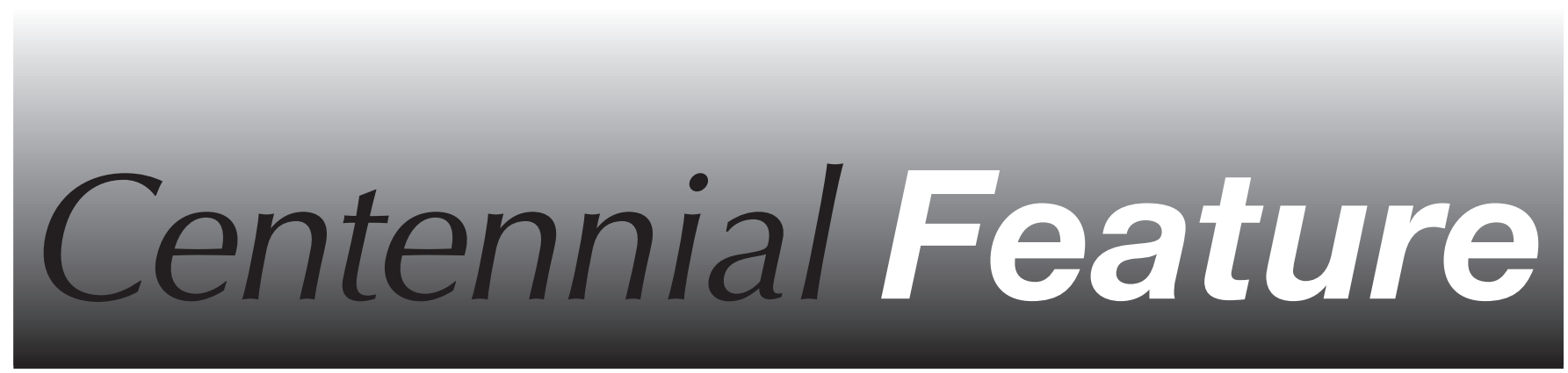


The London dispersion force is retarded at large separations, where the transit time of the electromagnetic interaction must be considered explicitly. Novel phenomena, such as equilibrium surficial films and bimodal wetting/dewetting, can result in materials systems when the characteristic wavelengths of the interatomic bonds and the physical interlayer thicknesses lead to a change in the sign of the dispersion force. Use of these novel phenomena in future materials applications provides interesting opportunities in materials design.

\section{Introduction}

$\mathrm{T}$ HE origins and applications of the van der Waals (vdW) force and its major component, the London dispersion (LD) force, have been of continuous interest for more than 200 years, spanning virtually all areas of science from mathematics to physics, chemistry, and biology. Dispersion forces are manifest throughout the world. Examples are the wetting of solids by liquids, capillarity of liquids, structure of biological cell walls, flocculation of colloidal suspensions, adhesion and fracture of materials, and numerous other microscopic and macroscopic phenomena.

\section{(1) Overview}

Research in dispersion forces has elucidated their role in many practical application areas and phenomena. Our fundamental understanding of dispersion forces mostly derives from the work of Lifshitz, and, in the 50 years since his work, we have expanded the foundation of dispersion force science and advanced its application to real world problems, such as wetting and colloidal processing.

Improved spectral data, numerical methods, and advances in direct force measurements have occurred during the past 15 years. The spectral data allow the direct determination of the Hamaker constant $(A)$ for the dispersion force from first-principles Lifshitz theory. This, combined with the ability to directly measure these forces using atomic force microscopy, has produced a renaissance of research activity. Dispersion force theory is now applied to such areas as sintering and crack propagation and to the role of intergranular glassy films in ceramics. Our improved understanding of dispersion forces has advanced our understanding of room-temperature colloidal processing of ceramics and hightemperature processing of ceramics exhibiting intergranular films. We also understand better the design and performance of structural and electronic ceramics from viscous-sintered systems exhibiting intergranular films.

Dispersion forces have such wide application that they are intrinsically interdisciplinary in nature, spanning scientific and industrial applications and all fields of science. As advances have occurred in one area of science, they have enabled advances in other apparently unrelated fields that are united only by the underlying role played by dispersion forces. It is for this reason that we undertake a detailed view of the history, current state, and future directions of our understanding of the LD forces.

\section{(2) History}

One of the earliest references to the wetting of solids by liquids can be found in the observations of Taylor and of Hauksbee, who, in the early 1700 s, qualitatively described the shape of a fluid interface that wets a vertical plane. ${ }^{1}$ In the early 1800 s, many scientists were interested in the behavior of liquids and, in particular, what aspects of liquids are responsible for their wetting, capillarity, and the various contact angles that are apparent when alternate combinations of substrates and liquids are examined. Among the first works that considered phenomena in which vdW forces can play a critical role was by Young, ${ }^{2}$ in 1805, in his "An Essay on the Cohesion of Fluids," in which he postulated what has become known as the Young-Laplace equation for wetting. ${ }^{3}$ The discussion of capillary action went on actively, including efforts by LaPlace, Gauss, Maxwell, and Thomson. ${ }^{4}$ It was van der Waals ${ }^{5}$ who, in 1893, developed a thermodynamic theory of capillarity for application to the behavior of liquids and who established the minimization of free energy as the criterion for equilibrium in a liquid-gas system and then applied this to surface tensions. van der Waals introduced the long-range vdW forces as resulting from dipole and quadrapolar interactions between molecules that make up gases, liquids, or solids. van der Waals' work followed Gibbs' thermodynamic work on capillarity entitled "On the Equilibrium of Heterogeneous Substances."6,7

Surface tension represents the effects of vdW forces of a material on itself, effectively a self energy, producing a surface tension at the interface between the material and the gas phase. London, in $1930,{ }^{8-11}$ using the advances of the then new quantum mechanics, was able to demonstrate that the vdW forces resulting from permanent dipoles were not the most universal, because many materials do not have permanent dipoles, and because permanent dipole interactions decrease dramatically with increasing temperature. London showed that it is transient-induced dipoles that result in the dispersion forces; induced dipoles result from the intrinsic polarizability of the interatomic bonds and the presence of a propagating electromagnetic field and are long range and do not disappear at high temperatures. It was from this work that Hamaker ${ }^{12}$ extended the understanding of $\mathrm{LD}$ forces by summing the point-by-point interactions among molecules and producing a measure of the net attraction of two separate bodies. Hamaker was very active in the 1930 s and introduced the dispersion interaction between bodies, leading to the development of the Hamaker constant $A$ to establish the magnitude of the dispersion forces.

Even with much progress through the 1930s, the fundamental relationship between a material's properties and its LD forces was not clear. The concept existed of transient dipoles that were induced in one molecule's interatomic bonds by the propagating electromagnetic field produced by the interatomic bonds in adjacent molecules. Because this transient-induced dipole interaction could be associated with the zero point motion of a quantum mechanical oscillator whose energy could not be dissipated, it was realized that the propagating electromagnetic interaction from one molecule to another was also nondissipative, even as it induced a polarization interaction. (As shown later, instead of the energy release associated with a dissipative interaction, this LD interaction results instead from the exchange of virtual photons.)

It was from this perspective- that the interaction propagated as an induced electromagnetic wave-that eventually brought the fundamental properties of materials into consideration with the dispersion forces. For example, Sellmeier, ${ }^{13}$ in 1872 , published a series of papers entitled "Regarding the Sympathetic Oscillations Excited in Particles by Oscillations of the Ether and Their Feedback to the Latter, Particularly as a Means of Explaining Dispersion and Its Anomalies." The dispersion referred to here is the wavelength or frequency dependence of a material's response to electromagnetic radiation. Sellmeir's research was closely coupled to the development of Maxwell's wave equations for electromagnetic radiation. This work by Maxwell and the associated group of scientists, sometimes referred to as the Maxwellians, ${ }^{14}$ culminated in the 1880 s with Heaviside's ${ }^{15}$ compact statement of Maxwell's equations. It is the close relationship of the optical properties of materials, the propagation of electromagnetic waves in materials (as governed by Maxwell's equations), and the dispersion of these waves as they travel through a material that ties the dispersion forces to the optical properties and electronic structure of materials. However, even by the time of Hamaker's 1930s work, this connection was not yet made.

In 1948, Casimir ${ }^{16}$ published a seminal paper on quantum electrodynamics (QED) ${ }^{17}$ entitled, "On the Attraction of Two Perfectly Conducting Plates," in which he used QED to calculate the LD force between two metallic plates. This particular dispersion force is now called the Casimir force and has been experimentally confirmed only recently by Lamoreaux. ${ }^{18}$ Lifshitz then followed, in $1956,{ }^{19}$ in $1960,{ }^{20}$ and in $1961^{21}$ with the general QED solution (including the effects of retardation) for the LD forces of two bodies separated by an interlayer material, in which the optical properties of the bulk materials, as represented by the dielectric function at optical frequencies, were used to directly 
calculate the dispersion force. The Lifshitz theory represented a major advance in the field of dispersion forces because it allowed the calculation of dispersion forces given known optical properties.

Dispersion force research from the 1960s to the 1980s focused on determining parametric representations of the optical properties of materials for use in dispersion force calculations based on Lifshitz theory. Moreover, much work was done using surface force apparatus $^{22}$ to measure intersurface forces between solids. Knowledge of dispersion forces had a major impact on the theory of interparticle interactions in colloidal systems and in wetting. More recently, vacuum ultraviolet (VUV) spectroscopy ${ }^{23}$ and valence-electron energy-loss spectroscopy ${ }^{24}$ have provided the full spectral optical properties over the wide energy range needed for Lifshitz calculations, thereby enabling the direct determination of dispersion forces from optical properties for solid systems. Also, the use of atomic force microscopy to measure force-distance curves in numerous systems has led to studies of the dispersion forces for diverse materials systems.

There have been many excellent articles ${ }^{25,26}$ and books ${ }^{27-29}$ that review the origins and determination of the dispersion forces, calculation of Hamaker constants, ${ }^{30}$ and the applications of dispersion forces in various fields. ${ }^{31-34}$

\section{(3) Structure of This Article}

$\mathrm{vdW}$ forces are long-range forces that can play a critical role in the interaction of materials. At the same time, they are relatively weak forces and can be overwhelmed by other shorter-range but larger forces. It is, therefore, essential to be able to evaluate the relative role and importance of dispersion forces in problems involving various classes of materials. We provide an overview of dispersion forces with an emphasis on applications in ceramics involving inorganic materials. We also examine some polymeric and metallic materials that might be used in ceramics processing or ceramic applications.

We begin with a discussion of the origin of the dispersion forces in the electronic structure and optical properties of materials. We next assess the methods for direct determination of Hamaker constants of the dispersion forces using Lifshitz theory and full spectral data and then methods of estimating Hamaker constants from parametric optical data and simple index of refraction relations. We then look at the effect of retardation of the dispersion forces at large separations, which result because of the finite speed of light, and how these retarded forces also can be calculated using Lifshitz theory.

We review the application of dispersion forces in colloidal systems, interface energies, and wetting. We next study the intersurface forces resulting from the dispersion interactions and the role of dispersion forces in adhesion and sintering. We present the mechanical and electrical properties of a broad range of ceramic systems that exhibit intergranular glassy films, mindful of the importance these films have acquired in the past 15 years for our understanding of ceramics processing and properties. We then finish with a discussion of the effects of retardation on wetting, which can lead to unique wetting conditions. During our presentation, we highlight various topics that represent opportunities for further research.

\section{Origins of Dispersion Forces}

The long-range vdW forces between molecules in materials result from the interactions of dipoles. We first consider, in Section $\mathrm{II}(1)(\mathrm{A})$, the interactions that produce the vdW forces as simple point-to-point interactions between dipoles so as to highlight the nature of the individual interaction. These dipoles can be considered as the individual dipoles present in a molecule or as the electronic orbitals of an atom or a molecule. To produce a macroscopic attraction from these dipole interactions, the summation of the dipolar attractions over the atoms or molecules in a bulk material is required, and this is discussed in Section II(1)(B).

\section{(1) van der Waals Forces}

(A) Point-to-Point Dipole Interactions for Molecules: To determine the vdW force between two molecules, let us first consider each uncharged molecule as consisting of a permanent or induced dipole. The vdW forces can be considered as resulting from three additive terms, the Keesom force, the Debye force, and the LD force, as shown in Eq. (1).

$$
F_{\mathrm{vdW}} \approx F_{\text {Keesom }}+F_{\text {Debye }}+F_{\text {LD }}
$$

Parsegian $^{25}$ has written an intuitive discussion on the fundamental nature of induced dipole interactions and the Debye induction and LD interactions of the long-range $\mathrm{vdW}$ forces. Beyond the two permanent dipoles that result in the Keesom force, induced dipoles play a much larger role in the vdW forces. Dipole moments can be induced in all atoms and molecules and, therefore, are not restricted to atoms or molecules that have permanent dipole moments.

(a) Keesom Force-Orientation Effect: In a system of two permanent dipoles, such as $\mathrm{H}_{2} \mathrm{O}$ molecules, the interaction of the dipole's electric fields results in either an attractive force, when the dipoles are antiparallel, or a repulsive force, when they are parallel. This permanent dipole orientation effect results in a force that Keesom $^{35-38}$ first discussed in 1912. The Keesom force can be attractive or repulsive, but vanishes as temperature increases, because thermally induced motions of the permanent dipoles disorder their mutual alignment.

(b) Debye Force-Induction Effect: Permanent dipole moments interacting with any atom or molecule can exhibit induced dipole moments. These result from the intrinsic polarizability of the multiple electron clouds that constitute the interatomic bonds of the atom or molecule. This polarizability causes the atom to respond to an applied electromagnetic field. This can be a static electric field or, more typically, an electric field that is varying at the infrared (IR) frequency of a molecular vibration of the permanent dipole. It also can be the electromagnetic field of a photon of light that fluctuates at optical frequencies. For time- or frequency-dependent electromagnetic fields, the polarization response of the electron cloud (i.e., the interatomic bond) also is frequency dependent. Because the electron cloud has a characteristic frequency that spans the ultraviolet (UV) and visible frequencies, the induced polarization of the electron cloud is responsive to the imposed electromagnetic field, and it has a much faster response time to changes in the inducing field. In other words, electron clouds with induced dipoles have much higher characteristic response frequencies than the very-low-frequency oscillations or rotations of permanent molecular dipoles. In 1920, Debye $\mathrm{e}^{39,40}$ proposed that a temperature-independent dipolar interaction was possible for a single permanent dipole and its induced dipoles: The electromagnetic field of a single permanent dipole induces a dipole moment in the electron cloud of another atom or molecule. These induced high-frequency electronic dipole moments can couple to the lower-frequency oscillations of the permanent dipolar molecule. The interaction energy for this permanent-dipole/induced-dipole interaction results in the Debye force. This Debye force does not disappear at high temperature and is always attractive. The Debye force requires the presence of at least one permanent dipole and, therefore, is not universally present for all atoms or molecules.

(c) London Force-Dispersion Effect: London $^{8}$ pointed out that the Keesom and Debye forces cannot be solely or universally responsible for the $\mathrm{vdW}$ forces, because these forces require the presence of permanent dipole moments; however, many molecules that have no permanent dipole moments exhibit long-range vdW forces. An example London cites are the rare gases that have negligible dipole and quadropole moments; however, they have $\mathrm{vdW}$ forces that are 100 times larger than can be accounted for by the Keesom and Debye forces. It was at this point that London introduced the idea that interatomic bonds in atoms and molecules can themselves induce dipole moments in nearby interatomic bonds. The characteristic frequency of interatomic bonds is in the UV and optical frequency ranges, and the bonds can produce 
propagating electromagnetic waves or photons. The typical way that photons are created is when an electron in an excited state reduces its energy and moves to a lower-energy state, thereby releasing a propagating electromagnetic wave, or photon, whose energy is equal to the energy lost by the electronic transition. An example of this is the production of light that occurs when a material is heated to a high temperature and then produces radiant light.

London did not focus on the active production of propagating electromagnetic waves that result when an electron drops to its ground state. This active production of light is always associated with a loss of energy and, therefore, is not the basis of a universal $\mathrm{vdW}$ interaction. Instead, London utilized an idea from the new quantum mechanics, which stated that an electron, even in its ground state and at absolute zero temperature, universally exhibits a zero point motion. This zero point motion of the electron, which corresponds to the fluctuations of the electron cloud, occurs at the characteristic frequency of the electron that is in the optical range, and this ground state energy cannot be dissipated. This zero point motion of the electron results in a propagating electromagnetic wave that also cannot be dissipated, and we refer to such waves as virtual photons so as to distinguish them from energy-dissipating photons produced from the transition from an excited state to the ground state.

Similar to the zero point motion of any electron on any atom producing electromagnetic waves in the form of virtual photons, these electromagnetic waves and their associated fields can induce dipole moments in the electron clouds of nearby atoms or molecules, just as do the electromagnetic fields from permanent dipoles. It is these induced-dipole/induced-dipole interactions that result in an attractive force that is the basis of the $\mathrm{LD}$ force. The dispersion force results between individual interatomic bonds that interact by the exchange of virtual photons, and the dispersion effect is additive for all the interatomic bonds in atoms or molecules. The dispersion force is present for all atoms and molecules and contributes to the vdW force for all materials: It is a universal contributor to the vdW force.

The LD interaction of two induced dipoles obeys a power-law relationship with a $L^{-6}$ power for the LD interaction energy (as shown in Eq. (2)) and a $L^{-7}$ power for the LD force (as shown in Eq. (3)), where $L$ is the separation distance between the dipoles. The power-law relationship also exists for the LD interaction of two materials, but the exponent, as given in Table I, is different and is discussed in more detail in Section II(1)(B).

$$
\begin{aligned}
& E_{\mathrm{vdW}}(d) \propto \frac{A_{\text {Hamaker }}}{L^{m}} \\
& F_{\mathrm{vdW}}(d) \propto \frac{A_{\text {Hamaker }}}{L^{m+1}}
\end{aligned}
$$

(B) Continuum Approach for Bulk Interactions: Once the nature of the individual dipolar interactions was identified, Hamaker $^{12}$ was able to determine the macroscopic London-vdW interaction between two spherical particles by summing all dipolar interactions of the atoms and molecules of a solid or liquid. De Boer $^{41}$ calculated the vdW interaction for parallel plane solids, also by summation of dipolar interactions. This method is based on the assumption of pairwise additivity of the LD interaction, which is always met by two bodies under vacuum with no intervening phase between them (as considered by Hamaker). As presented in Section II(2), when there is an intervening or interlayer material

Table I. London Dispersion Power Laws for Various Configurations

\begin{tabular}{lccc}
\hline \multicolumn{1}{c}{ Configuration } & $m$ & Energy & Force \\
\hline Molecules: point to point & 6 & 6 & 7 \\
Two-plane parallel bodies & 2 & 2 & 3 \\
Two spherical particles & 1 & 1 & 2 \\
\hline
\end{tabular}

present between the two bodies, the simple assumption of additivity may break down.

Let us now consider in detail the formalism developed by Hamaker for nonretarded vdW interactions between materials. (The behavior of retarded vdW interactions is discussed in detail in Section II(2)(E).) Consider the configuration for two plane-parallel materials (numbered 1 and 3) shown in Fig. 1(a) that are separated a distance $L$ by an interlayer (numbered 2) that may be either a vacuum or another material. The Hamaker constant, $A^{\mathrm{NR}}$, represents the magnitude of the LD interactions and is defined by Eq. (4) for the interaction energy and by Eq. (5) for the force between two materials. $A^{\mathrm{NR}}$ is defined by convention as being positive for an attractive force corresponding to a negative LD interaction energy. $A^{\mathrm{NR}}$ is only a function of the material properties of the two particles and the interlayer material, assuming a uniform interlayer film.

$$
\begin{aligned}
& A_{123}^{\mathrm{NR}}=-12 \pi L^{2} E_{\mathrm{vdW}} \\
& A_{123}^{\mathrm{NR}}=-6 \pi L^{3} F_{\mathrm{vdW}}
\end{aligned}
$$

This LD interaction is considered long range, because, across a uniform gap between plane-parallel materials, the interaction energy varies as a simple power law with $L^{-2}$, and the force varies with $L^{-3}$, where $L$ is the gap thickness. Most other forces found in physical systems may be of much larger magnitude, but decrease much more rapidly with distance.

The three-layer configurations shown in Fig. 1(a) can apply to many materials systems of interest in ceramics. For example, if there is a vacuum or an intergranular glassy film between two particles of the same material-i.e., material 1 and 3 are identical-then we have a symmetrical configuration denoted as 121 . If, instead, the two particles are dissimilar-i.e., material 1 is separated from material 3 by an interlayer of material 2-then we have an asymmetrical configuration denoted as 123. An additional 123 configuration is the case of a film wetting a free surface, where material 3 would be vacuum or air.

Ninham and Parsegian ${ }^{42}$ solved the LD interaction for the more-complex five-layer configuration, shown in Fig. 1(b), which is appropriate to a soap-bubble film. In this symmetrical case, denoted as 12321 , material 1 is air, while the three-layer interlayer is the soap bubble membrane and consists of a surfactant layer of material 2, which coats the liquid-water layer, denoted as material 3. For the soap film, it is assumed that the surfactant layer has a fixed thickness, and when the thickness of the film is varied, only the thickness $a$ of material 3 changes, leading to $a$ scaling for this $A_{12321}^{\mathrm{NR}}$ Hamaker constant. If the real variation in the system was such that material 2 and material 3 would vary in thickness, thereby keeping the relative quantities of materials 2 and 3 in the interlayer constant, then the $A_{12321}^{\mathrm{NR}}$ Hamaker constant is considered to be on an $L$-scaling basis and can be completely different from

(a)

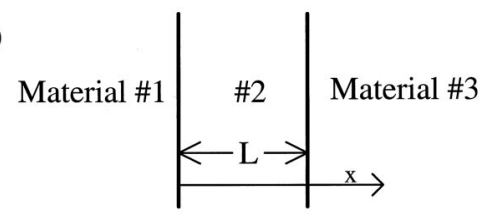

(b)

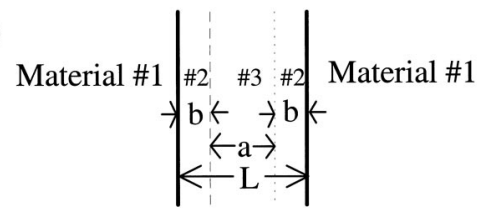

Fig. 1. Schematic geometry for Hamaker constant calculations. (a) Corresponds to asymmetrical 1-2-3 Hamaker constants, where, if materials 1 and 3 are the same, then this is the simpler case of a symmetrical 1-2-1 Hamaker constant. (b) Represents the more-complex symmetrical fivelayer case, where a layer of material 2 forms on the surface of material 1 while material 3 is the central phase in the interfacial film. (Reprinted with permission from Elsevier Science. ${ }^{23}$ ) 
the $a$-scaling Hamaker constant. The details of the scaling changes in the $A_{12321}^{\mathrm{NR}}$ Hamaker constants are discussed in the paper by French et al. ${ }^{23}$ Parsegian and Ninham ${ }^{43}$ also solved the more general problem for films with more layers than the 12321 case, and this is discussed in more detail in Section III(7)(D).

(C) Zero Frequency, IR, and UV Terms in LD Interactions: The vdW force has three components: the Keesom, or orientation force; the Debye, or induction force; and the LD force. We can partition these contributions to the Hamaker constant of the vdW force, as shown in Eq. (6), so as to evaluate the magnitudes of the differing dipolar contributions.

$$
A_{\mathrm{vdW}} \approx A_{\text {Keesom }}+A_{\text {Debye }}+A_{\mathrm{LD}}
$$

The magnitude of the LD force is given by the Hamaker constant. The Hamaker constant of the LD interaction has three components, shown in Eq. (7): a zero frequency term, a term that results from the vibrational contribution observed in the IR part of the frequency spectrum, and the electronic contribution observed in the UV part of the frequency spectrum.

$$
A_{\mathrm{LD}} \approx A_{\text {zero frequency }}+A_{\mathrm{IR} \text { (vibrational) }}+A_{\mathrm{UV} \text { (electronic) }}
$$

Bergström ${ }^{30}$ has estimated the magnitude of the IR contribution to the Hamaker constant. The IR contributions are important in systems, such as $\mathrm{BaTiO}_{3}$, that have strong vibrational modes. Water can also exhibit strong low-frequency excitations and, therefore, in, for example, biological lipid-water systems, lowfrequency contributions to the nondispersion forces and their temperature dependence can be prominent. ${ }^{44}$ In most other ceramic systems, the IR contribution is very small.

\section{(2) Lifshitz Theory and Electronic Structure and Bonding}

(A) Casimir Force: In 1948, Casimir ${ }^{16}$ used the recent developments in quantum electrodynamics (QED) to calculate the "attraction of two perfectly conducting plates" separated by vacuum. As a step beyond the simple pairwise additive summation of dipole/dipole interactions, Casimir was the first to use the fact that the optical properties of the perfectly conducting metallic plates are a direct measure of the virtual photon production and the intrinsic polarizability of the metal plates. This coupling of the fundamental optical properties to a direct calculation of the LD force between two bodies was a striking advance in the research. The attractive force he calculated for two metallic plates came to be known as the Casimir force. The existence of this theoretically predicted force stood unconfirmed until the recent work of Lamoreaux, ${ }^{18}$ who was able to directly measure it.

(B) Lifshitz Theory: In 1956, Lifshitz ${ }^{19}$ produced a general theory of the LD interaction for two solid materials separated by vacuum, basing the results on QED and quantum field theory. Lifshitz brought the connection between the fluctuating electric dipoles and the dielectric properties and electronic bonding of materials to the forefront. The derivation was formulated directly in terms of the dielectric and optical properties of the media involved. For two materials separated by vacuum, Lifshitz found the interaction obeys pairwise additivity and is always attractive.

(C) Repulsive Forces and the Assumption of Pairwise Additivity: In 1960, Dzyaloshinskii, Lifshitz, and Pitaevskii ${ }^{20}$ published another paper on two materials separated by a liquid film. The interlayer film, because of its own dielectric and optical properties, is also involved in the LD interaction, and it demonstrates the need for the many-body nature of the dispersion interaction to be considered. When an interlayer material is present, it effects the virtual photon exchange and the electromagnetic waves that induce the dipolar attraction in the two materials; the optical properties of the interlayer material 2 must be considered on an equal basis with the optical properties of materials 1 and 3. What we find is that the optical properties of the interlayer material (material 2) serve to renormalize the optical properties of the two ceramic grains or particles (materials 1 and 3 ). The original assumption of Hamaker, that the fundamental LD interaction is only pairwise in its nature, is incorrect; the interaction is fundamentally a many-body interaction.

The many-body nature of the LD interaction can radically change the dispersion force. Let us consider grains of $\mathrm{Al}_{2} \mathrm{O}_{3}$ with interlayers or coatings of $\mathrm{SiO}_{2}$ or air. We use the index of refraction, $n$, as a simple measure of the strength of the dispersion; $\mathrm{Al}_{2} \mathrm{O}_{3}, \mathrm{SiO}_{2}$, and air have $n$ values of $1.77,1.5$, and 1.0 , respectively. Consider the case shown in Fig. 2(a), where two grains of $\mathrm{Al}_{2} \mathrm{O}_{3}$ are separated by air. The Hamaker constant for this configuration is 165 zeptojoules $\left(1 \mathrm{zJ}=10^{-21} \mathrm{~J}\right)$, corresponding to an attractive intergranular force. Because the air interlayer can be considered equivalent to a vacuum interlayer, this LD interaction is pairwise in its nature, where only the bonds in the two grains need to be considered. However, if we change the air interlayer to a $\mathrm{SiO}_{2}$ interlayer, as shown in Fig. 2(b), then the Hamaker constant decreases to a value of $26.2 \mathrm{zJ}$, a dramatic reduction in the $\mathrm{LD}$ interaction. With the $\mathrm{SiO}_{2}$ interlayer, the interaction is no longer pairwise, and the interatomic bonds in the $\mathrm{SiO}_{2}$ serve to moderate the interaction of the bonds (or induced dipoles) in the two $\mathrm{Al}_{2} \mathrm{O}_{3}$ grains. The $n$ value of $\mathrm{SiO}_{2}$ produces a change in the wavelength of the virtual photons propagating between the $\mathrm{Al}_{2} \mathrm{O}_{3}$ grains. In this situation, the virtual photons that mediate the interaction actually have a longer optical path length as they travel between the $\mathrm{Al}_{2} \mathrm{O}_{3}$ grains. The higher value of $n$ of the interlayer material in Fig. 2(b) compared with Fig. 2(a) effectively renormalizes the separation between the grains, such that the $\mathrm{Al}_{2} \mathrm{O}_{3}$ grains can be considered to be at a greater physical separation, and the attractive dispersion force between the grains is reduced. The Hamaker constant is much decreased for the $\mathrm{SiO}_{2}$ interlayer.

If the two grains are of dissimilar materials, then the nonpairwise additivity of the LD interaction can change the forces from being only attractive to being repulsive. This is shown in Fig. 3(a), where an $\mathrm{Al}_{2} \mathrm{O}_{3}$ grain is separated by a $\mathrm{SiO}_{2}$ interlayer from a $\mathrm{MgF}_{2}$ grain (these materials have $n=1.77,1.5$, and 1.39, respectively). When $n$ decreases from material 1 to material 2 to material 3, then the Hamaker constant is negative, $-5.1 \mathrm{zJ}$ in this case, and corresponds to a repulsive LD force between the $\mathrm{Al}_{2} \mathrm{O}_{3}$ and the $\mathrm{MgF}_{2}$ grains. If we change the $\mathrm{MgF}_{2}$ to air, so that the configuration, as shown in Fig. 3(b), corresponds to an $\mathrm{Al}_{2} \mathrm{O}_{3}$ grain with a surface layer of $\mathrm{SiO}_{2}$, then we have the condition where the $n$ decreases from $\mathrm{Al}_{2} \mathrm{O}_{3}(n=1.77)$ to $\mathrm{SiO}_{2}(n=1.5)$ to air $(n=$ 1.0), the Hamaker constant is $-40.4 \mathrm{zJ}$, and the LD force is again repulsive. To understand this repulsive force, consider that it causes the air- $\mathrm{SiO}_{2}$ interface to be repelled from the $\mathrm{Al}_{2} \mathrm{O}_{3}-\mathrm{SiO}_{2}$ interface; the two interfaces of the $\mathrm{SiO}_{2}$ interlayer are repelled from each other. Thermodynamically, this corresponds to a wetting condition, where the dispersion interaction causing this repulsive force would produce a thickening of the $\mathrm{SiO}_{2}$ interlayer; were equilibrium achieved, the $\mathrm{SiO}_{2}$ interlayer would be of infinite thickness.

(D) LD and Interatomic Bonds: In 1961, Lifshitz produced a general theory for the LD forces. ${ }^{21,45,46}$ This formulation shows
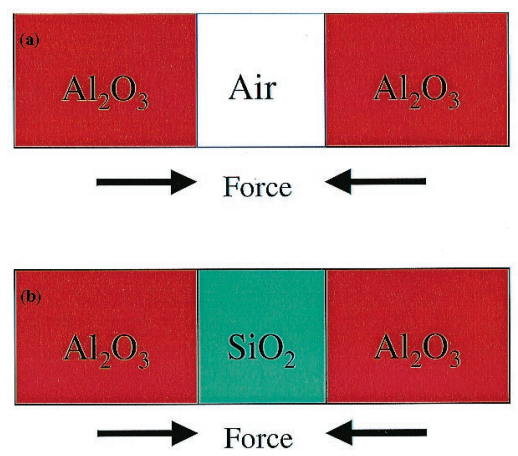

Fig. 2. Two configurations and their corresponding attractive LD forces: (a) two grains of $\mathrm{Al}_{2} \mathrm{O}_{3}$ separated by air, $A_{121}^{\mathrm{NR}}=165 \mathrm{zJ}$, and (b) two grains of $\mathrm{Al}_{2} \mathrm{O}_{3}$ separated by $\mathrm{SiO}_{2}, A_{121}^{\mathrm{NR}}=26.2 \mathrm{zJ}$. 

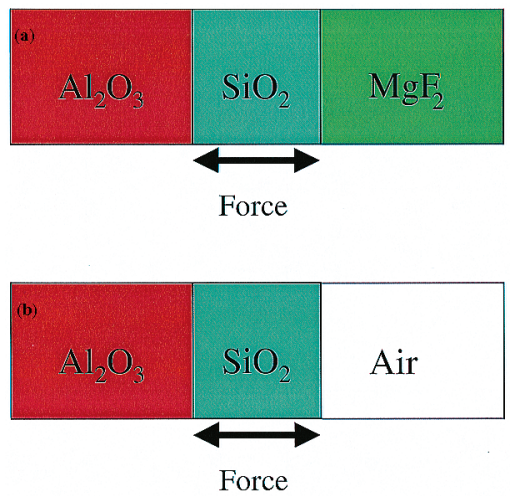

Fig. 3. Two configurations and their corresponding repulsive $\mathrm{LD}$ forces: (a) grain of $\mathrm{Al}_{2} \mathrm{O}_{3}$ and grain of $\mathrm{MgF}_{2}$ separated by an interlayer of $\mathrm{SiO}_{2}$ glass, $A_{123}^{\mathrm{NR}}=-5.1 \mathrm{zJ}$, and (b) $\mathrm{Al}_{2} \mathrm{O}_{3}$ grain coated by a layer of $\mathrm{SiO}_{2}$ glass, $A_{123}^{\mathrm{NR}}=-40.4 \mathrm{zJ}$.

that the most effective induced dipoles have frequencies in the visible through vacuum ultraviolet (VUV) spectral ranges. These induced dipoles can be interpreted in terms of the underlying electronic structure of the materials, as resulting from the interatomic bonds. To understand the electronic basis of the LD forces, consider the schematic model of two grains separated by a vacuum interlayer, as shown in Fig. 4, with $s$ and $p$ electronic orbitals in each material. Each of these bonds has a characteristic optical transition energy $E_{i}$ that corresponds to a characteristic frequency $\omega_{i}$ and wavelength $\lambda_{i}$, as given by Eq. (8), where $\hbar=h / 2 \pi$ is Planck's constant. For the induced dipole interactions to develop, the characteristic frequency of the dipoles in each grain must be comparable. Therefore, only the bonds at equivalent frequencies in each material interact, and the LD force results from the summation of the contributions of each interatomic bond that can find a corresponding bond of comparable frequency in the adjacent grain. Because of the requirement for comparable frequencies and energies for a dispersion interaction to develop, we see that the LD interaction is inherently based on spectral properties of the interacting materials and that these spectral properties correspond to the optical properties of the materials.

$$
E=\hbar \omega=\frac{h c}{\lambda}
$$

(E) Retardation of the LD Interaction at Long Distances: Lifshitz also introduced the concept that retardation of the LD interaction occurs when the interparticle spacing $\mathrm{L}$ becomes large in comparison to the transit time for the virtual photon across the interparticle gap. The Lifshitz theory includes the complete effects

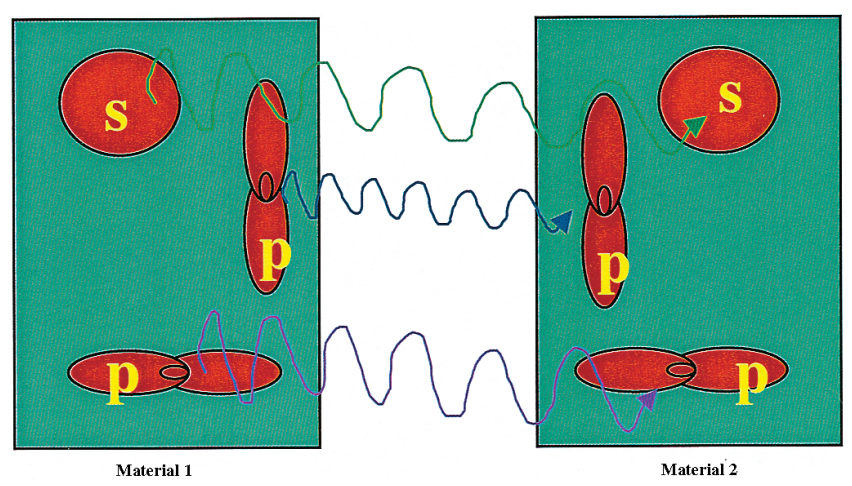

Fig. 4. Schematic model of two plane-parallel materials interacting across a gap. Three electronic interatomic bonds are shown with differing transition energies and frequencies in each grain. Interactions that develop between bonds of comparable frequencies contribute to the LD interaction. of retardation, of which the nonretarded case is one limit. Calculation of retarded dispersion forces is discussed in Section II(5) and applications are discussed in Section III(8).

Two limiting cases of the Hamaker constant are generally considered: nonretarded and retarded. When retardation of the dispersion interaction dominates, then, to first approximation, the power-law dependence of the dispersion interaction decreases, as given in Eqs. (9) and (10) for point-to-point interaction. This corresponds to a decrease in the dispersion force at large separation. Casimir and Polder, ${ }^{47}$ Lifshitz, and, more recently, Wennerström et al. ${ }^{48}$ have discussed the origins and effects of retardation, and Overbeek and co-workers ${ }^{49}$ in the 1950 s measured these forces, because they could not bring two solids to close enough approach to avoid the effects of retardation.

$$
\begin{aligned}
& E_{\text {disp }}^{\text {nonret }} \propto \frac{1}{L^{6}} \\
& E_{\text {disp }}^{\text {ret }} \propto \frac{1}{L^{7}}
\end{aligned}
$$

Retardation effects on the LD force do more than produce an accelerated decrease in the power law, as suggested by Eq. (10). The details of the electromagnetic interaction and the electronic structure of particular materials can produce new phenomena that are beyond the predictions of dispersion theory in the nonretarded regime. For example, dispersion forces can, without the effects of other interactions, produce surficial films that exhibit equilibrium thicknesses. This occurs in the case of the surface melting of ice ${ }^{50,51}$ whereby ice exhibits an equilibrium thickness film of liquid-water on its surface at temperatures below the freezing point, which results directly from the electronic structure and interatomic bonding of ice and liquid-water. This surface melting also is critical in ice skating, frost heaves, and lightning. ${ }^{52}$

$(F)$ Interband Transitions of the Electronic Structure and Optical Properties: (a) Electronic Structure: The electronic structure of materials represents the interatomic ionic, covalent, and metallic bonds formed by electrons that form the solid. ${ }^{53,54}$ The electronic structure can be considered from two general perspectives: that which provides information on the density of states (DOS) of the valence and conduction bands and that which supplies information on the joint density of states (JDOS) for electronic transitions from the occupied valence bands to the unoccupied conduction bands. ${ }^{55}$ Experimental techniques that produce information on the DOS are X-ray and UV photoelectron spectroscopy (XPS and UPS), inverse photoemission spectroscopy, and energy-loss near-edge structure (ELNES). The DOS also can be determined from first principles, using band structure techniques typically under the local density approximation, where the complete band diagram of the occupied and unoccupied electronic states is produced, and the density of these states per unit energy interval is determined. ${ }^{56,57}$

The LD interaction is an electromagnetic interaction, governed by Maxwell's equations, and, therefore, the JDOS perspective is the essential approach that couples the electronic structure and the dispersion forces. Consider the optical, interband transitions in the electronic structure, where the occupied valence band states are the initial states, and the unoccupied conduction band states are the final states. The JDOS corresponds to the matrix element for optical transitions between the valence and conduction band states. The matrix element is large for allowed transitions between bands with a large DOS at the transition energy and is smaller if the relative DOS of the two bands is less. The JDOS is zero if the transitions are not allowed or if no initial or final states are present at the transition energy. Because the JDOS includes the optical matrix element, it corresponds to the optical properties of the material and to optical transitions interband, i.e., from occupied to unoccupied states. The optical properties can be determined from first-principles band structure calculations, if, after completing the band structure calculation, the optical matrix elements are then calculated for all possible interband transitions, and these are then summed so as to produce the JDOS or a related optical property, 
such as the imaginary part of the dielectric constant, $\varepsilon_{2}$. The optical properties also can be determined from analysis of experimental optical data, such as reflectivity or energy loss. This is discussed in Section $\mathrm{II}(2)(\mathrm{F})(\mathrm{b})$.

It is the connection between the electronic structure of the materials and their optical properties that gives us both components of the LD interaction. The interatomic bonds in, e.g., material 1, as represented by the material's optical properties, produce the electromagnetic fluctuations that launch the virtual photons. These photons then interact with the interlayer materials (material 2) and the other grains (material 3) through Maxwell's equations and the optical properties of these other materials.

(b) Optical Properties: The propagation of electromagnetic waves in materials is governed by Maxwell's equations, and the optical properties of solids are the fundamental materials input. ${ }^{58}$ These optical properties are complex quantities in which the real and imaginary parts correspond to absorption and dispersion of light in the material. The dispersion of light is the change of the wavelength and speed of propagating light under the influence of the real part of the index of refraction of a material. ${ }^{14}$ There are algebraic relations among the numerous optical properties, such as the index of refraction $(\hat{n}=n+\mathrm{i} k)$, dielectric constant $\left(\hat{\varepsilon}=\varepsilon_{1}+\right.$ $\left.\mathrm{i} \varepsilon_{2}\right)$, optical conductivity $\left(\hat{\sigma}=\sigma_{1}+\mathrm{i} \sigma_{2}\right)$, and interband transition strength $\left(\hat{J}=J_{\mathrm{cv}, 1}+\mathrm{i} J_{\mathrm{cv}, 2}\right)$. Moreover, the Kramers-Kronig $(\mathrm{KK})$ dispersion relations govern the relationship of the real and imaginary components of any one optical property. Here we use the form presented by Wooten ${ }^{58}$ for optical property relations, and NIST $^{59}$ for fundamental physical constants.

Using VUV spectroscopy to measure optical reflectivity over a wide energy range, we can determine the optical properties of materials. (For more information on the experimental details of VUV and optical reflectivity and spectroscopic ellipsometry, see French and co-workers. ${ }^{60-65}$ The KK dispersion relations ${ }^{66-68}$ permit the analysis of reflectance, or other optical data, to obtain any conjugate pair of complex optical properties. The experimental data must span an infinite energy range and, because, in practice, experimental data are of limited range, these must be extended by extrapolation. For the optical reflectance, $R(E)$, and the reflected amplitude, $\rho(E)=R(E)^{1 / 2}$, the KK transform for reflectance ${ }^{69}(\mathrm{Eq}$. (11)) recovers the phase $\theta(E)$ of the reflected wave.

$$
\theta(E)=-\frac{2 E}{\pi} P \int_{0}^{\infty} \frac{\ln \rho\left(E^{\prime}\right)}{E^{\prime 2}-E^{2}} \mathrm{~d} E^{\prime}
$$

From the reflected amplitude and phase, any conjugate pair of optical properties can be computed. The complex index of refraction, for example, is obtained by solving Eq. (12), and the dielectric function is obtained by Eq. (13).

$$
\begin{aligned}
& \frac{n-1+\mathrm{i} k}{n+1+\mathrm{i} k}=\rho(E) e^{\mathrm{i} \theta(E)} \\
& \varepsilon_{1}+\mathrm{i} \varepsilon_{2}=(n+\mathrm{i} k)^{2}
\end{aligned}
$$

We typically render the optical response in terms of the interband transition strength, $J_{\mathrm{cv}}(E)$, which is related to $\varepsilon(\omega)$ by Eq. (14),

$$
J_{\mathrm{cv}}(E)=\frac{m_{\mathrm{o}}^{2}}{e^{2} \hbar^{2}} \frac{E^{2}}{8 \pi^{2}}\left(\varepsilon_{2}(E)+\mathrm{i} \varepsilon_{1}(E)\right)
$$

where $J_{\mathrm{cv}}(E)$ is proportional to the interband transition probability and has units of $\mathrm{g} \cdot \mathrm{cm}^{-3}$. For computational convenience, we take the prefactor $m_{\mathrm{o}}^{2} e^{-2} \hbar^{-2}$ in Eq. (14), whose value in cgs units is $8.289 \times 10^{-6} \mathrm{~g} \cdot \mathrm{cm}^{-3} \cdot \mathrm{eV}^{-2}$, as unity. Therefore, the units of the $J_{\text {cv }}(E)$ spectra are $\mathrm{eV}^{2}$. The interband transition strength of $\mathrm{Al}_{2} \mathrm{O}_{3}$ and AlN, as determined from VUV spectroscopy, are shown in Fig. 5. The differing energies and transition frequencies of the interatomic bonds in these two materials are visible in these spectra.

The oscillator strength sum rule, ${ }^{70,71}$ given in Eq. (15), for the

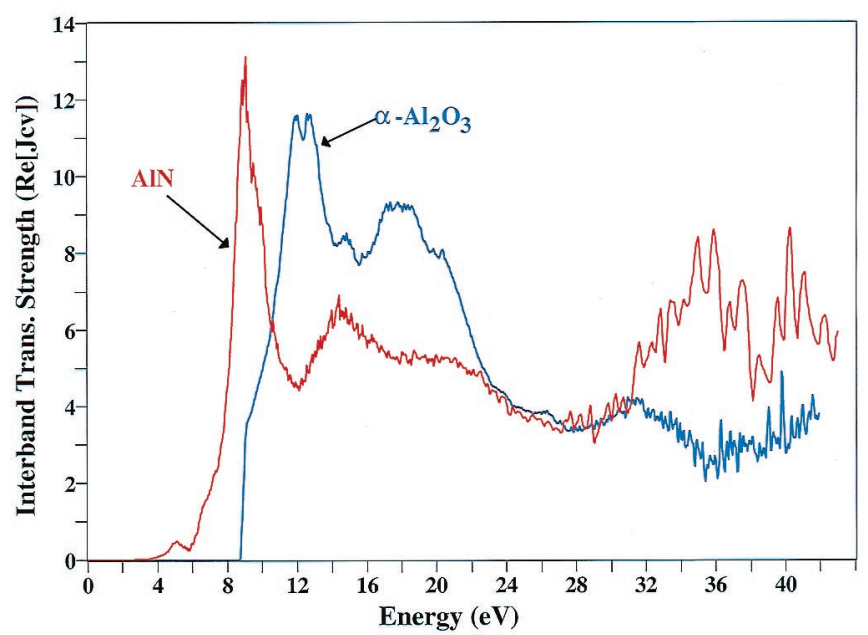

Fig. 5. Interband transition strengths, $J_{\text {cv }}$, of (red) $\mathrm{AlN}$ and $\mathrm{Al}_{2} \mathrm{O}_{3}$ (blue) determined from VUV reflectivity.

interband transition strength, $n_{\mathrm{eff}}(E)$, is

$$
n_{\mathrm{eff}}(E)=\frac{4 v_{f}}{m_{\mathrm{o}}} \int_{0}^{E} \frac{J_{\mathrm{cv}}\left(E^{\prime}\right)}{E^{\prime}} \mathrm{d} E^{\prime}
$$

where $v_{\mathrm{f}}$ is the volume of the formula unit $\left(20.86 \AA^{3}\right.$ for AIN and $4.25 \AA^{3}$ for $\mathrm{Al}_{2} \mathrm{O}_{3}$ ) gives the number of electrons contributing to the optical transitions up to an energy $E$. The results for AlN and $\mathrm{Al}_{2} \mathrm{O}_{3}$ are shown in Fig. 6.

Electron energy-loss spectroscopy (EELS) is a second experimental method that determines the energy-loss function $(\mathrm{ELF}=$ $-\operatorname{Im}(1 / \varepsilon)$ ), another of the optical properties of materials. EELS spectroscopy can be performed as a bulk or surface measurement in dedicated EELS spectrometers, but the spatially resolved (SR) EELS that is now common in electron microscopes has particular utility in the study of the LD forces. ${ }^{72}$ SR-EELS can be used to measure the ELF in the core EELS region (energy range from 200 to $>1000 \mathrm{eV}$ ), used for "fingerprinting" chemical constituents in materials; in the ELNES region (energy range from 70 to $500 \mathrm{eV}$ ), used for the conduction band DOS; and in the valence EELS (VEELS) region (energy range from 0 to $50 \mathrm{eV}$ ), used for the valence to conduction band transitions of the interband electronic structure. VEELS combined with the high spatial resolution available in the electron microscope (e.g., a scanning transmission electron microscope typically has a $0.5 \mathrm{~nm}$ probe diameter) is an exceptional probe of the electronic structure of interlayer materials and grains in ceramic systems.

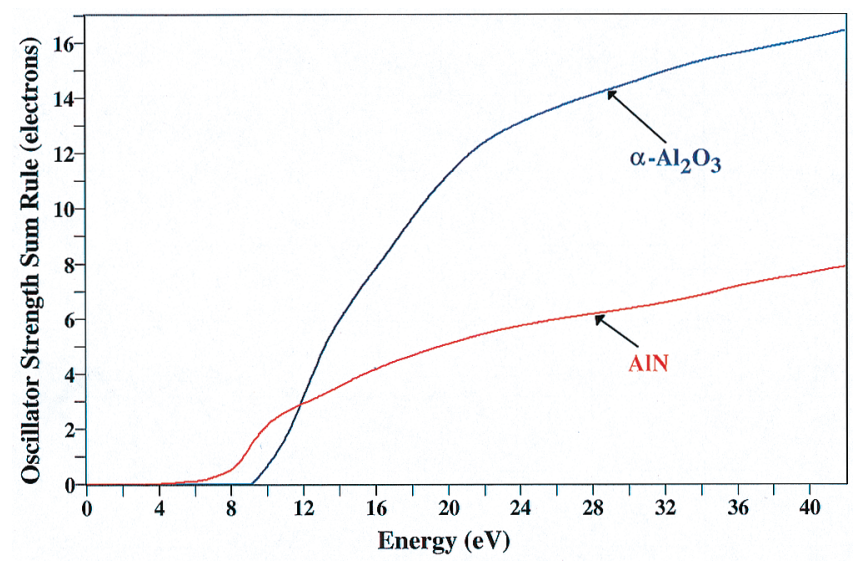

Fig. 6. Oscillator strength sum rule per formula unit of (red) AlN and (blue) $\mathrm{Al}_{2} \mathrm{O}_{3}$ determined from VUV reflectivity, showing the number of electrons involved in the optical transitions. 
The methods of VEELS have been discussed in detail. ${ }^{24,73,74}$ The experimentally determined ELF is first corrected for multiple scattering effects using Fourier logarithm deconvolution, then the ELF is scaled through application of the index sum rule shown in Eq. (16), where $P$ represents the Cauchy principal part of the integral.

$$
1-\frac{1}{n^{2}}=\frac{2}{\pi} P \int_{0}^{\infty} \operatorname{Im}\left(\frac{-1}{\varepsilon\left(E^{\prime}\right)}\right) \frac{1}{E} \mathrm{~d}\left(E^{\prime}\right)
$$

Once a single-scattering ELF with a quantitative amplitude has been determined, then the KK relation shown in Eq. (17) is used to determine $\operatorname{Re}(1 / \varepsilon)$ from the experimentally determined $-\operatorname{Im}(1 / \varepsilon)$.

$$
\operatorname{Re}\left(\frac{1}{\varepsilon(E)}\right)=1-\frac{2}{\pi} P \int_{0}^{\infty} \operatorname{Im}\left(\frac{-1}{\varepsilon\left(E^{\prime}\right)}\right) \frac{E^{\prime}}{E^{\prime 2}-E^{2}} \mathrm{~d}\left(E^{\prime}\right)
$$

From these results, any of the complex optical properties can be determined. The interband transition strength up to energies of 120 $\mathrm{eV}$ for a variety of ceramic materials is shown in Figs. 7 and $8 .{ }^{74}$ These spectra emphasize the very large differences in the interband electronic structure and optical properties of materials.

(G) LD Spectrum $\varepsilon_{2}(\xi)$ : The major work involved in calculations of Hamaker constants lies in determining or approximating the LD spectra $\varepsilon_{2}(\xi)$ for the materials of interest. Once the LD spectra have been determined, the particular values of the retarded or nonretarded Hamaker constant $A$ for a particular configuration of grains and interlayers constants is determined by computing integrals of the spectral difference functions $G$, which are discussed below.

When direct experimental data or first-principles calculations ${ }^{75}$ on the interband optical properties of the desired materials are available, we can use the KK relation given in Eq. (18) to determine the LD spectra following the method of Hough and White $^{76}$. The LD spectrum, $\varepsilon_{2}(\xi)$, is an integral transform of the imaginary part of the dielectric constant from a function of the real frequency $\omega$ to a function of the imaginary frequency $\xi .^{\dagger}$ The LD spectrum is an optical property and represents the retardation of the oscillators.

$$
\varepsilon_{2}(\xi)=1+\frac{2}{\pi} \int_{0}^{\infty} \frac{\omega \varepsilon_{2}(\omega)}{\omega^{2}+\xi^{2}} \mathrm{~d} \omega
$$

(H) Hamaker Constant $A^{\mathrm{NR}}$ : Once the LD spectra have been determined, then the Hamaker constant for different configurations

For the case of a simple oscillator or sums thereof, LD transform of $\varepsilon_{2}(\omega)$ to $\varepsilon_{2}(\xi)$ can be accomplished by the simple variable substitution of $i \xi$ for $\omega$ in the oscillator equation.

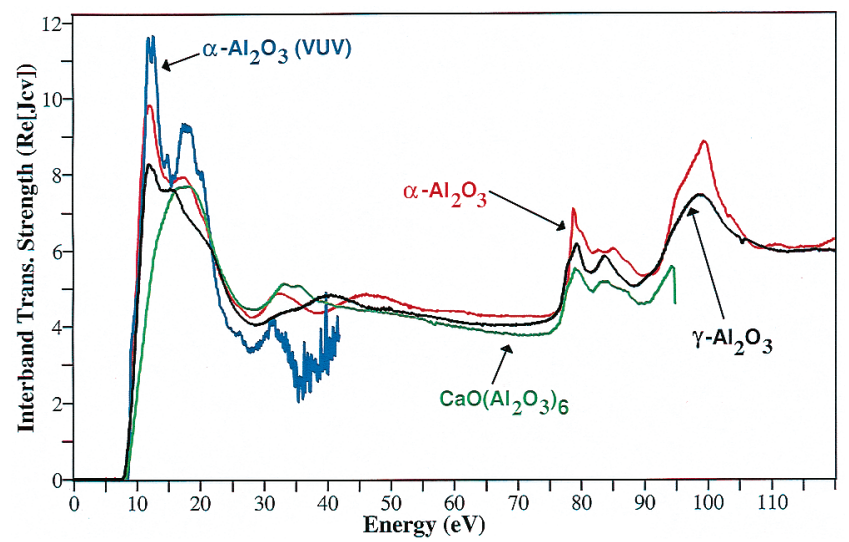

Fig. 7. Interband transition strengths, $J_{\mathrm{cv}}$, of three phases of $\mathrm{Al}_{2} \mathrm{O}_{3}$ determined from V-EELS with comparison to VUV results for $\alpha-\mathrm{Al}_{2} \mathrm{O}_{3}$. can be calculated. Following Lifshitz ${ }^{19}$ and Ninham and Parsegian, ${ }^{42}$ the interaction free energy per unit area resulting from the $\mathrm{LD}$ interaction can be given for one or more parallel films intervening between two media within a planar gap (see Fig. 1(a)) by Eq. (19), where $\xi$ is defined in Eq. (20).

$$
\begin{aligned}
& E=\frac{k T}{2 \pi} \sum_{n=0}^{\infty} \int_{0}^{\infty} \rho \mathrm{d} \rho \ln G\left(\xi_{n}\right) \\
& \xi_{n}=\frac{2 \pi k T}{\hbar} n
\end{aligned}
$$

At this point, we remark on the formalism of the complex frequency $\hat{\omega}$ of the oscillating dipoles defined in Eq. (21) (where $\left.\mathrm{i}=(-1)^{1 / 2}\right)$ :

$$
\hat{\omega}=\omega+i \xi
$$

From the interaction energy, we can, using Eq. (4), determine the Hamaker constant $A^{\mathrm{NR}}$.

$$
A^{\mathrm{NR}}=-6 k T L^{2} \sum_{n=0}^{\infty} \int_{0}^{\infty} \rho \mathrm{d} \rho \ln G\left(\xi_{n}\right)
$$

Now the configurations shown in Fig. 1 for the LD forces can be calculated. These are the nonretarded (denoted NR) Hamaker constants for three-layer geometries with a single film $\left(A_{121}^{\mathrm{NR}}, A_{123}^{\mathrm{NR}}\right)$ and five-layer geometries with a three-layer intervening film $\left(A_{12321}^{\mathrm{NR}}\right)$. These three types of Hamaker constants can be formulated on a common basis by defining three appropriate versions of the function $G(\xi)$ as follows:

$$
G_{121}^{\mathrm{NR}}(\xi)=1-\Delta_{12}^{2} e^{-2 a \rho}
$$

where $a$ is the thickness of the central layer (layer 2 for $\left(A_{121}^{\mathrm{NR}}\right.$, $A_{123}^{\mathrm{NR}}$ ), and layer 3 for $A_{12321}^{\mathrm{NR}}$ ),

$$
G_{123}^{\mathrm{NR}}(\xi)=1-\Delta_{32} \Delta_{12} e^{-2 a \rho}
$$

and

$$
G_{12321}^{\mathrm{NR}}(\xi)=1-\frac{\left(\Delta_{32}+\Delta_{21} e^{-2 b \rho}\right)^{2} e^{-2 a \rho}}{\left(1+\Delta_{32} \Delta_{21} e^{-2 b \rho}\right)^{2}}
$$

where $b$ is the invariant thickness of the intervening film between each particle and the central film in the case of $A_{12321}^{\mathrm{NR}}$ ) (Fig. 1). $\Delta$ is the difference of the LD spectra defined in Eq. (26).

$$
\Delta_{k j}=\frac{\varepsilon_{2, k}(\xi)-\varepsilon_{2, j}(\xi)}{\varepsilon_{2, k}(\xi)+\varepsilon_{2, j}(\xi)}
$$

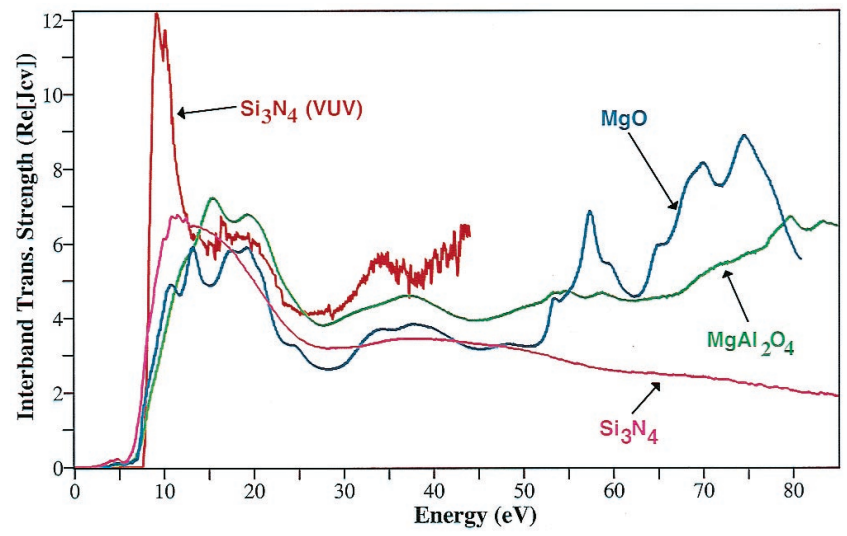

Fig. 8. Interband transition strengths, $J_{\text {cv }}$, of $\mathrm{MgO}, \mathrm{MgAl}_{2} \mathrm{O}_{4}$, and $\mathrm{Si}_{3} \mathrm{~N}_{4}$ determined from V-EELS with comparison to $\mathrm{Si}_{3} \mathrm{~N}_{4}$ determined from VUV spectroscopy. 
(3) Full Spectral Nonretarded Hamaker Constants $A^{\mathrm{NR}}$ from Spectral Optical Properties

With spectral optical properties over very wide energy ranges, as are available from optical reflectivity, VEELS measurements, or $a b$ initio band structure calculations, ${ }^{75}$ it is now possible to calculate Hamaker constants for the LD forces using the Lifshitz theory. We refer to Hamaker constants determined from this approach as full spectral (FS) Hamaker constants. The approach is summarized in Fig. 9. Once FS optical properties have been determined experimentally or from band structure calculations, the LD spectra are calculated and stored in a database of materials properties. These LD spectra are then used to calculate the spectral difference functions that are appropriate to the configuration of materials in the calculation of interest.

The details of the calculations of the FS Hamaker constants are given in Panel A.

(A) Estimated Corrections to FS Hamaker Constants $A^{\mathrm{NR}}$ : (a) Zero Frequency Term in the Lifshitz. Equation: The determination of Hamaker constants using the FS method contains the zero frequency term that results in the integral over frequency. This zero frequency term is weighted by one-half in the double summation form of the equations. In this presentation, we have explicitly shown the double summation form and the $3 k T / 2$ prefactor for clarity. The zero frequency term is correctly ${ }^{30}$ accounted for and evaluated in the FS Hamaker constant method. ${ }^{23}$

(b) Neglect of IR Vibrational Results in LD Spectra: In the calculation of the LD spectra, we use experimental optical properties that typically extend from a low energy of $2-4 \mathrm{eV}$ to a high energy of 40-140 eV. In this way we contain all the interband electronic transitions in the experimental data range. In materials that have large dielectric polarizabilities from IR vibrational modes, these modes produce an IR contribution to the optical properties that adds to the value of the Hamaker constant. Because of the form of the integrals, they are heavily weighted to the electronic contributions resulting in the UV and VUV optical ranges. However, in systems with very low optical contrast between the materials-i.e., very-well-matched optical properties or indexes of refraction for the grains and the interlayer-the Hamaker constant resulting from the UV-electronic contributions can be very small, e.g., $<1-5 \mathrm{zJ}$. When there is very low contrast, even a small contribution to the Hamaker constant from IR vibrations excitation may be decisive if the contribution is large enough. Bergström ${ }^{30}$ discussed this IR vibrational contribution, evaluating its magnitude for two cases: $\mathrm{BaTiO}_{3}$, which has a very high zero frequency dielectric constant resulting from IR vibrational modes, and $\mathrm{SiO}_{2}$, a material whose zero frequency dielectric constant is determined by its UV electronic transitions. Bergström found that, for $\mathrm{BaTiO}_{3}$, the IR vibrational contribution to the Hamaker constant is substantial, corresponding to an increase of the Hamaker constant by as much as $50 \mathrm{zJ}$ for interlayers from vacuum to hydrocarbon solvents. For a material such as $\mathrm{SiO}_{2}$, the IR vibrational contribution is negligible, corresponding to an increase of $1.5 \mathrm{zJ}$ for $A^{\mathrm{NR}}\left[\mathrm{SiO}_{2} \mid\right.$ vacuum $\left.\mid \mathrm{SiO}_{2}\right]$ to a value of 65



Fig. 9. Schematic diagram for the determination of full spectral Hamaker constants from various forms of spectral optical properties determined from experiment or theory.
zJ. In $\mathrm{SiO}_{2}$, the IR vibrational contribution is important only in the case where the UV electronic contribution to the Hamaker constant approaches $0 \mathrm{zJ}$. The $\mathrm{SiO}_{2}$ case is more characteristic for ceramic materials, where the IR vibrational contributions are negligible.

(c) Changes in the Spectral Optical Properties: The accuracy of the input optical properties and the resulting LD spectra are critical to the accuracy of the calculated FS Hamaker constants. Consider the case of $\mathrm{Al}_{2} \mathrm{O}_{3}$, in which the optical properties of $\mathrm{Al}_{2} \mathrm{O}_{3}$ published in $1998,{ }^{62,64}$ shown in Fig. 10, have improved accuracy to those published in $1994^{54}$ because of a more accurate implementation of the KK boundary conditions of the reflectance outside the experimental data range. The 1998 optical property values led to changes in the amplitude of the interband transition strength at higher energies. These refinements in optical properties are not unusual; The Handbook of Optical Constants of Solids ${ }^{77}$ publishes critiques of the literature values of the optical properties of materials, such as $\mathrm{Al}_{2} \mathrm{O}_{3},{ }^{78,79}$ and these demonstrate the consensus values of the optical properties or the disagreements. The very small changes from 1994 to 1998 correspond only to changes in the index of refraction, determined by the index sum rule: ${ }^{70} n\left(\mathrm{Al}_{2} \mathrm{O}_{3}-1994\right)=1.75$ and $n\left(\mathrm{Al}_{2} \mathrm{O}_{3}-1998\right)=1.77$.

Changes in the FS Hamaker constants of $\mathrm{Al}_{2} \mathrm{O}_{3}$ calculated using the 1998 and 1994 versions of the $J_{\text {cv }}$ spectra are shown in Table II. Examples are given for vacuum, water, and $\mathrm{SiO}_{2}$ interlayers and for a surficial $\mathrm{SiO}_{2}$ film on $\mathrm{Al}_{2} \mathrm{O}_{3}$. With the largest Hamaker constant (shown for the vacuum interlayer, because it has the largest index contrast with $\mathrm{Al}_{2} \mathrm{O}_{3}$ ), $A_{121}^{\mathrm{NR}}$ increases $20 \mathrm{zJ}$ from 145 to $165 \mathrm{zJ}$ with the 1998 spectra, corresponding to a $14 \%$ increase. For lower-contrast interlayers, such as $\mathrm{SiO}_{2}, A_{121}^{\mathrm{NR}}$ increases from 18 to $26 \mathrm{zJ}$, corresponding to a $45 \%$ increase.

The quantitative accuracy of the optical properties used in FS Hamaker constant calculations contributes the largest uncertainty in the magnitude of $A^{\mathrm{NR}}$. These uncertainties in the input optical properties also impact the simple spectral and index approximation Hamaker constant calculations discussed in Section II(4), because these methods are all based on fits to the fundamental optical properties.

The uncertainties in the absolute magnitude of $A^{\mathrm{NR}}$ due to the accuracy of input spectra do not impact comparisons of and trends

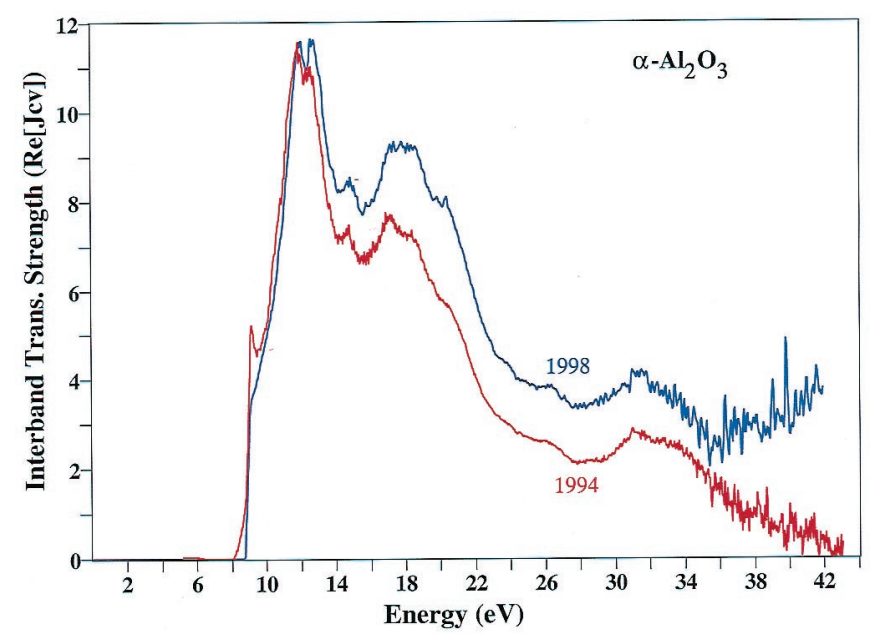

Fig. 10. Interband transition strength, $J_{\text {cv }}$, of $\mathrm{Al}_{2} \mathrm{O}_{3}$ published in (red) $1994^{54}$ and (blue) $1998^{62}$.

Table II. Variation in Hamaker Constant $A^{\text {NR }}$ because of Changes in Optical Properties Spectra

\begin{tabular}{|c|c|c|}
\hline & $\mathrm{Al}_{2} \mathrm{O}_{3}$ spectra 1994 & $\mathrm{Al}_{2} \mathrm{O}_{3}$ spectra 1998 \\
\hline \begin{tabular}{l|l|l|l}
$A^{\mathrm{NR}}\left[\mathrm{Al}_{2} \mathrm{O}_{3}\right.$ & vacuum $\left.\mid \mathrm{Al}_{2} \mathrm{O}_{3}\right]$ \\
$A^{\mathrm{NR}}\left[\mathrm{Al}_{2} \mathrm{O}_{3}\right.$ & water| & $\left.\mathrm{Al}_{2} \mathrm{O}_{3}\right]$ \\
$A^{\mathrm{NR}}\left[\mathrm{Al}_{2} \mathrm{O}_{3}\right.$ & $\mathrm{SiO}_{2}$ & $\left.\mathrm{Al}_{2} \mathrm{O}_{3}\right]$ \\
$A^{\mathrm{NR}}\left[\mathrm{Al}_{2} \mathrm{O}_{3}\right.$ & $\mathrm{SiO}_{2}$ & air $]$
\end{tabular} & $\begin{array}{r}145 \mathrm{zJ} \\
44.6 \mathrm{zJ} \\
17.6 \mathrm{zJ} \\
-33.9 \mathrm{zJ}\end{array}$ & $\begin{array}{r}164.9 \mathrm{zJ} \\
57.0 \mathrm{zJ} \\
26.2 \mathrm{zJ} \\
-40.4 \mathrm{zJ}\end{array}$ \\
\hline
\end{tabular}




\section{Panel A: Full Spectral Hamaker Constants $A^{\mathrm{NR}}$}

\section{(1) Calculation of $\varepsilon_{2}(\xi)$ Spectra}

Once the complex optical properties as a function of the real frequency $\omega$ have been determined, the LD integral transform (Eq. (18)) must be applied. The LD transform requires data over an infinite frequency or energy range, and, therefore, we use analytical extensions or wings to continue the data beyond the experimental data range. We choose power-law wings of the form $\operatorname{Re}\left[J_{\mathrm{cv}}\right] \propto \omega^{-\alpha}$ on the low-energy side of the data and $\operatorname{Re}\left[J_{\mathrm{cv}}\right] \propto \omega^{-\beta}$ on the high-energy side of the data. We have chosen fixed values of $\beta=$ 3 and $\alpha=2$. The wings are extended to cover from 0 to $100 \mathrm{eV}$ to minimize errors due to neglected areas in the ensuing integrals used in Hamaker constant determination. On determining the LD spectrum, we retain the complete spectrum over the entire $0-100$ $\mathrm{eV}$ range, not the limited experimental data range, to facilitate the evaluation of the spectral difference functions while continuing to minimize errors resulting from neglected areas between the $\varepsilon_{2}(\xi)$ spectra.

The interband transition strengths and LD spectra for $\mathrm{Si}_{3} \mathrm{~N}_{4}$ and an YAl-SiON glass ${ }^{23}$ are shown in Fig. A1.

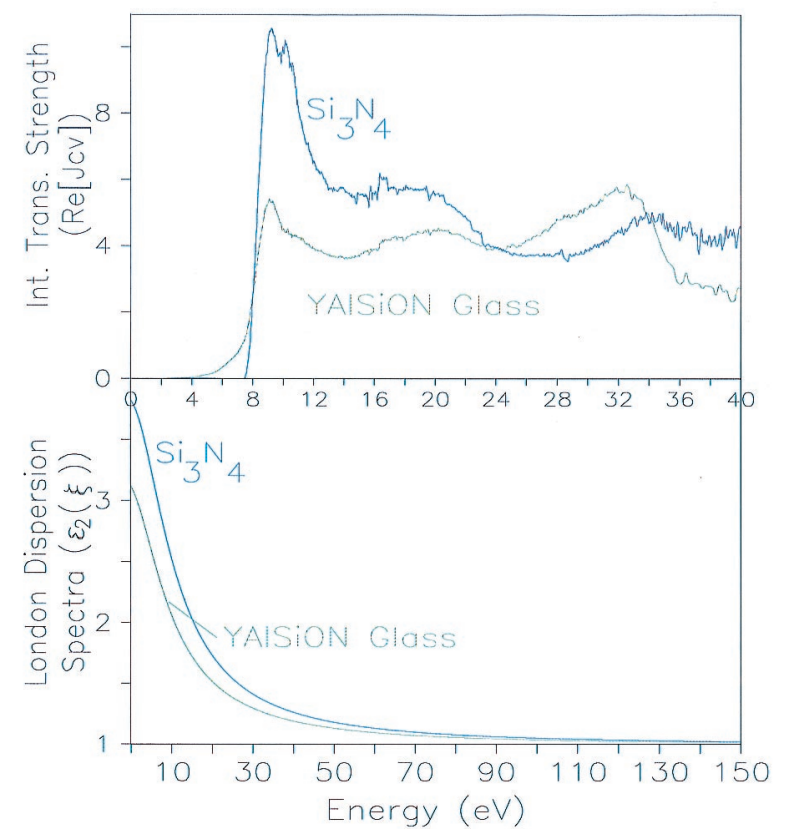

Fig. A1. Interband transition strengths and the corresponding LD spectra for $\mathrm{Si}_{3} \mathrm{~N}_{4}$ and an YAl-SiON glass. Hamaker constant for $A^{\mathrm{NR}}$ $\left[\mathrm{Si}_{3} \mathrm{~N}_{4} \mid\right.$ vacuum $\left.\mid \mathrm{Si}_{3} \mathrm{~N}_{4}\right]=192 \mathrm{zJ}$, whereas the Hamaker constant of YAl-SiON glass as the interlayer between the $\mathrm{Si}_{3} \mathrm{~N}_{4}$ grains, is $A^{\mathrm{NR}}$ $\left[\mathrm{Si}_{3} \mathrm{~N}_{4}|\mathrm{YAl}-\mathrm{SiON}| \mathrm{Si}_{3} \mathrm{~N}_{4}\right]=9.3 \mathrm{zJ}$. (Reprinted with permission from Elsevier Science. ${ }^{24}$ )

After the LD spectra $\varepsilon_{2}(\xi)$ are calculated, they are accumulated in a spectral database ${ }^{\ddagger}$ from which differing combinations can be used to calculate Hamaker constants. One of the properties of $\varepsilon_{2}(\xi)$ is that the value at zero energy is the square of the real part of the index of refraction. Therefore, we can check the validity of the $\varepsilon_{2}(\xi)$ analysis by comparing the implied index to the known index of the material in the visible.

\section{(2) Full Spectral Hamaker Constants: Calculation of $A^{N R}$ from London Dispersion Spectra}

Once we have determined the complex optical properties and calculated the LD spectra, we can calculate the Hamaker constants for many physical geometries. However, the integrals given in Eq. (22) of the $G$ functions of Eqs. (23)-(25) are not amenable to direct evaluation. We need to reformulate the integral over wave vector $(d \rho)$ into a summation and to change the infinite frequency sum into one over the finite data range available experimentally. Here we extend the formulation of Hough and White. ${ }^{76}$

(A) Integral over the Wave Vector $(\rho)$ : We simplify the Hamaker constant Eqs. (23)-(25) by first transforming the integral on the wave vector $\rho$ into a summation. The results of this transformation are given in Eqs. (27)-(29) for $A_{121}^{\mathrm{NR}}, A_{123}^{\mathrm{NR}}$, and $A_{12321}^{\mathrm{NR}}$, respectively.

$$
\begin{aligned}
& A_{121}^{\mathrm{NR}}=6 k T L^{2} \frac{1}{4 a^{2}} \sum_{n=0}^{\infty}\left(+\Delta_{12}^{2}+\frac{\Delta_{12}^{4}}{8}+\frac{\Delta_{12}^{6}}{27}+\ldots\right)=\frac{3 k T}{2} \sum_{n=0}^{\infty} \sum_{s=1}^{\infty} \frac{\left(\Delta_{12}^{2}\right)^{s}}{s^{3}} \\
& A_{123}^{\mathrm{NR}}=\frac{3 k T}{2} \sum_{n=0}^{\infty} \sum_{s=1}^{\infty} \frac{\left(\Delta_{32} \Delta_{12}\right)^{s}}{s^{3}} \\
& A_{12321}^{\mathrm{NR}}=\frac{3 k T}{2} \sum^{\prime}\left\{\sum_{s=1}^{\infty}\left[\frac{\Delta_{32}^{2 s}}{s^{3}}\right]+\sum_{s=1}^{\infty}\left[\frac{\Delta_{21}^{2 s}}{(1+2 b / a)^{2} s^{3}}\right]+\sum_{s=1}^{\infty}\left[\prod_{k=1}^{s} \frac{2(2 k-1)}{k} \frac{\Delta_{32}^{s} \Delta_{21}^{s}}{(1+b / a)^{2} s^{3}}\right]+\begin{array}{c}
\text { additional } \\
\text { cross } \\
\text { terms }
\end{array}\right\}
\end{aligned}
$$

GRAMS is a PC-based spectroscopy environment in which the ELECTRONIC STRUCTURE TOOLS (EST), and HAMAKER.AB, a component of EST, have been developed. GRAMS is available from Galactic Industries, Salem, NH (http://www.galactic.com). HAMAKER.AB and its spectral database of LD spectra is available as part of EST from Deconvolution and Entropy Consulting, Ithaca, NY (http://www.deconvolution.com). 


\section{Panel A: Continued}

The $A_{12321}^{\mathrm{NR}}$ equation can be used for values of $b$ such that the volume fraction $V_{\mathrm{f}}$ of material 2 in the film, defined as $V_{\mathrm{f}}=2 b / L$, varies from $0<V_{\mathrm{f}} \ll 1$, subject to the approximations that permit the expansions, e.g., $\Delta_{22} \Delta_{21} e^{-2 b \rho} \ll 1$.

(B) Summation over Frequency $(d \xi)$ : These integrals have been written for evaluation over an infinite imaginary frequency range in $\xi$; however, we have determined the LD spectra for a range of 0 to $100-150 \mathrm{eV}$. Moreover, the LD spectra have a sampling interval $\delta \xi$ that equals the experimental data-point spacing. Therefore, to cover the general case, where many spectra are of variable energy range and sampling density, we first select an upper energy limit for summation, $E_{\text {lim }}$, which is determined by the smallest energy range available, and then we resample the LD spectra at $0.054 \mathrm{eV}(T=100 \mathrm{~K})$. With this sampling interval, we are in a position to calculate Hamaker constants at any temperatures that are multiples of $100 \mathrm{~K}$. Therefore, the equations used herein to determine the Hamaker constants for the various cases become, in the explicit form of a double summation, as follows:

$$
\begin{aligned}
& A_{121}^{\mathrm{NR}}=\frac{3 k T}{2} \sum_{n=0}^{E_{\lim }}, \sum_{s=1}^{4} \frac{\left(\Delta_{12}^{2}\right)^{s}}{s^{3}} \\
& A_{123}^{\mathrm{NR}}=\frac{3 k T}{2} \sum_{E=0}^{E_{\lim }} \sum_{s=1}^{4} \frac{\left(\Delta_{32} \Delta_{12}\right)^{s}}{s^{3}} \\
& A_{12321}^{\mathrm{NR}}=\frac{3 \delta E}{4 \pi} \sum_{E=0}^{E_{\text {lim }}},\left\{\sum_{s=1}^{4}\left[\frac{\Delta_{32}^{2 s}}{s^{3}}\right]+\sum_{s=1}^{4}\left[\frac{\Delta_{21}^{2 s}}{(1+2 b / a)^{2} s^{3}}\right]+\sum_{s=1}^{4}\left[\prod_{k=1}^{s} \frac{2(2 k-1)}{k} \frac{\Delta_{32}^{s} \Delta_{21}^{s}}{(1+b / a)^{2} s^{3}}\right]+\begin{array}{c}
\text { additional } \\
\text { cross } \\
\text { terms }
\end{array}\right\}
\end{aligned}
$$

In these equations, the primed summation represents that only one-half of the first term in the frequency (energy) sum is counted, and the summation is understood to include only terms that satisfy the sampling interval $\delta E=2 \pi k T$. The temperature-dependent sampling interval results from the presence of $\operatorname{coth}(\hbar \omega / 2 k T)$ in Lifshitz's ${ }^{19}$ molecular force equation (Eq. (24)), which changes an integral over all frequencies to a sum. The one-half weight given to the zero energy term was a necessary step when the integral over all frequencies was changed to an integral over positive frequencies.

in Hamaker constants for a series of materials configurations. The variation of $A^{\mathrm{NR}}$ with differing interlayers shown in Table II is comparable for both of the $\mathrm{Al}_{2} \mathrm{O}_{3}$ spectra used.

(d) Neglect of (d $\xi)$ Frequency Integral from $100 \mathrm{eV}$ to Infinity: In the FS Hamaker constant method, we typically restrict the range of the LD spectra to an energy of $100 \mathrm{eV}$, whereas the integrals over frequency should extend to infinity. We neglect the contribution to the integrals or sums of the spectral difference functions, $\Delta_{k j}$, given by Eq. (26), from $100 \mathrm{eV}$ to infinity. We can estimate the "missing" contribution to the integrals of the LD spectra from $100 \mathrm{eV}$ to infinity by recognizing that, at high energies, $\Delta_{k j}$ is proportional to $E^{-2}$. At high energies, the summations on $s$ in Eqs. (30)-(32) are proportional to $E^{-4}$ plus other terms that decrease more rapidly with increasing energy. Therefore, to estimate this high-energy $(>100 \mathrm{eV})$ contribution to $A^{\mathrm{NR}}$, we fit an $E^{-4}$ wing to the energy spectrum and then analytically integrate this term. We calculate the high-energy contribution for $A_{121^{-}}^{\mathrm{NR}}$ and $A_{123^{\mathrm{NR}}}^{\mathrm{NR}}$-type Hamaker constants with high and low optical contrast between the grains and the interlayer materials, and we summarize these in Table III. These high-energy corrections to the Hamaker constant are $<1 \mathrm{zJ}$ in all cases and correspond to errors on the order of $0.1 \%-0.8 \%$. This estimate of the high-energy contribution is usually larger than the temperature dependence of the Hamaker constant from 0 to $300 \mathrm{~K}$ because of the $k T$ terms and sampling effect, which is discussed in Section $\mathrm{II}(3)(\mathrm{C})(\mathrm{e})$. (e) Temperature Dependence of Hamaker Constant $A^{\mathrm{NR}}$ : In the calculation of the Hamaker constant, the summation over frequency shown in Eqs. (30)-(32) requires a temperaturedependent sampling of the $\Delta_{k j}$ in units of $\delta E=2 \pi k T$, along with the explicit $k T$ prefactor. These two effects produce an explicit temperature dependence of the Hamaker constant. The temperature dependence due to the $k T$ prefactor and frequency sampling for the $A_{121}^{\mathrm{NR}}$ - and $A_{123}^{\mathrm{NR}}$-type Hamaker constants is presented in Table IV. Table IV shows that this temperature dependence is, in all cases, $<0.2 \mathrm{zJ}$ and corresponds to a contribution to the Hamaker constant of $<0.3 \%$ for a temperature range of $2000 \mathrm{~K}$.

In addition to the temperature dependence explicit in the $k T$ prefactor and the frequency sampling, a much larger contribution to the Hamaker constant results from the temperature dependence of the electronic structure and optical properties of materials. It has been shown ${ }^{53}$ that the band gap of $\alpha-\mathrm{Al}_{2} \mathrm{O}_{3}$ decreases with increasing temperature at the rate of $1.1 \mathrm{meV} / \mathrm{K}$. The index of refraction of $\mathrm{Al}_{2} \mathrm{O}_{3}$ as a function of temperature is shown in Fig. 11. Initially, the index increases, only to decrease for temperature above $\sim 1000 \mathrm{~K}$. The temperature dependence of the $A_{1 \mathrm{v} 1}^{\mathrm{NR}} \mathrm{Ha}-$ maker constant shows a similar increase and subsequent decrease at higher temperatures. This type of second-order variation in the Hamaker constant, resulting from the experimentally determined changes in the interband electronic structure, goes beyond any simple theories of the temperature dependence of the Hamaker

\begin{tabular}{|c|c|c|c|}
\hline Material 1 & $\mathrm{TiO}_{2}$ & $\mathrm{Al}_{2} \mathrm{O}_{3}$ & $\mathrm{SiO}_{2}$ \\
\hline
\end{tabular}
constant and LD force. Moreover, the changes in the Hamaker

Table III. Hamaker Constant $A^{\mathrm{NR}}$ and the Contribution to $A^{\mathrm{NR}}$ because of Frequency Summation from $100 \mathrm{eV}$ to Infinity 
Table IV. Temperature Dependence of the Hamaker Constant because the $k T$ Prefactor and Frequency Sampling Effects

\begin{tabular}{|c|c|c|c|}
\hline & $0 \mathrm{~K}$ & $300 \mathrm{~K}$ & $2000 \mathrm{~K}$ \\
\hline \begin{tabular}{l|l|l|l}
$A^{\mathrm{NR}}\left[\mathrm{Al}_{2} \mathrm{O}_{3}\right.$ & vacuum $\mid \mathrm{Al}_{2} \mathrm{O}$ \\
$A^{\mathrm{NR}}\left[\mathrm{Al}_{2} \mathrm{O}_{3}\right.$ & water & $\left.\mathrm{Al}_{2} \mathrm{O}_{3}\right]$ \\
$A^{\mathrm{NR}}\left[\mathrm{Al}_{2} \mathrm{O}_{3}\right.$ & $\mathrm{SiO}_{2}$ & $\left.\mathrm{Al}_{2} \mathrm{O}_{3}\right]$ \\
$A^{\mathrm{NR}}\left[\mathrm{Al}_{2} \mathrm{O}_{3}\right.$ & $\mathrm{SiO}_{2}$ & air $]$
\end{tabular} & $\begin{array}{l}164.85 \mathrm{zJ} \\
56.998 \mathrm{zJ} \\
26.1565 \mathrm{zJ} \\
-40.4044 \mathrm{zJ}\end{array}$ & $\begin{array}{r}164.851 \mathrm{zJ} \\
56.9786 \mathrm{zJ} \\
26.1568 \mathrm{zJ} \\
-40.4047 \mathrm{zJ}\end{array}$ & $\begin{array}{r}164.864 \mathrm{zJ} \\
56.8087 \mathrm{zJ} \\
26.1604 \mathrm{zJ} \\
-40.4081 \mathrm{zJ}\end{array}$ \\
\hline
\end{tabular}

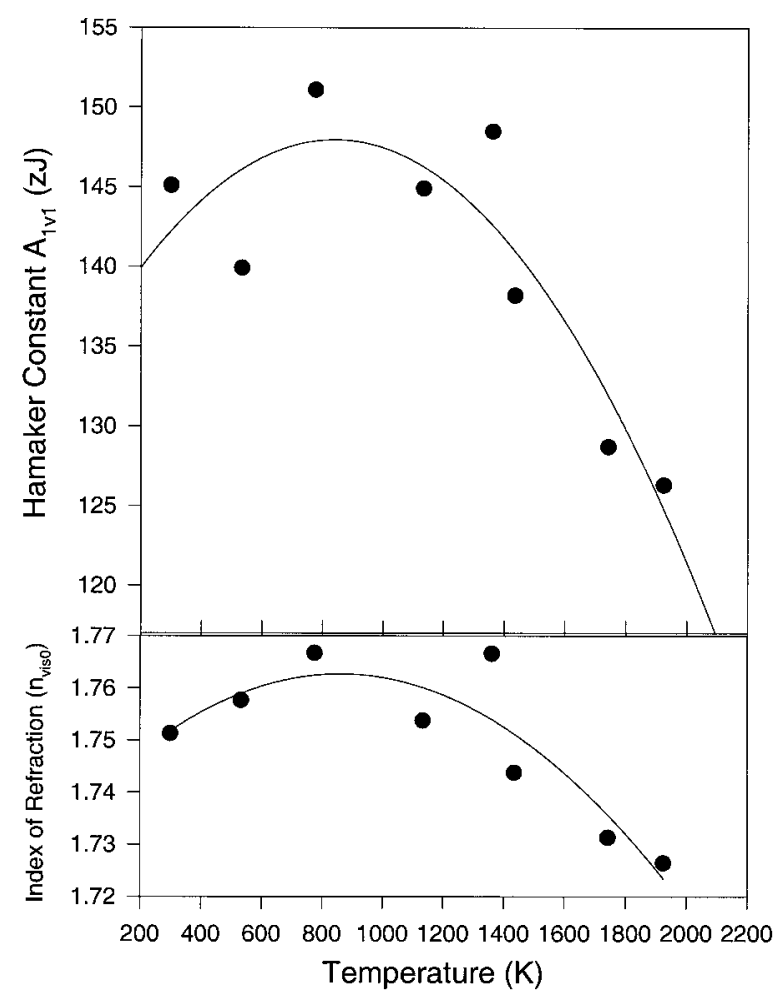

Fig. 11. Temperature dependence of the Hamaker constant $A_{1 \mathrm{v} 1}^{\mathrm{NR}}$ and the index of refraction for $\alpha-\mathrm{Al}_{2} \mathrm{O}_{3}$ up to $1925 \mathrm{~K}$. (Reprinted with permission from Elsevier Science. ${ }^{23}$ )

constant are large, varying from 140 to $148 \mathrm{zJ}$ and then decreasing to $125 \mathrm{zJ}$, which correspond to variations in the Hamaker constant of $6 \%-11 \%$.

\section{(4) Methods for Estimating Hamaker Constant $A^{\mathrm{NR}}$}

Where extensive experimental data are not yet available, simple spectral models or index approximations can be used to estimate the Hamaker constant for various configurations. Because Lifshitz theory for calculating $A$ is based on the use of optical properties as the fundamental input, various methods have been developed to estimate the optical properties for materials over the wide energy range needed. These methods ${ }^{25}$ can be divided into two general classes: the simple spectral methods, ${ }^{30,76}$ which use optical data in the visible to develop parametric Lorentz oscillator ${ }^{58}$ models of the LD spectra over a wide energy range, ${ }^{80}$ and index approximations, such as the Tabor Winterton approximation (TWA), which are based on the index of refraction as a measure of a material's dispersion.

(A) Simple Spectral Method: To calculate Hamaker constants based on the Lifshitz theory, Ninham and co-workers ${ }^{32,42}$ introduced the simple spectral (SS) method, ${ }^{30,76,81}$ which uses a set of parametric damped Lorentz oscillators to represent the LD spectrum. Essentially, these oscillators correspond to absorptions in the material at certain frequencies of given strengths, and these oscillators can be fitted to physical property data, such as absorption spectra, refractive index, or dielectric constant. A model LD spectra can be synthesized from the relation

\section{Panel B: Index Approximations of $A^{\mathrm{NR}}$}

\section{(1) Tabor Winterton Approximation}

Assuming the optical properties of the necessary materials can be described as resulting from a single LO and using some additional approximations, an analytic approximation for $A_{123}^{\mathrm{NR}}$, the TWA ${ }^{93}$ can be obtained as ${ }^{94}$

$$
\begin{aligned}
& A_{123}^{\mathrm{TWA}}=\frac{3 \pi \hbar v_{\mathrm{e}}}{4 \sqrt{2}}[\left.\left(n_{\mathrm{vis} 0,1}^{2}-n_{\mathrm{vis} 0,2}^{2}\right)\left(n_{\mathrm{vis} 0,3}^{2}-n_{\mathrm{vis} 0,2}^{2}\right)\right] \\
& /\left\{\left(n_{\mathrm{vis} 0,1}^{2}+n_{\mathrm{vis} 0,2}^{2}\right)^{1 / 2}\left(n_{\mathrm{vis} 0,3}^{2}+n_{\mathrm{vis} 0,2}^{2}\right)^{1 / 2}\right. \\
&\left.\quad \times\left[\left(n_{\mathrm{vis} 0,1}^{2}+n_{\mathrm{vis} 0,2}^{2}\right)^{1 / 2}+\left(n_{\mathrm{vis} 0,3}^{2}+n_{\mathrm{vis} 0,2}^{2}\right)^{1 / 2}\right]\right\}
\end{aligned}
$$

which depends on the limiting values of the index of refraction in the visible $\left(n_{\text {vis }}\right)$, extrapolated to zero energy $\left(n_{\mathrm{vis} O}\right)$, for the $i$ materials $\left(n_{\mathrm{vis}, i}\right)$, and the characteristic absorption frequency $\left(v_{\mathrm{e}}\right)$, which is related to the bandgap and is assumed to be equivalent for all three materials so as to permit the integrations. When materials 1 and 3 are identical, the TWA reduces to

$$
A_{121}^{\mathrm{TWA}}=\frac{3 \pi \hbar \nu_{\mathrm{e}}}{8 \sqrt{2}} \frac{\left(n_{\mathrm{vis} 0,1}^{2}-n_{\mathrm{vis} 0,2}^{2}\right)^{2}}{\left(n_{\mathrm{vis} 0,1}^{2}+n_{\mathrm{vis} 0,2}^{2}\right)^{3 / 2}}
$$

The TWA is predicated on the assumption that the predominant contribution to the LD forces results from interband transitions in or near the visible and UV range associated with the primary bandgap of the material. Typically, a characteristic absorption frequency of $v_{\mathrm{e}}=3 \times 10^{15} \mathrm{~Hz}$ is used in TWA calculations

\section{(2) Single Oscillator Approximation}

The single oscillator approximation $(\mathrm{SOA})^{87}$ (Eq. (38)) is another Hamaker constant approximation similar to the TWA. However, instead of assuming the characteristic absorption frequency $v_{\mathrm{e}}$ is constant, the absorption frequency in the SOA varies with the index of refraction such that, as the index increases, the oscillator energy decreases. This leads to index-based estimates of the Hamaker constant that are comparable to FS Hamaker constants.

$$
A_{121}=\frac{312\left(n_{1}^{2}-n_{2}^{2}\right)^{2} z J}{\left[\left(n_{1}^{2}-1\right)^{1 / 2}+\left(n_{2}^{2}-n_{1}^{2}\right)^{1 / 2}\right]\left(n_{1}^{2}+n_{2}^{2}\right)^{3 / 2}}
$$

$$
\varepsilon(i \xi)=1+\sum_{i=1}^{N} \frac{C_{i}}{1+\left(\frac{\xi}{\omega_{i}}\right)^{2}}
$$

where $C_{i}=(2 / \pi)\left(f_{i} / \omega_{i}\right)$ and $f_{i}$ the strength of the oscillator at frequency $\omega_{i}$. After various simplifying assumptions and approximations, they arrive at the following expression for the Hamaker constant:

$$
A_{121}^{\mathrm{SSM}}=\frac{3 k T}{2} \sum_{n=0}^{\infty}, \sum_{s=1}^{\infty} \frac{\left(\Delta_{12}\right)^{2 s}}{s^{3}}
$$

where $\Delta_{i j}$ are as in Eq. (26), but $\xi$ is replaced with $\xi_{n}=n(2 \pi k t / \hbar)$. $C_{i}$ can be calculated from the refractive index in the visible from a Cauchy plot, ${ }^{76}$ which gives, e.g., for a UV oscillator,

$$
n^{2}(\omega)-1=\left(n^{2}(\omega)-1\right) \frac{\omega^{2}}{\omega_{\mathrm{UV}}^{2}}+C_{\mathrm{UV}}
$$


Hence, with relatively little data, we can calculate approximations to Hamaker constants using a minimal amount of information on the material's electronic structure.

Bergström, ${ }^{30,82,83}$ among others, ${ }^{84}$ has used the SS method to estimate Hamaker constants for materials separated by interlayer materials of vacuum (air), water, $\mathrm{SiO}_{2}$, and hydrocarbon solvents. They calculated their $C_{i}$ oscillator parameters from optical data obtained using spectroscopic ellipsometry or from the literature and the Cauchy plot for the UV terms. The IR terms were calculated from the relation $C_{\mathrm{IR}}=\varepsilon(0)-C_{\mathrm{UV}}-1$. For ceramic materials, they typically used one IR and one UV term. For water, they used several oscillators and fitted them to various published spectral data of water.

(B) SS Representations of Water: The great importance of the LD forces in aqueous colloidal systems has made the SS representations of water much discussed in the literature by Bergström ${ }^{83}$ and others. ${ }^{85}$ Pashley $^{86}$ has compared the Hamaker constants for aqueous systems determined from the FS and SS methods and also from direct force measurement determinations. ${ }^{87}$ These aqueous Hamaker constant calculations are based on literature data for the spectral optical properties of water. ${ }^{88-92}$

(C) Index Approximations: For materials in which spectral optical properties are not available, the TWA and single oscillator (SOA) index approximations for the Hamaker constant provide useful estimates based on readily available indexes of refraction. Moreover, the variations of Hamaker constants with changes in the index of refraction of the grains or interlayers are very useful estimates of trends in LD forces.

The accuracy of TWA estimates is shown in a comparison of FS and TWA Hamaker constants. Figure 12 shows the Hamaker constants $A_{1 \mathrm{v} 1}^{\mathrm{NR}}$ for a vacuum gap between two identical materials, derived from the FS method and TWA. Agreement between FS and TWA Hamaker constants is within $15 \%$ for materials with an index of refraction in the range of 1.4-1.8. However, the TWA is increasingly poor for materials with higher indexes. Materials with higher indexes of refraction tend to have lower bandgap energies, which is neglected with the TWA's use of the constant $v_{\mathrm{e}}$ frequency. For higher-index materials, the SOA provides values for the Hamaker constant that are closer to the FS Hamaker constants.

(D) Simple Hamaker Constant Relationships: Various approximate relations have been proposed that relate values of various Hamaker constants, such as given in Eqs. (39)-(42).

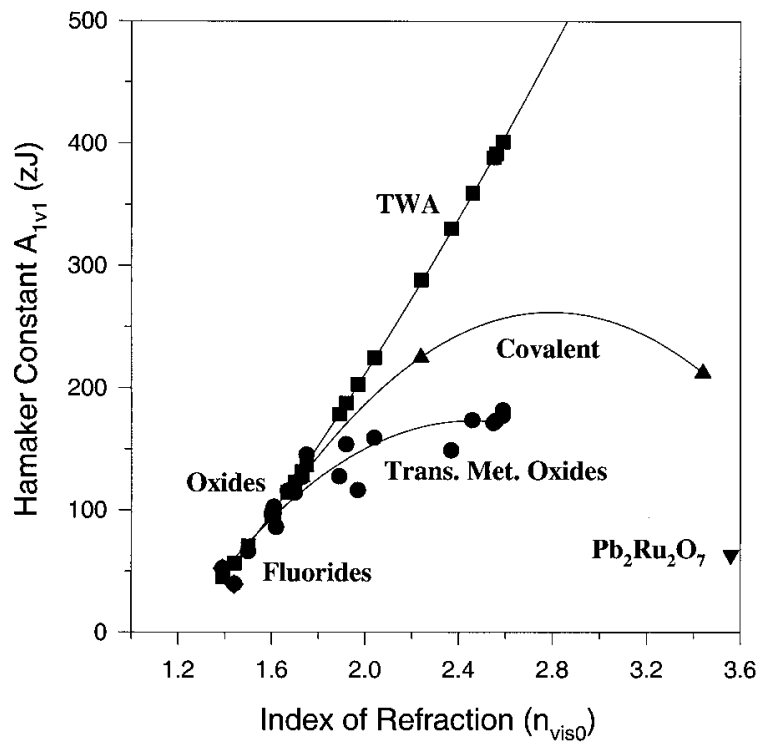

Fig. 12. $A_{1 \mathrm{v} 1}^{\mathrm{NR}}$ vs $n_{\text {vis } 0}$ for various materials calculated using FS calculations and the TWA. TWA results have been evaluated with a single value of $v_{\mathrm{e}}$ of $3 \times 10^{15} \mathrm{~Hz}$ for all materials. (Reprinted with permission from Elsevier Science. ${ }^{23}$ )

$$
\begin{aligned}
& A_{121}^{\mathrm{NR}}=A_{212}^{\mathrm{NR}} \\
& A_{123}^{\mathrm{NR}} \approx \pm\left(A_{121}^{\mathrm{NR}} A_{323}^{\mathrm{NR}}\right)^{1 / 2} \\
& A_{1 \mathrm{v} 3}^{\mathrm{NR}} \approx\left(A_{1 \mathrm{v} 1}^{\mathrm{NR}} A_{3 \mathrm{v} 3}^{\mathrm{NR}}\right)^{1 / 2} \\
& A_{121}^{\mathrm{NR}} \approx A_{1 \mathrm{v} 1}^{\mathrm{NR}}+A_{2 \mathrm{v} 2}^{\mathrm{NR}}-2 A_{1 \mathrm{v} 2}^{\mathrm{NR}}
\end{aligned}
$$

These types of relations, including other similar ones given by Israelachvili, ${ }^{29}$ can be of use with FS or estimated Hamaker constants if they are sufficiently accurate.

\section{(5) Retardation of Dispersion Forces and the Retarded Hamaker Constant $A^{\mathrm{R}}$}

The nonretarded Hamaker constant applies when the interparticle separation is very small compared with the wavelength of the interband absorptions of the interatomic bonds. When this nonretarded condition is met, the interactions among the induced dipoles can be considered instantaneous, and the photon transit time between the dipoles can be neglected.

When the interparticle separation is large, e.g., in the range of 5-10 nm, then the transit time of the virtual photons becomes important. The highest-energy interatomic bonds go out of phase with each other; therefore, their contribution to the LD interaction decreases. As the separation increases, the contributions of the high-energy bonds to the LD force are overestimated in the nonretarded Hamaker constant, and the retarded interaction $\left(A^{\mathrm{R}}\right)$ must be calculated.

To understand the effect of retardation, consider a material with interatomic bonds at interband transition energies of 7, 14, and 21 $\mathrm{eV}$. The energy of the interband transitions $\left(E_{\mathrm{ibt}}\right)$ and their wavelength $\left(\lambda_{\text {ibt }}\right)$ are given by Eq. (43) and in Table V. The critical interlayer thickness at which a particular interatomic bond dephases is estimated by Eq. (44). For this example, at a film thickness of $9 \mathrm{~nm}$, the $21 \mathrm{eV}$ transition energy bond no longer contributes to the dispersion force. When the film thickness reaches $14 \mathrm{~nm}$, then the $14 \mathrm{eV}$ transition energy bond no longer contributes. The details of the electronic structure and the interatomic bond energies dictate the details of the impact of retardation on the LD force.

$$
\begin{gathered}
\lambda_{\mathrm{ibt}}=\frac{h c}{E_{\mathrm{ibt}}} \\
L_{\text {crit }}^{\text {retard }} \approx \frac{\lambda_{\mathrm{ibt}}}{2 \pi}
\end{gathered}
$$

\section{Applications}

The LD forces are fundamental and ubiquitous, spanning all areas of science and technology. In this section we discuss various areas where dispersion forces play a role. Because LD forces are usually one of many forces and effects involved, the magnitude and effects of the dispersion forces compared with other forces and phenomena are examined for specific classes of materials. We look at dispersion forces in colloidal systems, interface energies, and wetting. Next, intersurface forces with a role in adhesion and sintering, which result from dispersion interactions, are discussed. We present the mechanical and electrical properties of a broad range of ceramic systems that exhibit intergranular glassy films mindful of the importance these films have acquired in the past 15 years for our understanding of ceramics processing and properties.

\begin{tabular}{lllll}
$\begin{array}{c}\text { Table V. Comparison of Interband Transition Energy and } \\
\text { Wavelengths, and Critical Thickness Estimates for } \\
\text { Retardation Effects in London Dispersion Forces }\end{array}$ & \\
\hline & Transition energy (eV) & 21 & 14 & 7 \\
\hline$E_{\mathrm{ibt}}$ & Transition wavelength (nm) & 59 & 89 & 177 \\
$\lambda_{\text {ibt }}$ & Critical thickness (mm) & 9 & 14 & 28 \\
$L_{\text {crit }}^{\text {retard }}$ & Crition & \\
\hline
\end{tabular}




\section{Panel C: Calculation of Full Spectral Retarded Hamaker Constants $A^{\mathbf{R}}$}

We calculate the retarded Hamaker spectrum, i.e., the Hamaker constant, as a function of thickness, following Elbaum and Schick. ${ }^{50}$ The free energy, per unit area, of an interlayer film of material 2 and thickness $L$ between two other materials 1 and 2 is given by

$$
\begin{aligned}
E(L)=\left[\frac{k T}{8 \pi L^{2}}\right] \sum_{n=0}^{\prime} \int_{\tau_{n}}^{\infty} \mathrm{d} x x\left\{\ln \left[1-\frac{\left(x-x_{1}\right)\left(x-x_{3}\right)}{\left(x+x_{1}\right)\left(x+x_{3}\right)} e^{-x}\right]\right. \\
+\ln \left[1-\frac{\left(\varepsilon_{3} x-\varepsilon_{2} x_{3}\right)\left(\varepsilon_{1} x-\varepsilon_{2} x_{1}\right)}{\left(\varepsilon_{3} x+\varepsilon_{1} x_{3}\right)\left(\varepsilon_{1} x+\varepsilon_{2} x_{1}\right)} e^{-x}\right]
\end{aligned}
$$

$$
x_{M}=\left[x^{2}-r_{\mathrm{n}}^{2}\left(1-\frac{\varepsilon_{\mathrm{M}}}{\varepsilon_{2}}\right)\right]^{1 / 2} \quad(M=1,3)
$$

$\varepsilon_{1}, \varepsilon_{2}, \varepsilon_{3}$ are the LD spectra of the materials, evaluated at imaginary frequencies. The sum $\Sigma_{n=0}^{\prime}$ is evaluated at the sequence of imaginary frequencies $\xi_{n}=(2 \pi k T / \hbar) n$. Combining this definition of frequency with the constant factor $k T / 8 \pi L^{2}$, we arrive at Eq. (47) for free energy of the LD interaction:

$$
\begin{aligned}
E(L)= & {\left[\frac{\hbar}{16 \pi^{2} L^{2}}\right] \sum_{n=0}^{\prime} \Delta \xi } \\
& \times \int_{r_{n}}^{\infty} \mathrm{d} x x\left\{\ln \left[1-\frac{\left(x-x_{1}\right)\left(x-x_{3}\right)}{\left(x+x_{1}\right)\left(x+x_{3}\right)} e^{-x}\right]\right. \\
& \left.+\ln \left[1-\frac{\left(\varepsilon_{3} x-\varepsilon_{2} x_{3}\right)\left(\varepsilon_{1} x-\varepsilon_{2} x_{1}\right)}{\left(\varepsilon_{3} x_{3}+\varepsilon_{2} x_{3}\right)\left(\varepsilon_{1} x+\varepsilon_{2} x_{1}\right)} e^{-x}\right]\right\}
\end{aligned}
$$

The sum $\Sigma_{n=0}^{\prime}$ over imaginary frequencies $\xi_{n}$ is evaluated at frequencies (or energies) separated by $\Delta \xi=2 \pi k T / \hbar$. When $T=300 \mathrm{~K}, \Delta \xi \approx 3.9 \times 10^{3} \mathrm{~Hz}(0.162 \mathrm{eV})$. We evaluate the sum over a finite region from 0 to $100 \mathrm{eV}$ instead of the infinite sum shown.

Each term of the sum, which is an integral over distance $\int_{r_{n}}^{\infty} \mathrm{d} x$ holding the frequency constant, is also approximated by a sum in our calculation. The sum extends from the lower limit of the integral $x=r_{n}=2 L \xi_{n}\left(\varepsilon_{2}\right)^{1 / 2} / c$ to an upper limit of $x=10$, where the exponential term $e^{-x}$ produces a negligible integrand. The sampling interval of the sum is $\Delta x=2000 /\left(10-r_{n}\right)$.

The algorithm for calculation of the retarded Hamaker constant is as follows:

- Choose a value of $L(L=0,1,2, \ldots)$ in units of 0.1 $\mathrm{nm}$, where $L$ is the thickness of the sandwich.

- Choose a frequency $\xi$ and obtain the value of the LD spectra of the three materials $\varepsilon_{1}(\xi), \varepsilon_{2}(\xi), \varepsilon_{3}(\xi)$.

- Prepare to do an inner integral (integral on $x$ ) for this frequency. Calculate the lower limit of integral, which is $2 \mathrm{~L} \xi\left(\varepsilon_{2}(\xi)\right)^{1 / 2} / c$. Evaluate the integral. If $L=0$, then multiply the results by one-half.

- Choose a new frequency and repeat the second step until all frequencies in the LD spectrum are satisfied.

This completes the calculation of the free-energy $E(L)$ for a single value of the interlayer thickness $L$. The calculation is repeated for subsequent interlayer thicknesses $L$. The Hamaker spectrum or Hamaker constant as a function of interlayer thickness is then calculated from the free energy as a function of thickness using Eq. (2). Finally, the force is calculated from the free-energy curve, $F(L)=-\Delta E(L) / \Delta L$, using numerical differences.
We then finish with a discussion of the effects of retardation on wetting, which can lead to unique wetting conditions.

\section{(1) Classes of Materials}

When assessing the importance of the dispersion forces in a particular problem, it is essential to consider the class of materials involved. In hydrocarbon materials and polymers, all the bonding is covalent and fully satisfied in the molecule or the polymer chain. These materials have very low levels of unsatisfied bonding, and their interfacial and surficial properties can be dominated by LD interactions. A result of the fully satisfied bonding in these materials is that surface energies tend to be low, and result predominantly from the LD interaction.

In ceramics and semiconductors, free surfaces typically have many unsatisfied atomic bonds. This disruption of the bulk electronic structure corresponds to thermodynamic energies that are much larger than the contribution resulting from the dispersion interactions. The surface energies of ceramics are much larger than polymers, and dispersion contributions, as shown below, are typically only $10 \%$ of the surface energy. In ceramics wet at high temperature by glasses, the unsatisfied chemical bonds of the ceramic grains can become satisfied, and then the dispersion interaction is critical. Silicates and other glass formers have a polymeric structure, and a high level of their atomic bonding is satisfied in the structural tetrahedra and the polymer chains. Silicate surfaces have lower levels of unsatisfied atomic bonds.

In hydrocarbons and hydrocarbon polymers, most chemical bonding is satisfied and dispersion is important. In aqueous systems, there are the effects of hydrogen bonding plus dispersion. In ceramics, ionic bonding is important, and in metallic systems there is metallic bonding.

\section{(2) Surface Energies, Wetting, and Contact Angles}

Because of the essential nature of surface energies and wetting, there have been many excellent discussions of their role in various materials systems and applications. Johnson ${ }^{95}$ focused on wetting of polymers by hydrocarbon solvents, a situation in which LD dominates. Israelachvili ${ }^{96}$ discussed the dispersion force contribution to works of adhesion and contact angles. There even has been recent discussion of the role of $\mathrm{vdW}$ forces in the adhesion of gecko foot hairs to surfaces. ${ }^{97}$ Zisman $^{98}$ and others ${ }^{99}$ compare wetting of both low- and high-energy solids, and they focus on the measurement of the contact angle, because this is a measurement of surface and interface energy. Clarke has discussed the role of dispersion and other forces, ${ }^{100}$ such as electrical double-layer forces, ${ }^{101}$ on the wetting of surfaces and interfaces (grain boundaries) in ceramic systems. ${ }^{102}$

The surface and interface energies of materials are related to unsatisfied bonding and to the dispersion effects from the optical contrast. It is these thermodynamic energies that help determine the wetting of solids by liquids. The topic of surface and interface energies, and also the contact angle at the triple point between the solid, liquid, and gas phases, shown in Fig. 13, was first studied by Young $^{2}$ and has been an active topic in science ever since. The Young equation (Eq. (48)) relates the contact angle to the surface and interface energies of the solid and liquid, through the use of a force balance at the interface. The closely related Young-Dupre equation (Eq. (49)) then defines the work of adhesion as the difference between the free surface energies of the solid and the liquid and the solid-liquid interface energy.

$$
\begin{aligned}
& \gamma_{\text {liquid }} \cos \theta=\gamma_{\text {solid }}-\gamma_{\text {solid-liquid }} \\
& W_{\text {ad }}=\gamma_{\text {solid }}+\gamma_{\text {liquid }}-\gamma_{\text {solid-liquid }}
\end{aligned}
$$

The thermodynamics of wetting has spanned from the work of Gibbs $^{6,7}$ and van der Waals ${ }^{5}$ to the critical wetting theory of $\mathrm{Cahn}^{103,104}$ and the development of the effective interface potential approach. ${ }^{105}$ The study has progressed to the detailed variations of three-dimensional droplets and their contact lines. ${ }^{106}$ In the more-complex issue of reactive wetting, e.g., of ceramics by metals at elevated temperature, the chemical reactions tend to 


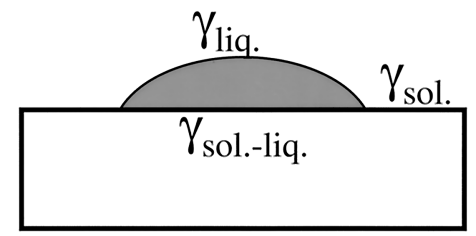

Fig. 13. Schematic drawing of a liquid droplet on a solid substrate $\left(\gamma_{\text {sol. }}\right.$ is the solid surface energy, $\gamma_{\text {liq. }}$ the liquid surface energy, and $\gamma_{\text {sol.-liq. }}$ the solid-liquid interface energy).

dominate the wetting process, and the dispersion forces can be considered negligible. A step beyond the equilibrium wetting condition is the process by which a wetting film dewets a surface, i.e., under what conditions a wetting film ruptures and dewets the substrate. ${ }^{107,108}$

Polymer-ceramic interactions are important in colloidal processing of ceramics and in engineering polymers, many of which are ceramic-particulate filled. In the case of milling and extrusion of ceramic-filled engineering polymers, we consider the dispersion contributions to the interface energies of polymers wetting $\mathrm{SiO}_{2}$ and $\mathrm{Al}_{2} \mathrm{O}_{3}$. Surface energies in most polymers result from the LD interaction. We can use experimental optical properties to determine their total surface energies. In Fig. 14 we show the interband transition strengths for five polymers. Polyester, polycarbonate, and polystyrene contain aromatic or conjugated phenyl groups that peak in the region from 4 to $7 \mathrm{eV}$, whereas poly(methyl methacrylate) (PMMA) and poly(ethylene-co-methacrylic acid) are only linear chain polymers and lack these lower-energy transitions.

From the interband transitions, we can calculate the LD spectra, and from these the Hamaker constants for $A_{1 \mathrm{v} 1}^{\mathrm{NR}}$ and $A_{121}^{\mathrm{NR}}$, where we choose material 2 as water, $\mathrm{SiO}_{2}$, and $\mathrm{Al}_{2} \mathrm{O}_{3}$. The Hamaker constant $A^{\mathrm{NR}}$ [PMMA $\left|\mathrm{Al}_{2} \mathrm{O}_{3}\right| \mathrm{PMMA}$ is $24.1 \mathrm{zJ}$, and, using Eq. (39), this also corresponds to the case of two grains of $\mathrm{Al}_{2} \mathrm{O}_{3}$ separated by a PMMA interlayer. Because all the Hamaker constants reported in Table VI are symmetrical, i.e., of the $A_{121}^{\mathrm{NR}}$ type, they are all attractive, and the values are all positive. The effects of optical contrast are evident for the case of water and $\mathrm{SiO}_{2}$ interlayers, where the index of the interlay and the polymeric grains are very similar, the optical contrast in the system is very low, and the LD interaction and Hamaker constants are small.

On calculating the necessary $A_{1 \mathrm{v} 3}^{\mathrm{NR}}$ Hamaker constants, we can determine the dispersion contribution to the interface energies for the polymer-ceramic systems, as are shown in Table VII. The

\section{Panel D: Interface Energies and Contact Angles for Droplet on Substrate}

Because the Hamaker constant represents the LD interaction energy of two materials, it gives us the dispersion contribution to surface and interface energies. If dispersion forces are the main contribution to the interface energies, we can also calculate the contact angle. Let us consider a $A^{\mathrm{NR}}$ Hamaker constant for a material, which represents the LD interaction for two grains of material 1 separated by vacuum. Let us also assume for now that this is a material, such as a polymer, in which the surface energy is determined by the dispersion interactions. If we evaluate the Hamaker constant at a value $d_{\mathrm{o}}$, which corresponds to an equilibrium separation, such as an interatomic bond length, then we have calculated twice the surface energy of material 1. Typically, the value of $d_{\mathrm{o}}$ is taken to be $0.165 \mathrm{~nm}^{29}$ Similarly, if we evaluate the $A_{1 \mathrm{v} 3}^{\mathrm{NR}}$ Hamaker constant between two different materials 1 and 3 for an appropriate $d_{\mathrm{o}}$ separation, again separated by vacuum, then we have calculated the interface energy for an interface of material 1 and 3. To calculate the surface and interface energies, as shown in Fig. 13, requires the calculation of $A^{\mathrm{NR}}$ [liquid|vacuum|liquid] and $A^{\mathrm{NR}}$ [solid|vacuum|liquid], then using Eqs. (50) and (51), resulting in $\gamma_{\text {liquid. }}$ and $\gamma_{\text {solid-liquid. }}$

$$
\begin{aligned}
& \gamma_{\text {material } 1}=-\frac{1}{2} E_{\text {material1 }}=\frac{A_{1 \mathrm{v} 1}}{24 \pi d_{\mathrm{o}}^{2}} \\
& \gamma_{\text {material1 material3 }}=-\frac{1}{2} E_{\text {material1 material3 }}=\frac{A_{1 \mathrm{v} 3}}{24 \pi d_{\mathrm{o}}^{2}}
\end{aligned}
$$

These correspond to the LD contribution to the interface energies. In materials classes where Eqs. (50) and (51) correspond to the total interface energies, then we can use the Young equation (Eq. (48)) to determine the contact angle $\theta$, which in terms of Hamaker constants and assuming a constant value of $\mathrm{d}_{\mathrm{o}}$, is given in Eq. (52).

$$
\theta=\arccos \left(\frac{A_{121}}{A_{123}}-1\right)
$$

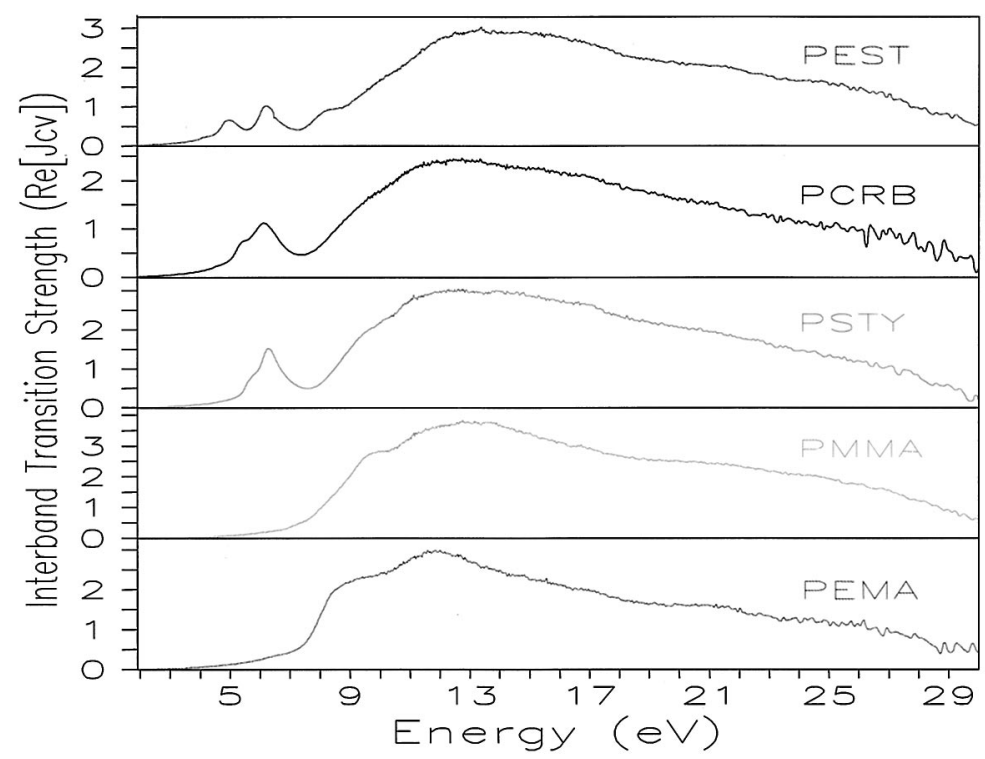

Fig. 14. Interband transition strengths, $J_{\mathrm{cv}}$, of polyester (PEST), polycarbonate (PCRB), polystyrene (PSTY), poly(methyl methacrylate) (PMMA), and poly(ethylene-co-methacrylic acid) (PEMA) determined from VUV reflectivity. 
Table VI. Refractive Indexes and Hamaker Constants $\left(A_{121}^{\mathbf{N R}}\right)$ for Various Polymers ${ }^{\dagger}$

\begin{tabular}{lccccc}
\hline $\begin{array}{c}\text { Polymer } \\
\text { refractive index }\end{array}$ & $\begin{array}{c}\text { Polymer } \\
(\text { material 1) }\end{array}$ & $\begin{array}{c}A^{\mathrm{NR}}(\mathrm{zJ})(\text { vacuum }) \\
(n=1.0)\end{array}$ & $\begin{array}{c}A^{\mathrm{NR}}(\mathrm{zJ})(\text { water }) \\
(n=1.33)\end{array}$ & $\begin{array}{c}A^{\mathrm{NR}}(\mathrm{zJ}) \text { (silica) } \\
(n=1.54)\end{array}$ & $\begin{array}{c}A^{\mathrm{NR}}(\mathrm{zJ}) \text { alumina } \\
(n=1.76)\end{array}$ \\
\hline $1.4893^{230}$ & PMMA & 58.4 & 1.47 & 0.634 & 24.1 \\
$1.51^{231}$ & ET-MAA & 47.7 & 2.16 & 2.71 & 33.0 \\
$1.64-1.67$ & PEST & 60.9 & 4.05 & 1.94 & 25.4 \\
1.70 & PIMI & 62.6 & 5.23 & 2.61 & 32.6 \\
$1.586^{232}$ & PCARB & 50.8 & 3.5 & 3.08 & 28.3 \\
$1.590-1.592^{230}$ & PSTY & 55.6 & 3.16 & 2.04 & 2.4 \\
\hline
\end{tabular}

${ }^{\dagger}$ Polymer is defined as material 1 , and vacuum, water, $\mathrm{SiO}_{2}$, and $\mathrm{Al}_{2} \mathrm{O}_{3}$ are defined as material 2. By definition, $A_{121}^{\mathrm{NR}}=A_{212}^{\mathrm{NR}}$.

Table VII. Dispersion Contributions to Surface and Interface Energies ${ }^{\dagger}$

\begin{tabular}{lccc}
\hline Material & $\begin{array}{c}\text { Surface energy } \\
\left(\mathrm{mJ} / \mathrm{m}^{2}\right)\end{array}$ & $\begin{array}{c}\text { Interface energy on } \\
\mathrm{SiO}_{2}\left(\mathrm{~mJ} / \mathrm{m}^{2}\right)\end{array}$ & $\begin{array}{c}\text { Interface energy on } \\
\mathrm{Al}_{2} \mathrm{O}_{3}\left(\mathrm{~mJ} / \mathrm{m}^{2}\right)\end{array}$ \\
\hline PMMA & $29(41.1)$ & 62 & 89 \\
ET-MMA & 24 & 55 & 80 \\
$\mathrm{PEST}$ & $30(44.6)$ & 62 & 90 \\
$\mathrm{PIMI}$ & $30(41)$ & 63 & 90 \\
$\mathrm{PCARB}$ & $25(33)$ & 57 & 82 \\
$\mathrm{PSTYR}$ & $27(40.7)$ & 60 & 86 \\
$\mathrm{Al}_{2} \mathrm{O}_{3}$ & 71 & & \\
$\mathrm{SiO}_{2}$ & 33 & & \\
\hline &
\end{tabular}

tabulated surface energies of the polymers are very low, in the range of $25-30 \mathrm{~mJ} / \mathrm{m}^{2}$, and correspond well to independently determined values of the surface energies. ${ }^{109,110}$ For $\mathrm{Al}_{2} \mathrm{O}_{3}$, the dispersion force contributions to the surface energies correspond to only $5 \%$ of the measured surface energy, because the unsatisfied bonding on the surface of $\mathrm{Al}_{2} \mathrm{O}_{3}$ contributes a very large thermodynamic energy to the surface energy, dwarfing the contributions resulting from dispersion.

\section{(3) Balance of Forces}

Just as consideration of the material classes involved are important, other compensating forces usually effect systems as well as the dispersion interactions. For example, an attractive dispersion force typically is counterbalanced by a repulsive force, such as a hard-core repulsion, when two atoms approach. The outcome is shown in Fig. 15, where the force balance of these two forces leads to a total force, with an equilibrium point resulting where the force is zero. This concept of the force balance, shown in Eq. (53), is common to many applications of dispersion forces, and the forces involved can result from electrical double layers (EDL), adsorption (Ads.), hydrogen bonding (HB), and steric forces (St.), among others.

$$
F=0=F_{\mathrm{LD}}+F_{\mathrm{EDL}}+F_{\mathrm{ads}}+F_{\mathrm{HB}}+F_{\mathrm{St}}
$$

\section{(4) Colloidal Systems}

Colloidal systems of polymer- or ceramic-particulate dispersions serve as critical role in ceramic processing as has been discussed recently in detail by Lewis. ${ }^{111}$ The LD forces are a fundamental attractive force involved in colloidal dispersions, ${ }^{33,112}$ and it is the detailed balance of this and other forces that dictates the behavior of the particulate dispersion. ${ }^{22}$ Derjaguin and Landau ${ }^{12,113}$ and Verwey and Overbeek ${ }^{33}$ (DLVO) developed DLVO theory from the perspective of the force balance of Eq. (53), which is the basis of understanding and controlling the interparticle forces and thereby colloidal dispersions stability and rheology.

\section{(5) Interparticle Forces}

The direct measurement of the interparticle force balance has been a long-standing focus of research, because LD forces and the

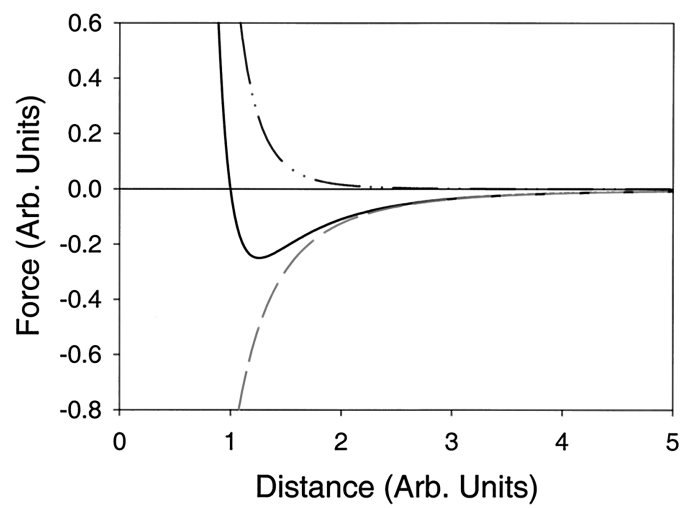

Fig. 15. Force-distance curve for $(---)$ LD force, $(-\cdot--)$ repulsive force, and (-) total force.

force balance can allow stable equilibrium behavior in colloidal systems. DLVO theory ${ }^{33}$ has provided a method to estimate the contributions from the LD force and EDL force, among others, to interpret measured force-distance relations for two planar bodies, a sphere or cylinder approaching a plane, two crossed cylinders, or two spheres. With this theoretical underpinning, work commenced in the 1950 s on developing quantitative tools to directly measure the interparticle forces. The surface force apparatus (SFA), which began development in the 1950 s, has been joined in the 1980 s by the atomic force microscope (AFM).

(A) Surface Force Apparatus: In the 1950s, Overbeek ${ }^{49,112}$ and Derjaguin ${ }^{22,114}$ began measuring interparticle forces and force-distance relations using plane-parallel plates; they later had more success using curved surfaces, which simplified alignment of the two materials. They introduced optical interferometric techniques to accurately measure the separation of the two materials, as long as they were transparent. These initial results tended to demonstrate forces much larger than expected for the LD interaction, but with sufficient care, dispersion forces at separations of $100 \mathrm{~nm}$ were measured. With the work of Tabor and Winterton ${ }^{93}$ and Israelachvili, ${ }^{115}$ the SFA, based on a crossed cylinder geometry, became a well-established instrument for measuring the intersurface force-distance relations for mica under vacuum or a wide variety of liquids. Mica is the most common material used in the SFA because of the ease of preparing an atomically smooth surface and adhering it to cylindrical substrates. With additional work at preparing curved surfaces of additional materials, such as $\mathrm{Al}_{2} \mathrm{O}_{3},{ }^{116}$ the range of materials studied using the SFA expanded. A large amount of the SFA research was focused on measurement of the various force terms in DLVO theory for complex liquid systems. ${ }^{117}$ Moreover, the effects of the molecular ordering of organic solvents ${ }^{118}$ and water ${ }^{119}$ in the interlayer have been studied at very close approach.

(B) Atomic Force Microscope: The development of the $\mathrm{AFM}^{120}$ dramatically expanded the types of materials for which direct intersurface force measurements ${ }^{121}$ could be determined. The AFM has permitted the study of DLVO forces, ${ }^{122}$ such as EDL and hydration. ${ }^{123}$ The opportunity of AFM to measure the LD forces with vacuum or liquid interlayers is complicated, because the complex shape of the tip affects the analysis of the 


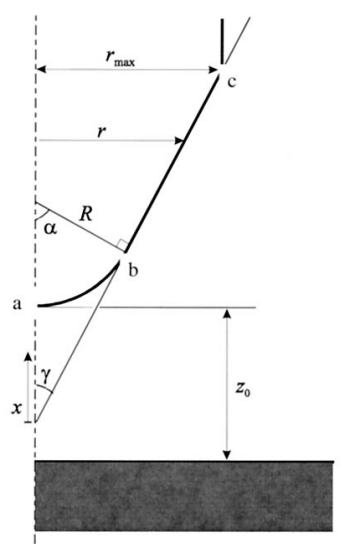

Fig. 16. Parametric tip model. $R$ is the tip radius, $r_{\max }$ the radius of the cylindrical part of the probe, $\gamma$ the cone angle, $\alpha=\pi / 2-\gamma$ the angle included in the spherical cap, $z_{0}$ the probe-sample separation, $r$ the radius at any point on the surface, $x$ the distance from the cone apex, and $a, b$, and $c$ define the spherical cap section and the conical section of the probe.

force-distance relation to extract the Hamaker constant. This is where the simple crossed cylinders geometry of the SFA is an advantage. Initial work by Hartmann ${ }^{124}$ and others and recent application of the intersurface stress tensor approach (discussed in more detail in Section III(6)) now permits the extraction of Hamaker constants from measured force-distance relations, ${ }^{125}$ assuming the LD forces dominate. The parametric tip forcedistance relation (Eq. (54)) permits the nonlinear fitting of the measured LD force to the Hamaker constant $A^{\mathrm{NR}}$ and the geometrical parameters of a conical AFM tip, with a spherical cap (shown in Fig. 16).

$$
\begin{aligned}
F_{z}\left(z_{0}\right)= & \frac{A R^{2}(1-\sin \gamma)\left(R \sin \gamma-z_{0} \sin \gamma-R-z_{0}\right)}{6 z_{0}^{2}\left(R+z_{0}-R \sin \gamma\right)^{2}} \\
& +\frac{-A \tan \gamma\left(z_{0} \sin \gamma+R \sin \gamma+R \cos 2 \gamma\right)}{6 \cos \gamma\left(z_{0}+R-R \sin \gamma\right)^{2}}
\end{aligned}
$$

AFM tips are most commonly fabricated from $\mathrm{Si}_{3} \mathrm{~N}_{4}$, and many studies of intersurface forces in $\mathrm{Si}_{3} \mathrm{~N}_{4}$ have been reported. ${ }^{126} \mathrm{By}$ the attachment of a colloidal particle of a material to the AFM tip, the ability to measure intersurface forces between many colloidal particles and an arbitrary substrate material has become one of the most fruitful applications of the AFM in colloid science. Intersurface force measurements in aqueous or nonaqueous systems have now been done for $\mathrm{TiO}_{2},{ }^{127}$ gold, ${ }^{128} \mathrm{Al}_{2} \mathrm{O}_{3},{ }^{129} \mathrm{ZrO}_{2},{ }^{130,131}$ ice, ${ }^{132}$ and other materials.

\section{(6) Adhesion and Sintering}

The LD forces can be approached in different ways, e.g., at the discrete point-to-point approach of Hamaker for atoms and molecules or at the continuum level; the continuum approach of Lifshitz is the most useful for the formulation and analysis of "macroscopic" problems. There are two important macroscopic manifestations of molecular interactions: interactions within a body that result in surface tension and interactions between bodies that affect phenomena such as adhesion and the behavior of colloids. Often, only one of the macroscopic effects of molecular interactions is considered. For example, in the cohesive zone theory of fracture mechanics, ${ }^{133}$ forces behind the crack tip are assumed to be based on interactions across the gap. As such, the forces are oriented normal to the free surface. On the other hand, forces at a sintering neck, which is essentially an exterior crack, usually have been modeled based on surface tension and local curvature. ${ }^{134}$ The difference between the two is illustrated in Fig. 17. Both sets of forces are energetically consistent with the intermolecular forces on which they are based in that both yield the same work per unit extension of surface area. However, because they are so different in detail, they lead to very different kinetics. In many problems, one of the macroscopic effects of molecular interaction is sufficient to model surface-force-driven processes. However, in the problem of viscoelastic sintering, viscous sintering of fine particles, and viscoelastic crack growth, we must consistently describe the in-plane surface tension and the normal dispersion force or "interaction across a gap."135

The continuum treatment of surface tension yields the notion of surface stress. For liquids, the associated pressure $p$ on a surface is proportional to curvature $\mathrm{\kappa}$ via Eq. (55), developed by Young and Laplace, ${ }^{136}$

$$
p=\gamma \kappa=\gamma\left(\frac{1}{R_{1}}+\frac{1}{R_{2}}\right)
$$

where $R_{1}$ and $R_{2}$ are the principal radii of curvature. Two different approaches have been followed in the continuum treatment of interactions across a gap. The more general is that of Lifshitz et $a l .{ }^{19}$ in which interactions are calculated in terms of virtual photons and electromagnetic fluctuations and interactions between half spaces. This approach relates interaction forces to continuum properties of the interacting materials, including those that fill the gap.

The Lifshitz approach is based on the recognition that the use of the point-to-point interaction energy is too simplistic to compute
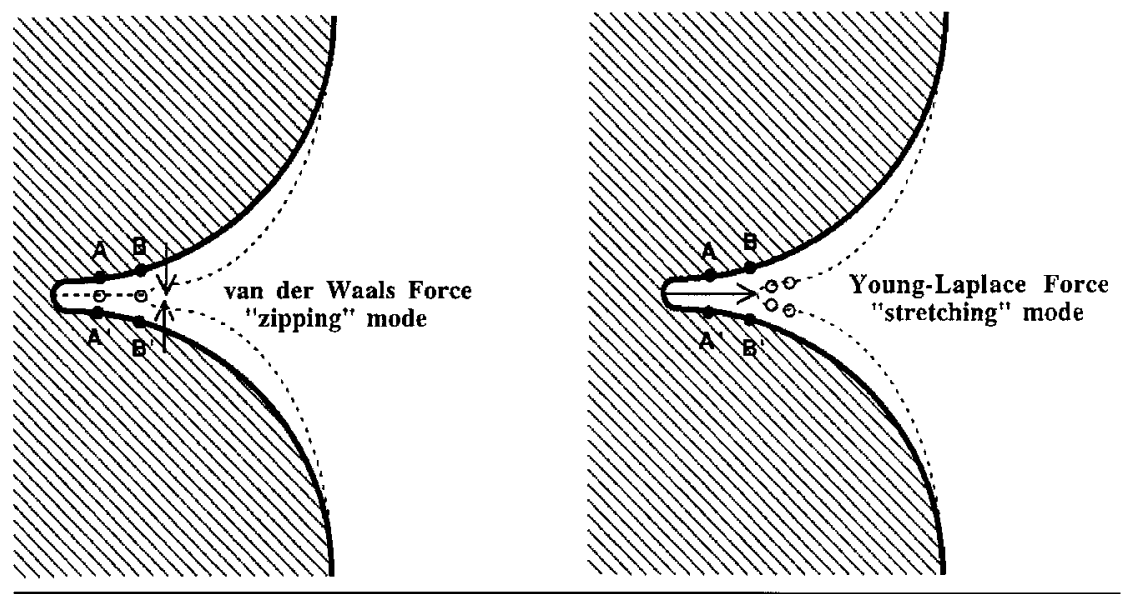

Fig. 17. Schematic illustration of contact growth modes in elastic adhesion, which is driven by direct attraction across the gap ahead of the contact neck, and viscous sintering that is driven by tractions that are modeled as being proportional to the surface curvature. For elastic adhesion, the contact grows in a zipping mode, whereas, for viscous sintering, the contact grows in a stretching mode. ${ }^{134}$ 
interactions between macroscopic solid bodies of atoms or molecules with a high density of packing. Not incorporating this fact, Hamaker's approach ${ }^{12}$ postulates that the total interaction between solid bodies can be computed by integrating pairwise interactions between infinitesimal individual regions, and these interactions are assumed to be central, i.e., aligned along the vector connecting them. The power of this assumption is that it is possible to calculate interactions between various-shaped bodies. For interactions between identical materials with no intervening medium (vacuum), the functional form of the interaction is correct, but the Hamaker constant cannot be related directly to the intermolecular interaction energy. ${ }^{137}$ There are several other cases where the Hamaker assumption of pairwise additivity breaks down completely, i.e., in computing interactions between two materials with a third intervening medium. The Lifshitz approach has been detailed in Section II(2); in the following paragraphs we develop the pairwise additive approach for interaction between bodies of general shape.

Let the pairwise interaction energy of two molecules as a function of distance $d$ be $w(d)$. The interaction energy $e(d)$ between two elemental volumes $\mathrm{d} V_{1}$ and $\mathrm{d} V_{2}$ then is

$$
e(d)=\rho_{1} \rho_{2} w(d) \mathrm{d} V_{1} \mathrm{~d} V_{2}
$$

where $\rho_{1}$ and $\rho_{2}$ are the number densities of molecules. These infinitesimal volumes interact, each with every other, and it is assumed that the total energy of the system is the sum of individual interaction energies. The interaction energy $E$ between two bodies defined by the volumes $V_{1}$ and $V_{2}$ is then given by the double volume integral

$$
E=\int_{V_{2}} \int_{V_{1}} \rho_{1} \rho_{2} w(d) \mathrm{d} V_{1} \mathrm{~d} V_{2}
$$

Similarly, the total force of interaction between two bodies can be computed.

$$
\hat{F}=\int_{V_{2}} \int_{V_{1}} \rho_{1} \rho_{2} \hat{f}(d) \mathrm{d} V_{1} \mathrm{~d} V_{2}
$$

where $\hat{f}(d)$ is the interaction force given by the spatial gradient $\nabla(\cdot)$ of the interaction energy as

$$
\hat{f}(d)=-\nabla w(d)
$$

Vectors are denoted by a caret (e.g., $\hat{f}$ ) and second rank tensors by a double caret (e.g., $\hat{h}$ ). Interactions handled in this manner have been computed for various geometries. ${ }^{29}$ If

$$
w(d)=-\frac{A^{\mathrm{NR}}}{\pi^{2} \rho_{1} \rho_{2} d^{6}}
$$

which is the vdW interaction energy for point-to-point interactions, then the interaction energy of two half spaces separated by a distance $d$ reduces to Eq. (2) where $m=2$ and $A^{\mathrm{NR}}$ is the Hamaker constant.

The application of the point-to-point summation approach remains difficult for arbitrarily shaped bodies. Derjaguin ${ }^{138}$ introduced an approximation that is accurate when surface radii of curvature are large compared with the gap between the bodies. It consists of treating opposing surfaces as composed of elements that interact as would half spaces. Formally, surface traction $\hat{T}$, a force per unit area, is then computed as

$$
\hat{T}=F(d)(\hat{q} \cdot \hat{n}) \hat{q}
$$

where $F(d)$ is the interaction force (per unit area) between two half spaces, $d$ the distance between the surfaces measured along $\hat{q}$, and $\hat{n}$ the surface normal.

Hamaker's approach for the computation of interactions between bodies has been generalized recently to account for arbitrary geometry. ${ }^{135,139}$ Consider two bodies 1 and 2 as shown in Fig. 18.
Body 1 causes distributed forces $\hat{b}$ (per unit volume) in body 2, given by,

$$
\hat{b}=\rho_{1} \rho_{2} \int_{V_{2}} \nabla_{2}(w(d)) \mathrm{d} V_{2}=\rho_{1} \rho_{2} \int_{S_{2}} w(d) \hat{n}_{2} \mathrm{~d} S_{2}
$$

where $\mathrm{d} V$ is a volume element and $\mathrm{d} S$ a surface element.

Following a special integration procedure, these can be replaced by effective surface tractions $\hat{T}$ on the surface of body 2 . Then, the influence of body 1 is represented by an intersurface stress tensor $\hat{h}$ such that

$$
\begin{aligned}
& \hat{T}=\hat{\hat{h}} \cdot \hat{n}_{1} \\
& \hat{\hat{h}}=\rho_{1} \rho_{2} \int_{S_{2}} \hat{n}_{2} \hat{G} \mathrm{~d} S_{2}
\end{aligned}
$$

where $\hat{G}$ is the vector field

$$
\hat{G}=\left(\hat{x}_{2}-\hat{x}_{1}\right) v(d)
$$

and $\hat{x}_{1}$ and $\hat{x}_{2}$ are position vectors to surfaces 1 and 2 . The scalar function of distance between the two surfaces, $v(d)$, is derived from the intermolecular interaction energy:

$$
v(d)=\frac{1}{d^{3}} \int_{d}^{\infty} w(t) t^{2} \mathrm{~d} t
$$

Therefore, the external influence of body 1 is represented by $\hat{\hat{h}}$. If another surface is introduced into the field of $\hat{h}$, it produces a traction on that surface in a manner analogous to the internal stress. The total force of interaction on body 1 because of body 2 is equal and opposite to that on body 2 because of body 1 . However, unlike in Derjaguin's approximation, the distribution of forces on the two bodies may be very different.

It has been pointed out ${ }^{140}$ that the stress $\hat{h}$ is formally equivalent to the Maxwell stress of electromagnetic theory, ${ }^{141}$ in that its divergence yields the distributed body force (or force per unit volume). Using this construction, an alternative method for solving this class of problems has been developed.

As mentioned earlier in this section, sintering is usually analyzed using only the surface tension representation (Fig. 17(a)) of surface forces. ${ }^{142,143}$ However, based on the Johnson-KendallRoberts theory of adhesion between elastic spheres, ${ }^{144}$ which neglects the surface tension aspect but includes the attraction across a gap (Fig. 17(b)), all sintering begins by the formation of a neck due to elastic deformations, the so-called zeroth stage of sintering. ${ }^{142}$ Both manifestations of surface forces act simultaneously at a sintering neck. Moreover, there may be a regime of particle size in which sintering is controlled by direct attractive forces across the gap ahead of the neck, rather than by surface tension. ${ }^{145}$ By combining both surface tension and direct attractive interactions, Jagota et al. ${ }^{134}$ have studied the problem of sintering of a Maxwell viscoelastic sphere to a rigid half space. Their analysis retrieves the known limits: initial elastic adhesion driven by direct attractive forces and final contact growth driven by surface tension. For submicrometer particles, a new mode of viscous sintering develops, which is driven by direct attractive

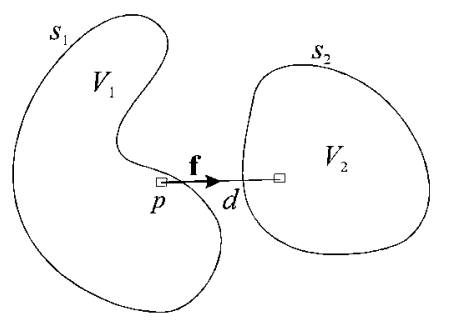

Fig. 18. Two arbitrarily shaped bodies. ${ }^{137}$ 
forces. In it, the contact "zips" shut in contrast with the conventional sintering calculations, where the contact is stretched open. Classical Herring scaling between sintering time and particle radius breaks down as a new length scale, the range of cohesive forces, enters into the relationship. Scaling of contact radius with time, ${ }^{146,147}$ instead of $t^{1 / 2}$ given by the Frenkel model, is between $t^{1 / 5}$ and $t^{1 / 7}$. These predictions remain to be verified experimentally for viscous sintering; however, the zipping mode of contact growth explains well the observed sintering kinetics in polymeric systems that have more-complex rheology. ${ }^{148}$

\section{(7) Equilibrium Intergranular and Surficial Films}

The properties of liquid-phase sintered or liquid-infiltrated materials depend on the characteristics of the intergranular phases and associated interfacial films (as shown in Fig. 19) as well as the morphologies of the bulk phases. ${ }^{149,150}$ Many studies have pointed out that the amount and composition of the sintering aids influence the microstructure evolution during sintering, ${ }^{151-154}$ thereby affecting the chemical and physical properties of the final microstructures. The actual interfacial features also strongly influence mechanical and physical properties, ${ }^{155}$ e.g., creep resistance ${ }^{156,157}$ or fracture toughness ${ }^{158-162}$ of $\mathrm{Si}_{3} \mathrm{~N}_{4}$ - or $\mathrm{SiC}$-based materials, or the electrical properties in thick-film resistors, ${ }^{163}$ varistors, ${ }^{164}$ or high- $T_{\mathrm{c}}$ superconductors. ${ }^{165}$ In all these cases, the presence of intergranular films and the specific impurities in them profoundly alter macroscopic properties, often beneficially. Thus, it is important that the actual composition and bonding of the interfacial films can differ from those of the associated bulk glass phase. ${ }^{163-171}$ The factors controlling the composition and structure of these films are a matter of current research, but the dispersion forces across them are one of the important factors controlling their stability.

Numerous investigations have focused on features affecting the sintering process of materials with low liquid contents. Densification is frequently expedited by the presence of a small liquid fraction. The driving force is usually described in terms of the effect of capillary pressure on the grain-boundary formation process. ${ }^{172-174}$ The capillary forces contemplated result from the menisci at liquid-vapor interfaces between contacting particles that drive sintering by pulling particles together. An important concept is that, despite the compressive force induced across particle contacts, enough of the liquid-forming material must remain adsorbed at the boundaries to provide the high boundary diffusion required to account for enhanced rates of densification via grain-boundary formation. ${ }^{171,175}$ In $\mathrm{Si}_{3} \mathrm{~N}_{4}$-based materials, boundaries with 1-2 nm thick, amorphous oxynitride films usually form between particles during liquid-phase sintering. ${ }^{176-178}$ Moreover, it has been shown that the boundary thickness is almost constant in a given material although it adjusts depending on the

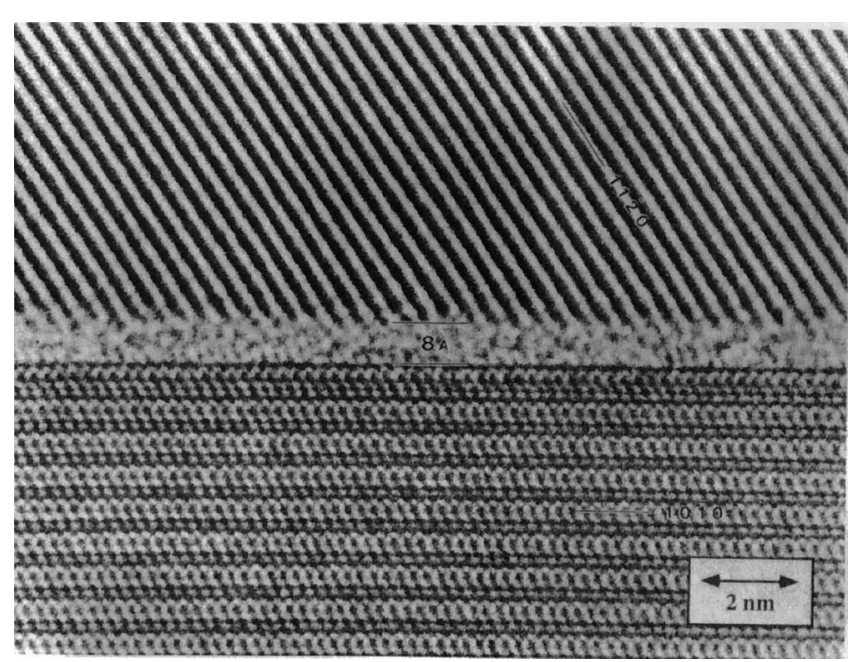

Fig. 19. HRTEM image of an equilibrium intergranular film in $\mathrm{Si}_{3} \mathrm{~N}_{4} \cdot{ }^{155,179}$ exact composition, ${ }^{155,179-181}$ implying that the thickness reflects some equilibrium condition. ${ }^{182,183}$ However, under conventional sintering conditions in some systems, e.g., $\mathrm{ZnO}-\mathrm{Bi}_{2} \mathrm{O}_{3}$, the capillary forces reversibly influence the grain-boundary composition; hence, it is not only a result of an equilibrium among local forces acting across a boundary or between two solid-liquid interfaces. ${ }^{170,184}$

Complementary approaches to probe the particle interactions and the roles of interfacial composition begin with considering the solid phase (either powder or dense polycrystal, initially) being immersed in a massive amount of liquid at high temperature, as demonstrated by Shaw and others. ${ }^{185-188}$ Such systems largely evolve in the absence of the capillary forces induced by liquidvapor interfaces, under conditions where dispersion forces, and also surface energies, can be dominant. Consider when a small amount of refractory particles is immersed at high temperature in a liquid matrix: A dilute suspension is realized. This can be treated as a colloidal system, where local forces among particles govern their mutual interactions. A wide range of behaviors can prevail in which the specific particle-particle interaction influences the degree of interconnectedness and the types of grain boundaries that develop between particles. ${ }^{188,189}$ These features then influence the microstructural evolution via the strength of the solid network, as well as through the interfacial kinetic parameters. Alternatively, if a dense polycrystalline material is immersed in excess liquid at high temperature, liquid penetration along the triple points and possibly through grain boundaries can eventually occur, as has been observed for $\mathrm{Al}_{2} \mathrm{O}_{3}$ in silicates. ${ }^{185,188}$

If a more global understanding of the underlying interparticle forces, interfacial reactions, and kinetics emerges, it should be feasible to design and fabricate a richer variety of materials with superior properties. For example, the boundary penetration process has profound implications concerning the corrosion or oxidation resistance of ceramics, and it can be beneficially used to form functionally graded materials. ${ }^{188}$ Graded materials made with $\mathrm{Al}_{2} \mathrm{O}_{3}$ or $\mathrm{Si}_{3} \mathrm{~N}_{4}$ partially penetrated by silicates have been shown to have enhanced resistance to wear and contact damage. ${ }^{190,191}$ Moreover, based on this principle, a transient liquid can be used to promote brazing or diffusion bonding between pieces of ceramic leading to seamless joints, ${ }^{192,193}$ where the liquid is imbibed into the bulk ceramic pieces.

(A) Various Oxide Systems: In many ceramic systems sintered with various impurities and dopants, a liquid phase exists at temperature and is often manifest by residual glassy phases or grain-boundary films in the as-cooled materials. Evidence of continuous silicate-rich or other amorphous phases and films have been seen in a wide variety of oxide, ${ }^{194,195}$ nitride, ${ }^{196,197}$ and carbide ${ }^{160}$ ceramics. These include materials, such as $\mathrm{MgAl}_{2} \mathrm{O}_{4}$, in which the fluoride-rich sintering aid leads to a continuous boundary phase. ${ }^{198}$ Recently, it has been appreciated that the amorphous grain-boundary films in some systems have an equilibrium thickness, which indicates that these are adsorption films that formed in equilibrium with some second phases at temperature. More recently, it has been found that these films have composition different from the high-temperature liquids with which they were in equilibrium. Thermodynamically, these films can be described in terms of Gibbsian adsorption. ${ }^{105}$ However, an atomistic description can be considered in terms of a force balance across the film, which must involve gradients across the film as well as differences in composition between the film and the second-phase regions. This fundamentally includes the dispersion forces across the film. Simpler descriptions, which have a strong parallel with lowtemperature colloidal theories, have included a variety of disjoining forces with the assumption that the primary attraction force is the dispersion force. The neglect of other short-range attractive forces may be an important deficiency, but, for thicker films, it is likely that the dispersion force is the largest attractive force.

Thus, based simply on trends in dispersion forces (Table VIII), it has been expected that multilayer boundary films should be stable and thick for grains of low-index oxides, such as $\mathrm{MgO}$ or $\mathrm{Al}_{2} \mathrm{O}_{3}$, together with silicate liquids. ${ }^{182}$ Indeed, for $\mathrm{Al}_{2} \mathrm{O}_{3}$, there are many observations of extensive glassy pockets and films in 
Table VIII. Nonretarded Hamaker Constants for Various Ceramic Systems ${ }^{\dagger}$

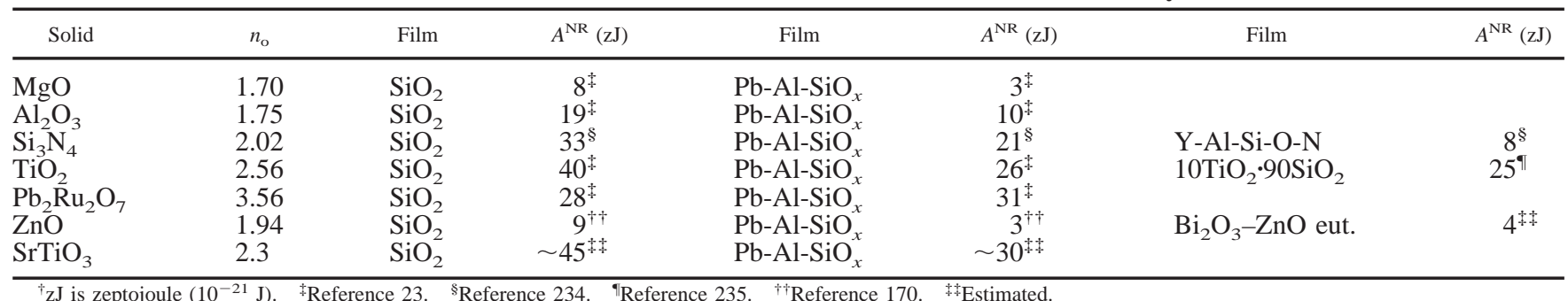

polycrystals. ${ }^{194,199-203}$ Moreover, for $\mathrm{Al}_{2} \mathrm{O}_{3}$ (both pure and containing various amounts of $\mathrm{CaO}, \mathrm{SiO}_{2}$, or other impurities) extensive penetration of dense polycrystals by silicate liquids occurs, leading eventually to widespread disruption, i.e., deflocculation of the grains, which may mean the dihedral angle is low or the liquid is virtually wetting. ${ }^{185,204} \mathrm{Al}_{2} \mathrm{O}_{3}$ powders initially mixed into glasses give similar results, in which particles remain widely separated, but do concentrate somewhat while settling because of gravity. ${ }^{205}$ However, for $\mathrm{Al}_{2} \mathrm{O}_{3}$ and $\mathrm{MgO}$, which can be extensively penetrated by silicate liquids, ${ }^{206}$ a discernable fraction of boundaries is not wetted or obviously penetrated. The condition of these boundaries, i.e., whether moist, dry, or special, has not been assessed by analytical or high-resolution TEM. However, TEM studies of $\mathrm{Al}_{2} \mathrm{O}_{3}$ polycrystals with small levels of glass indicate that the dry boundaries are typically special, often twins. ${ }^{199,200,203}$ Indeed, there has been comparatively little characterization of the actual films in most studies of these two oxides.

Similarly, it was found that iron-doped $\mathrm{SrTiO}_{3}$ polycrystals having excess $\mathrm{TiO}_{2}$ exhibited similar $\sim 1 \mathrm{~nm}$ disordered grain boundaries containing approximately a monolayer of excess titanium and half that of iron in the films. ${ }^{207,208}$ Moreover, earlier TEM studies of quenched samples of less-pure $\mathrm{ZnO}-\mathrm{Bi}_{2} \mathrm{O}_{3}{ }^{209,210}$ and $\mathrm{TiO}_{2}$-rich $\mathrm{SrTiO}_{3}{ }^{211}$ revealed that each has a wetting transition for which $\psi \rightarrow 0$ above a critical temperature, $\sim 1150^{\circ}$ and $1450^{\circ} \mathrm{C}$, respectively. The transition temperatures were reduced with other impurities present for $\mathrm{SrTiO}_{3}{ }^{211}$ Rather similar results were recently seen in $\mathrm{Al}_{2} \mathrm{O}_{3}$ doped with $\mathrm{CaO}-\mathrm{SiO}_{2}$. The grain boundaries were wide disordered layers, yet contained only about a monolayer of adsorbed impurity, and the $\mathrm{Ca}$ :Si ratio was much higher than in the liquid pockets. ${ }^{171}$ Obviously, these results imply caution in interpreting earlier studies of oxides containing glassy impurities.

(B) Intergranular Films in $\mathrm{Si}_{3} \mathrm{~N}_{4}$ : (a) Overview: Numerous investigations of $\mathrm{Si}_{3} \mathrm{~N}_{4}$, have consistently revealed that a very high fraction of grain boundaries are composed of an amorphous silicate film that is considered to have an equilibrium thickness, and the film thickness depends as much or more on the composition of the system as on the crystallography of the boundary. ${ }^{155,180,181}$ Analytical TEM studies show that films often contain more nitrogen than do the pockets and can have different concentrations of cations. ${ }^{166-168}$ Moreover, for $\mathrm{Si}_{3} \mathrm{~N}_{4}$ grains equilibrated with a RE-aluminum silicate (where RE is one of the yttrium series or various rare earths), the mean film thickness depends on the specific rare earth used, but it is virtually the same for polycrystals with $5 \%-12 \%$ liquid by volume and for small clusters of grains found in dilute suspensions of powder in glass. ${ }^{179,181}$ Apparently, a particle-particle attraction exists in this system that is roughly comparable to the capillary forces often considered to drive sintering. However, in parallel, the $\alpha$ to $\beta$ transformation and subsequent particle growth (Ostwald ripening) occur via diffusion through the liquid and films. The rates are highly anisotropic, with preferred growth parallel to the $c$-axis, yielding long grains with strongly faceted prism faces. ${ }^{212}$

(b) In Situ Dispersion Force Determination by SR-VEELS: We have shown how to calculate full spectral Hamaker constants from experimental interband transition strengths. Quantitative analysis of SR-VEEL spectra and SR-VEEL spectrum images can now supply the required interband transition strength results for individual intergranular films (IGFs) in $\mathrm{Si}_{3} \mathrm{~N}_{4}$. The combination of SR-VEEL spectrum imaging and full spectral Hamaker constants permits the in situ determination of vdW forces on the IGFs in $\mathrm{Si}_{3} \mathrm{~N}_{4}$-based ceramics and on the local variations in dispersion forces throughout the microstructure of individual $\mathrm{Si}_{3} \mathrm{~N}_{4}$ samples. $^{24}$

The Hamaker constants representing the LD forces were determined from in situ SR-VEEL spectroscopy for three different IGF chemistries in $\mathrm{Si}_{3} \mathrm{~N}_{4}$ material. Full profiles from one $\mathrm{Si}_{3} \mathrm{~N}_{4}$ grain across the amorphous IGF into the grain on the other side were acquired with a new spectrum image acquisition system. The full data set was analyzed, and the interband transition strength determined as a function of beam position. From those data, the Hamaker constant was calculated for the actual interface investigated, based on spectra from the center of the IGF.

The samples studied fall into two classes: ${ }^{24}$ those based on lanthanide (R) glasses of the type R-Si-Al-O-N and on simple silicate glass compositions containing calcium of the type $\mathrm{Ca}-\mathrm{Si}$ $\mathrm{O}-\mathrm{N}$. The lanthanide glass samples used either yttrium-aluminum(YAl-) or lanthanum-aluminum- (LaAl-) doped silicon oxynitride (SiON) glass. ${ }^{212}$ The calcium-doped $\mathrm{SiON}$ glass- $\mathrm{Si}_{3} \mathrm{~N}_{4}$ samples were made by Tanaka. ${ }^{180,213}$ The oxygen content was $1.3 \mathrm{wt} \%$ and the calcium concentration was $450 \mathrm{ppm}$, with no other cation impurities detected.

SR-VEEL spectra were acquired on the $\mathrm{Si}_{3} \mathrm{~N}_{4}$ samples with a parallel EEL spectroscopy system fitted to a dedicated scanning transmission electron microscope (STEM) operated at $100 \mathrm{keV}$. The incident electron beam spot size was $0.6 \mathrm{~nm}$ diameter, and the energy resolution of the whole system was better than $0.7 \mathrm{eV}$. Each spectrum covered the energy-loss range from -20 to $80 \mathrm{eV}$. Figure 20 shows the microstructure of one of the interfaces analyzed in the $\mathrm{LaAl}-\mathrm{Si}_{3} \mathrm{~N}_{4}$ material.

The LD spectra $\varepsilon_{2}(\xi)$ were calculated and then accumulated in a spectral database from which any combinations of them could be used to calculate the Hamaker constants of interest. For each VEEL spectrum image, three interband transition strengths were selected, one from each $\mathrm{Si}_{3} \mathrm{~N}_{4}$ grain and one from the middle of the IGF (Fig. 21). Full spectral Hamaker constants for $A^{\mathrm{NR}}$ $\left[\mathrm{Si}_{3} \mathrm{~N}_{4} \mid\right.$ vacuum $\left.\mid \mathrm{Si}_{3} \mathrm{~N}_{4}\right]$ and $A^{\mathrm{NR}}\left[\mathrm{Si}_{3} \mathrm{~N}_{4}|\mathrm{IGF}| \mathrm{Si}_{3} \mathrm{~N}_{4}\right]$ were then determined from each SR-VEEL spectrum image in each type of $\mathrm{Si}_{3} \mathrm{~N}_{4}$ material.

At the end of the analysis of the SR-VEELS data, the index of refraction can be calculated from the dielectric function across the IGF (Fig. 22). The index for bulk $\mathrm{Si}_{3} \mathrm{~N}_{4}$ is recovered, but the index of refraction for the IGF also is obtained by this method.

The indexes of refraction for the IGFs and the Hamaker constants show a dependence on the composition of the IGF. When the Hamaker constant is plotted against the IGF index of refraction (Fig. 23), it is observed that the Hamaker constant decreases almost linearly with increasing index of refraction of the IGF.

From the profiles of the interband transition strength across the IGF, it is possible to measure an IGF thickness. This is not identical to the IGF thickness as determined by HREM but is related to it. Here it serves as an indication of the variation in IGF thickness between different interfaces in a particular material. A definite correlation between this IGF thickness and the Hamaker constant exists for the lanthanum-aluminum material (Fig. 24). 




Fig. 20. STEM micrographs of a $\mathrm{LaAl}-\mathrm{Si}_{3} \mathrm{~N}_{4}$ sample showing where SR-VEEL spectrum images were acquired. The $10 \mathrm{~nm}$ spectrum image (a line along which the multiplexed SR-VEEL spectrum image was acquired) is shown. (Reprinted with permission from Elsevier Science. ${ }^{24}$ )

The variation of the dispersion forces on the IGFs in these three types of $\mathrm{Si}_{3} \mathrm{~N}_{4}$ are shown in Fig. 25 and vary from 2 to $23 \mathrm{zJ}$, a factor of $>10$ in the Hamaker constant. Previously, there may have been speculation that variations in the dispersion force were not important in dictating the force balance. However, a variation by a factor of 10 in the dispersion force might well be an important component in the force balance in optimized $\mathrm{Si}_{3} \mathrm{~N}_{4}$ materials exhibiting equilibrium IGFs. If the dispersion force is the major attractive force counterbalancing the other repulsive terms, then changes of this magnitude in the attractive term are critical. Alternatively, the dispersion force variation might become negligible in comparison to other terms that are of larger absolute magnitude, with the effect that the dispersion force would not be important. However, the present results discount this idea. From the study of individual IGFs in $\mathrm{LaAl}-\mathrm{Si}_{3} \mathrm{~N}_{4}$, a direct correlation between variations in the dispersion force on the IGF and the measured IGF thickness is found. This demonstrates that for the

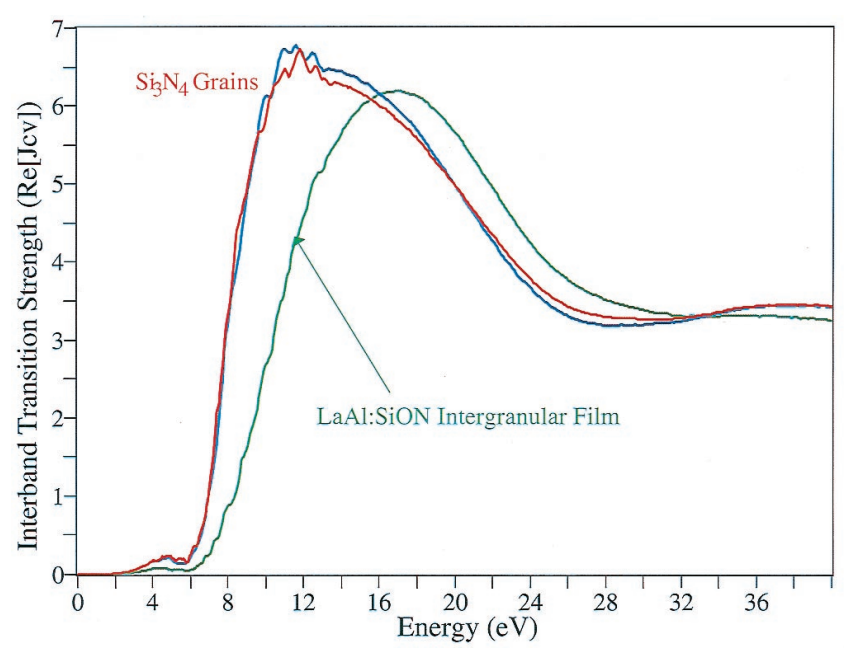

Fig. 21. Interband transition strength spectra for the IGF and each $\mathrm{Si}_{3} \mathrm{~N}_{4}$ grain from $\mathrm{LaAl}-\mathrm{Si}_{3} \mathrm{~N}_{4}$. (Reprinted with permission from Elsevier Science. ${ }^{24}$ )

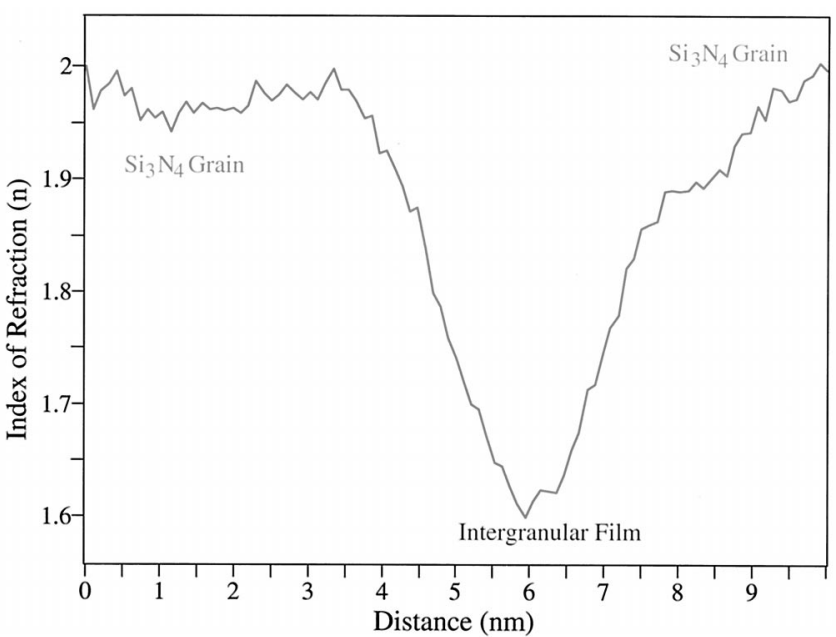

Fig. 22. Index of refraction determined from the index sum rule applied to the interband transition strength spectrum image from LaAl- $-\mathrm{Si}_{3} \mathrm{~N}_{4}$. (Reprinted with permission from Elsevier Science. ${ }^{24}$ )

optimized $\mathrm{Si}_{3} \mathrm{~N}_{4}$ materials studied here, which have been sintered to a final state microstructure and exhibit equilibrium IGFs, a major contributing term in the IGF thickness is the LD force on the IGFs. The IGF thicknesses in various optimized $\mathrm{Si}_{3} \mathrm{~N}_{4}$ samples with common glass systems correlate very closely to the magnitudes of the Hamaker constants of, and dispersion forces at, the IGFs. From HR-TEM measurements, the average IGF thicknesses for these materials are 1.2, 1.45, and $1.6 \mathrm{~nm}$ for YAl-, Ca-, and LaAl-doped $\mathrm{SiON}$ glass- $\mathrm{Si}_{3} \mathrm{~N}_{4}$ materials. Considering the lanthanide-doped glass system, the LaAl-doped system has lower values of the dispersion force for three of the four interfaces, and this material exhibits thicker intergranular films. At the same time, variations of the dispersion force within a single material are also apparent and can be correlated to the variation of the equilibrium film thicknesses from their average values for the material.

The SR-VEELS method of in situ Hamaker constant determination is accurate and reproducible with comparison to VUV measurements for the bulk materials and repeated measurements on numerous individual IGFs. For various glass additives having various film thickness, the accurate Hamaker constants provide a basis to calculate the first term in the controlling force balance (Eq. (53)). Local variations in Hamaker constants within the microstructure of a single sample correlate inversely to the distribution of IGF thickness observed. For these optimized $\mathrm{Si}_{3} \mathrm{~N}_{4}$ materials,

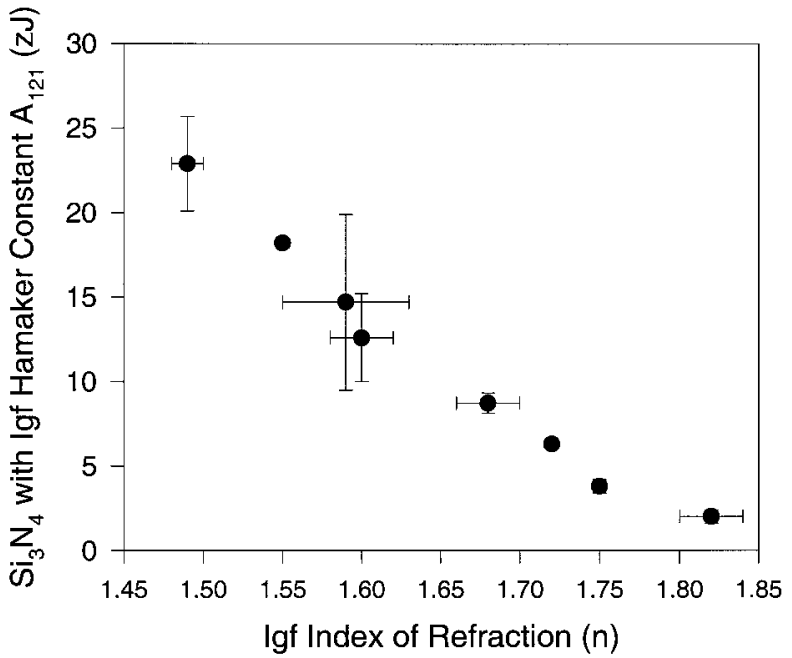

Fig. 23. Hamaker constant $A_{121}^{\mathrm{NR}}$ as a function of the index of refraction of the IGF for all the boundaries studied. (Reprinted with permission from Elsevier Science. ${ }^{24}$ ) 


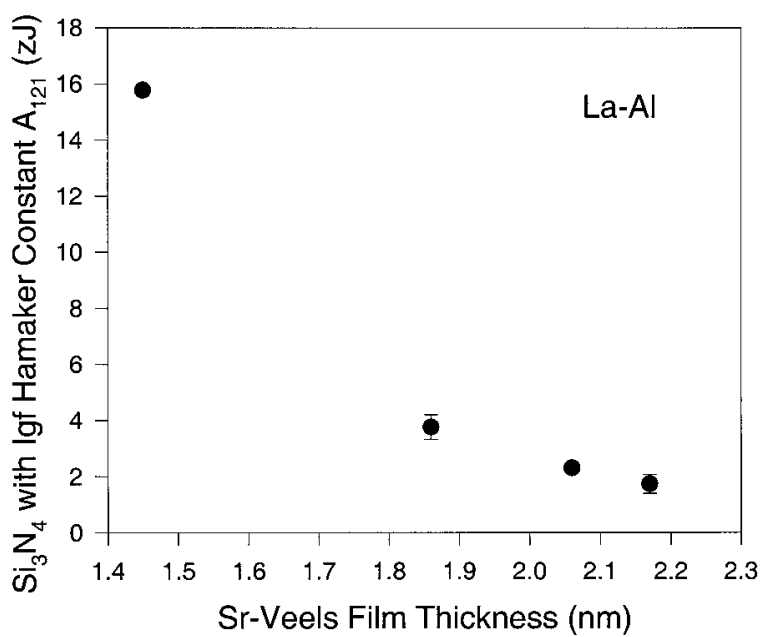

Fig. 24. Hamaker constant $A_{121}^{\mathrm{NR}}$ for $\mathrm{LaAl}-\mathrm{Si}_{3} \mathrm{~N}_{4}$ material as a function of the IGF thickness as measured from the SR-VEEL index of refraction profiles across the IGF.

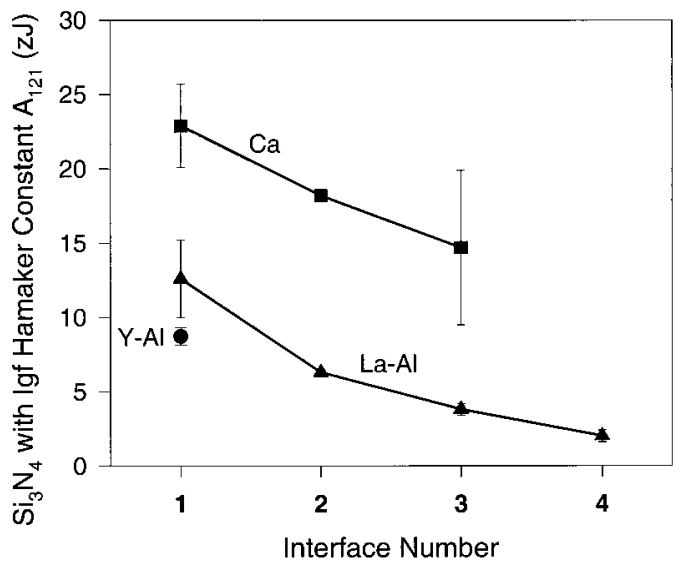

Fig. 25. Hamaker constant $A_{121}^{\mathrm{NR}}$ for $\left[\mathrm{Si}_{3} \mathrm{~N}_{4}|\mathrm{IGF}| \mathrm{Si}_{3} \mathrm{~N}_{4}\right]$ for each interface studied in the LaAl-, YAl-, and Ca-doped $\mathrm{SiON}$ glass- $\mathrm{Si}_{3} \mathrm{~N}_{4}$ materials.

the dispersion forces vary over a range from 2 to $12 \mathrm{zJ}$, corresponding to a $50 \%$ increase of the IGF thickness. This ability to experimentally determine Hamaker constants in situ represents an important new tool for dispersion force and wetting studies.

(C) Intergranular and Surficial Films in $\mathrm{ZnO}$ : The ability to rigorously calculate Hamaker constants for inorganic systems has facilitated the study of interfacial phenomena in many other ceramics. Chiang and co-workers ${ }^{170,214-216}$ have systematically studied the model varistor system $\mathrm{Bi}_{2} \mathrm{O}_{3}$-doped $\mathrm{ZnO}$, and they have determined that the equilibrium state of nonspecial grain boundaries is a disordered, bismuth-enriched film of 1-1.5 nm thickness (Fig. 26), not unlike the siliceous films found in other oxides and $\mathrm{Si}_{3} \mathrm{~N}_{4}$. Moreover, the bismuthate film coexists with bulk solid or liquid phases, and it has been argued to be a true surface phase whose thickness and composition are determined by dispersion forces among other interactions. ${ }^{215}$ These intergranular films have been implicated in subsolidus-activated sintering ${ }^{217}$ as well as varistor behavior. ${ }^{218}$

Recently, surface amorphous films of stable, nanometer thickness also have been identified in $\mathrm{Bi}_{2} \mathrm{O}_{3}-\mathrm{ZnO}$ (Fig. 27) and other oxides, ${ }^{219,220}$ the quantitative interpretation of which requires evaluation of the $A^{\mathrm{NR}}$ Hamaker constant for surface layers, a quantity that can be positive or negative, acting to thin or thicken a surface film.

(D) Diffuse Interface Theory: The properties of diffuse or graded interfaces are of general interest in ceramic systems. For the LD interaction, we have discussed the solutions for $\left(A_{123}^{\mathrm{NR}}\right.$ and
$A_{12321}^{\mathrm{NR}}$ ). As the interlayer becomes more complex, the interaction energy becomes dependent on the particulars of the structural variation of the interlayer. Systems more complex than $A_{12321}^{\mathrm{NR}}$ are often seen in biology, where the behavior of complex many-layer films result in cell walls and cell-cell interactions. Parseg$\operatorname{ian}^{43,221,222}$ has developed a solution for the LD interaction for multilayered interlayers, but only in the approximation that the center-most single layer has variable thickness. This is the same implicit approximation that was used for analysis of the soap film ${ }^{42}$ and may not have applicability in many ceramic systems.

Additional aspects of the interfacial properties of graded interfaces, beyond dispersion, have been discussed recently in the literature. For example Ackler et al. ${ }^{223}$ have investigated the effects of amorphous, thin intergranular film that abuts crystalline surfaces with differing orientation. They use a diffuse interface method to calculate the variation of order that is induced by the

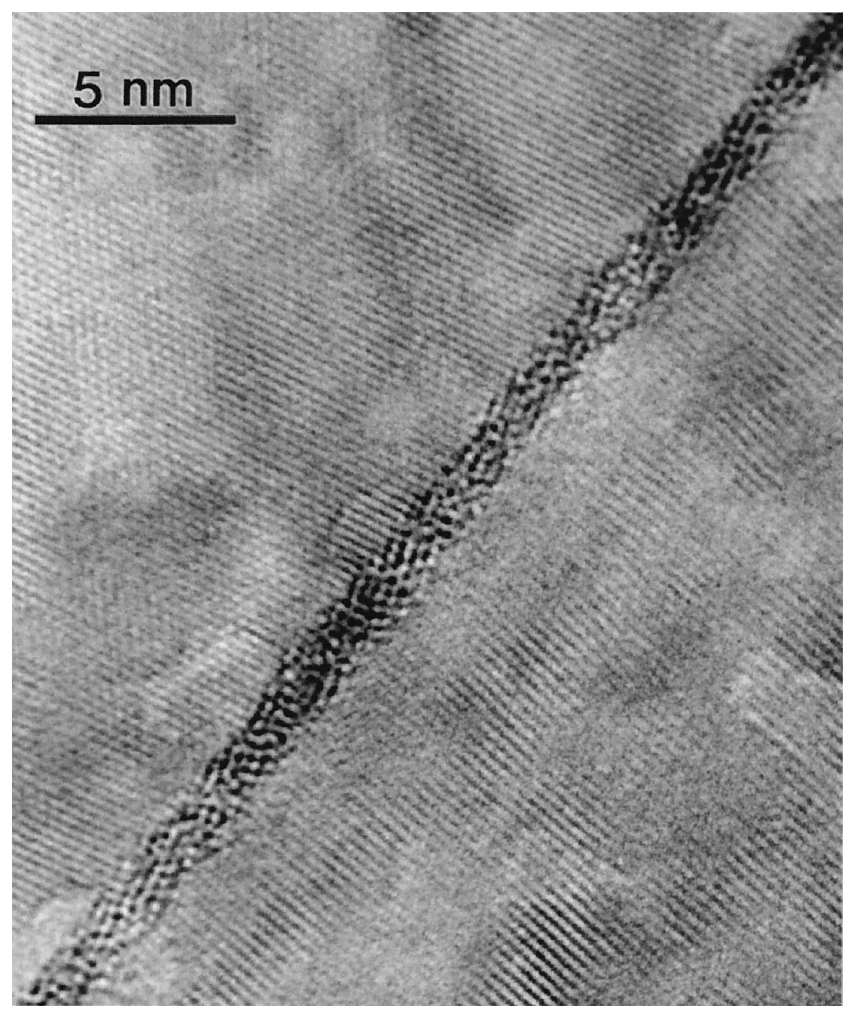

Fig. 26. Intergranular film, shown at a nonspecial grain boundary, in a model varistor system of $\mathrm{Bi}_{2} \mathrm{O}_{3}$-doped $\mathrm{ZnO}$. Interlayer film is a disordered, bismuth-enriched film of $1-1.5 \mathrm{~nm}$ thickness.

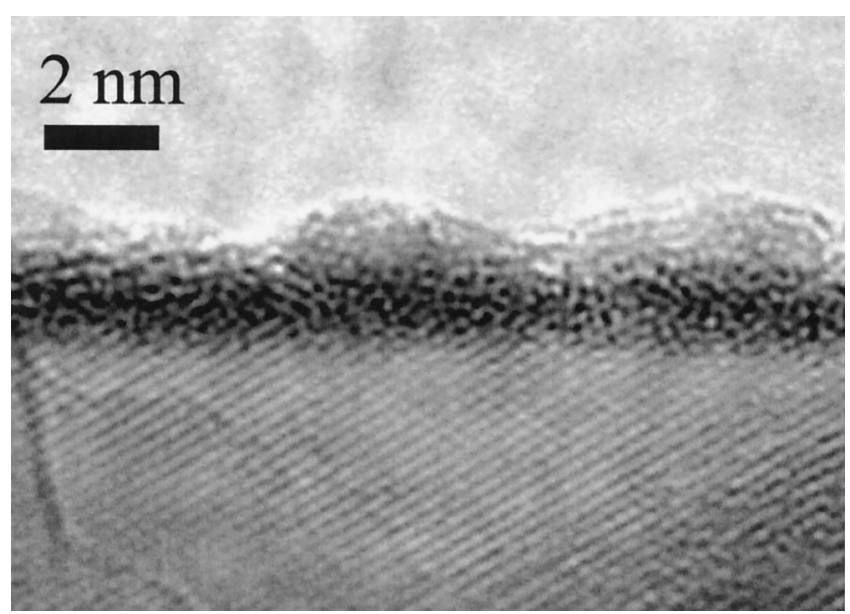

Fig. 27. Amorphous surficial film of stable nanometer scale thickness seen in $\mathrm{Bi}_{2} \mathrm{O}_{3}$-doped $\mathrm{ZnO}$. 
presence of each crystalline surface. Their results indicate that, depending on misorientation of the crystals, the induced order can produce positive or negative steric forces on the thin amorphous film. The theory depends on measurable bulk thermodynamic properties. Bobeth, Clarke, and Pompe ${ }^{224}$ have published a diffuse interface theory that treats composition gradients between triple junctions and adjoining thin-film regions. They calculate an effective osmotic force that accounts for observed variations in film thickness with solute addition. Warren, Carter, and Kobayashi $^{225}$ have formulated a diffuse interface theory that includes crystalline orientation and induced interfacial order as well as composition as order parameters. This method has been used to calculate microstructural evolution for systems of many crystallites growing in an amorphous phase. Thin intergranular films have been observed in these simulations but have not been characterized.

(E) When Is an IGF Too Thin-Continuum Approximation: An interesting question arises in the case of very thin intergranular films: When is the film thickness so thin that we are violating the continuum approximation inherent in the Lifshitz approach? Or, what is the smallest quantity of the material that has the properties that are essentially bulklike and that have comparable atomic structure, oxidation states, and electronic structure and bonding and, therefore, optical properties? Because the LD interaction is essentially electronic in nature, resulting from the interband transitions of the electronic structure, it is the nature of the bonding and its extent in real space that determines the intrinsic scaling that, in turn, determines the minimum size for the continuum approximation. Therefore, in vdW solids, such as polymeric materials, the interatomic bonding is fully satisfied in the polymer chain, and it is the size of the molecular moiety, such as the chain diameter or chain length, or a few times the chain diameter, that can be considered as the smallest length for which bulk, continuum-like, properties would be expected. For silicates and glass formers, this intrinsic length scale is given by the tetrahedra size of 0.23 or $0.15 \mathrm{~nm}$; therefore, the bulklike electronic structure for silicates should be observed for silicates in the range of a few $\mathrm{SiO}_{2}$ tetrahedra or $\sim 0.6 \mathrm{~nm}$ in size. ${ }^{226}$ For materials with extensive three-dimensional bonding, the intrinsic length necessary to establish the bulklike electronic structure may be larger. For particular materials of interest, this question is answered by comparing bulk band structure calculations with electronic structure calculations for clusters of increasing size. The cluster size necessary to realize a bulklike electronic structure represents the
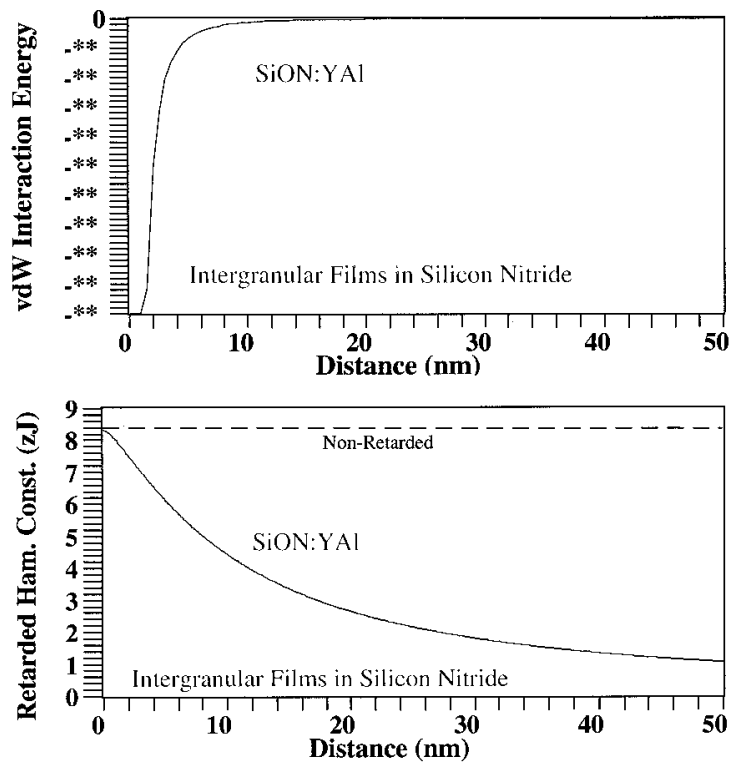

Fig. 28. YAl-SiON intergranular films in $\mathrm{Si}_{3} \mathrm{~N}_{4}$, showing normal retardation effects corresponding to a nonwetting condition, as shown in (a) the interaction energy (arbitrary units) and (b) the retarded Hamaker constant. size necessary to satisfy the continuum approximation of Lifshitz theory. It also can be determined by considering the real space extent of the individual electronic wave functions in the material, as can be determined from electronic structure calculations. ${ }^{227}$ The intrinsic length scale for the transition to continuum behavior for various materials is a topic that warrants additional study.

\section{(8) Retardation of Dispersion Forces}

(A) Normal Retardation Effects-Reduction in the Power-Law Dependence: If we consider the case of the intergranular films in $\mathrm{Si}_{3} \mathrm{~N}_{4}$ first discussed in Section $\operatorname{III}(7)(\mathrm{B})(\mathrm{b})$, we can calculate the $\mathrm{LD}$ or vdW interaction energy and the retarded Hamaker spectrum for $A_{121}^{\mathrm{R}}$ for material $1\left(\mathrm{Si}_{3} \mathrm{~N}_{4}\right)$ and material 2 (the YAl-SiON glass) shown in Fig. 28. The energy minimum at zero interlayer thickness shows that this is an attractive dispersion force. The nonretarded Hamaker constant is 8, but, with increasing interlayer thickness, the Hamaker constant monotonically decreases. At a film thickness of $10 \mathrm{~nm}$, the Hamaker constant has been decreased by a factor of 2. This case is an example of "normal retardation"; the outcome of the retardation is a decrease in the power-law dependence.

Let us consider an example of normal retardation in a surficial film. A surficial film of the same YAl-SiON glass on a $\mathrm{Si}_{3} \mathrm{~N}_{4}$ substrate gives an interaction energy, shown in Fig. 29(a), that reaches its minimum at infinite film thickness, representing a wetting film condition in which the film would, if able to approach equilibrium, approach infinite thickness. The Hamaker constant, shown in Fig. 29(b), is negative, demonstrating the repulsive force between the air-glass interface and the glass- $\mathrm{Si}_{3} \mathrm{~N}_{4}$ interface. As the surficial film thickens, the Hamaker constant decreases monotonically from a nonretarded value of $17 \mathrm{zJ}$ to $8 \mathrm{zJ}$ at $15 \mathrm{~nm}$.

(B) Equilibrium Surficial Films: The LD interaction can result in equilibrium surficial films in systems, such as water on ice, that exhibit an equilibrium film thickness, typically on the order of $4 \mathrm{~nm}$. The appearance of equilibrium surficial films is very different from the normal effects of LD forces on surficial films, where either a repulsive interaction produces wetting films or an attractive interaction produces dewetting films. To understand the occurrence of equilibrium thickness surficial films of, for example, water on ice, we need to understand the effect of retardation on the magnitude of the vdW dispersion forces and the direct effect of film thickness in modifying these forces because of the effects of retardation.

(a) Water on Ice: The direct cause of the equilibrium surficial films of water on ice seen at temperatures to $40^{\circ} \mathrm{C}$ below the


Fig. 29. YAl-SiON surficial films in $\mathrm{Si}_{3} \mathrm{~N}_{4}$, showing normal retardation effects corresponding to a nonwetting condition, as shown in (a) the interaction energy (arbitrary units) and (b) the retarded Hamaker constant. 
melting point is the change in the electronic structure of $\mathrm{H}_{2} \mathrm{O}$ between the liquid and solid states. Consider the interband transitions of water and ice shown in Fig. 30. ${ }^{87,88}$ Although the bandgaps of water $(n=1.41)$ and ice $(n=1.31)$ are comparable, there are some differences in the interatomic bonding in the region of $10-16 \mathrm{eV}$, and a dramatic difference in the region of $24-50 \mathrm{eV}$. The large set of transitions in ice results from bonding between the $\mathrm{H}_{2} \mathrm{O}$ molecules in the solid, crystalline state. The introduction of these interatomic bonds at high energy leads to a strong attractive dispersion force at close approach, but, as the surficial film thickness increases, these high-energy bonds are the first to dephase. Another interesting spectral behavior is shown in Fig. 31, where the LD spectra of water and ice cross in the vicinity of 10.5 $\mathrm{eV}$. This spectral crossing in the dispersion spectra is the signature for equilibrium surficial film formation. Calculating the retarded dispersion interaction (Fig. 32) shows that the interaction energy does not exhibit the monotonic variation of the examples of normal retardation, but, rather, there is an energy minimum for the system at $3 \mathrm{~nm}$. The retarded Hamaker spectrum is also not monotonic, but, instead, has a zero crossing from negative, repulsive forces at close approach to positive, attractive forces at larger film thicknesses. Therefore, for film thicknesses $<3 \mathrm{~nm}$, the dispersion force tends to thicken the film, whereas, for film thicknesses $>3 \mathrm{~nm}$, the film tends to thin under the attractive dispersion forces.

(b) Lorentz Oscillator Doping for Equilibrium Surficial Films: The high-energy transitions of the substrate produce the LD spectrum crossing, thereby causing the formation of equilibrium surficial film formation for water on ice. A more practical method of designing materials with surficial films is to dope the wetting film material, leaving the substrate unchanged. ${ }^{228}$ Figure 33 shows the interband transitions for an $\mathrm{Al}_{2} \mathrm{O}_{3}$ substrate and a wetting film of $\mathrm{SiO}_{2}$. From the perspective of the LD interaction and considering the index of refraction, $\mathrm{SiO}_{2}(n=1.51)$ wets $\mathrm{Al}_{2} \mathrm{O}_{3}(n=$ 1.75). Using a Lorentz oscillator (LO) centered at $12 \mathrm{eV}$, we can add a set of interatomic bonds to the interband transition strength of the $\mathrm{SiO}_{2}$, synthesizing a new interband transition strength spectra comparable to a doped silicate glass with an increased index of refraction of 1.78. The LD spectra (shown in Fig. 34) for the LO-doped $\mathrm{SiO}_{2}$ now crosses the spectrum of $\mathrm{Al}_{2} \mathrm{O}_{3}$. From the index of refraction approximations, the LO-doped $\mathrm{SiO}_{2}$, whose index is higher than that of $\mathrm{Al}_{2} \mathrm{O}_{3}$, does not wet the $\mathrm{Al}_{2} \mathrm{O}_{3}$ substrate. However, from the calculation of the retarded Hamaker interaction, Figure 35 shows that there again is an energy minimum observed at $10 \mathrm{~nm}$ and a zero crossing of the Hamaker spectrum. This approach of synthesizing new LO-doped interband transition strengths permits the systematic study of the dispersion interactions and their use in materials design.

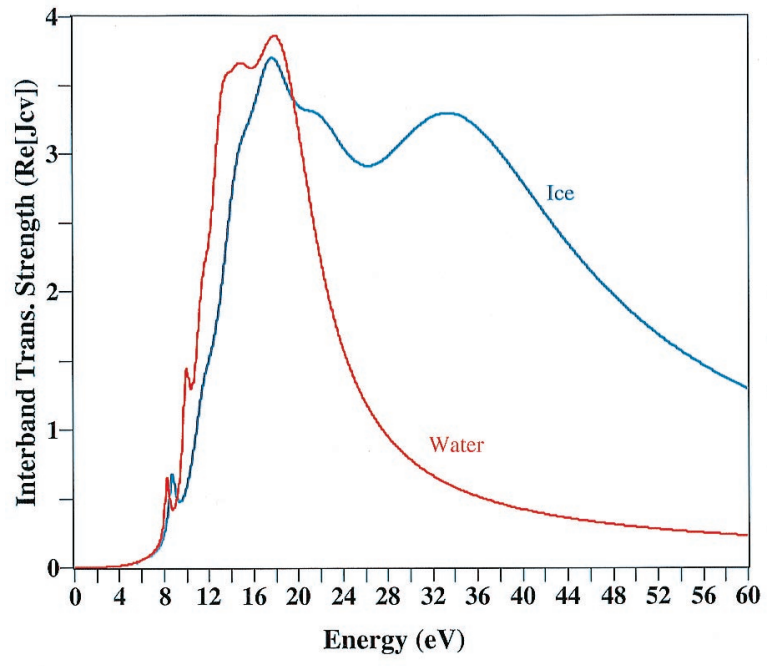

Fig. 30. Interband transition strength of (blue) ice and (red) water, showing large changes in the interatomic bonding in the frozen state.

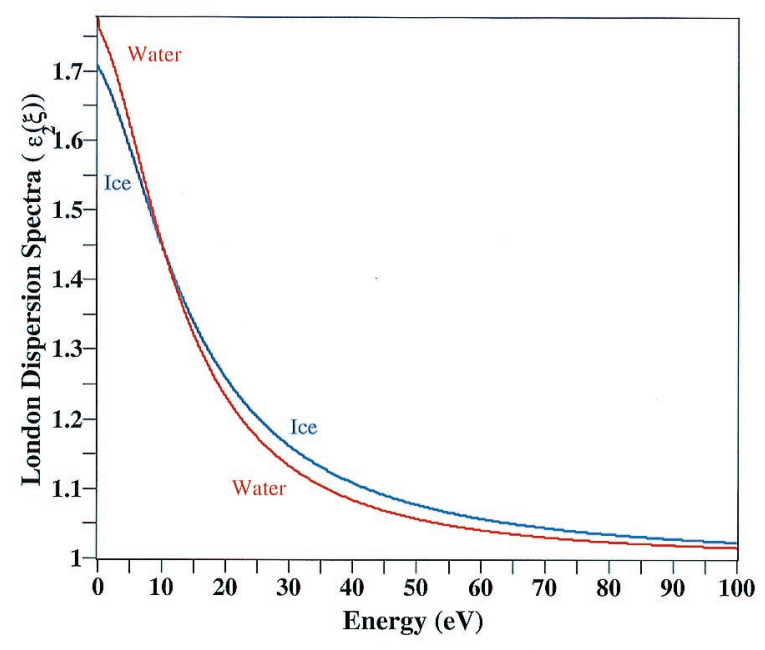

Fig. 31. LD spectra for (blue) ice and (red) water, showing a crossing of the LD spectra at $12 \mathrm{eV}$.

We have considered only the effects of dispersion; the behavior of any particular material may be dominated by other interactions. For other systems, such as hydrocarbon solvents wetting vdW solids, the dispersion interaction would be expected to dominate. $^{229}$

(C) Bimodal Wetting/Dewetting: The retardation of the LD interaction can result in other novel wetting conditions in addition to the equilibrium surficial films just discussed. Calculating the retarded Hamaker interaction of the $\mathrm{LaAl}-\mathrm{SiON}$ intergranular glass (discussed in Section $\operatorname{III}(7)(B)(b)$ ) as a surficial film on a $\mathrm{Si}_{3} \mathrm{~N}_{4}$ substrate produces a global energy maximum in the interaction energy shown in Fig. 36(a). This system either wets or dewets, depending on its initial conditions. If the initial film thickness is $<8 \mathrm{~nm}$, then the dispersion interaction is attractive, and the film thins. However, if the film thickness is $>8 \mathrm{~nm}$, then the interaction is repulsive and the film thickens. The retarded Hamaker spectrum shows the opposite curvature, compared with the equilibrium surficial film case, and also exhibits a zero crossing, with the transition changing from an attractive close approach to repulsive for thicker films. This condition corresponds to a bimodal wetting/dewetting condition, an interesting film instability with potentially useful applications.
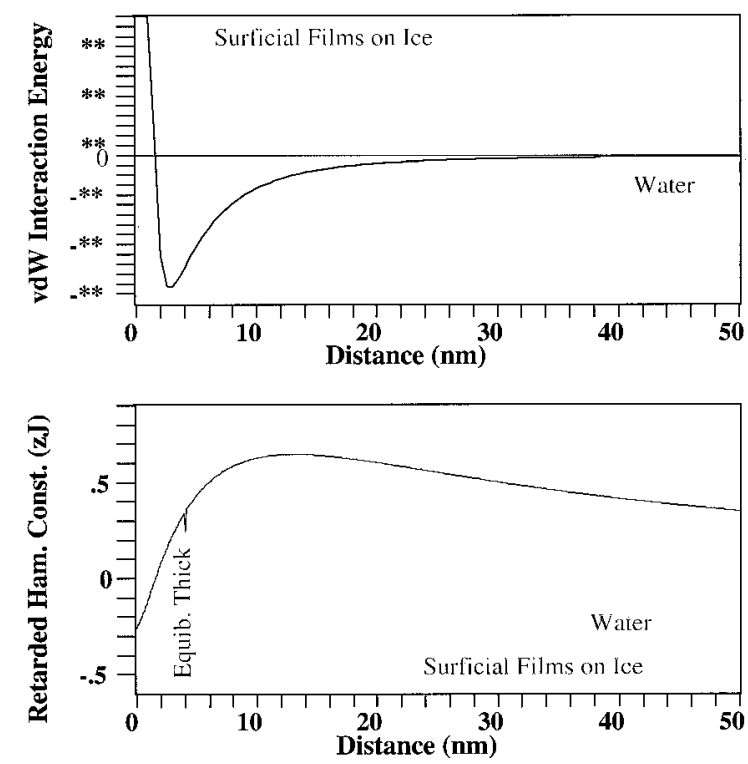

Fig. 32. Water on ice, showing the appearance of an equilibrium surficial film, as shown in (a) the interaction energy (arbitrary units) and (b) the retarded Hamaker constant. 


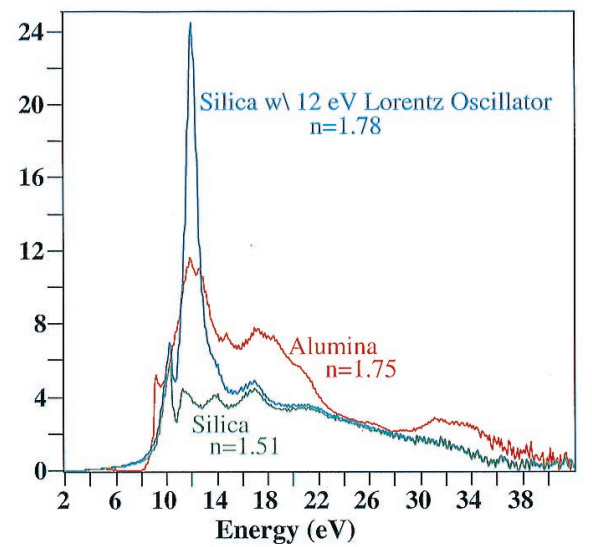

Fig. 33. Interband transition strength of (red) $\mathrm{Al}_{2} \mathrm{O}_{3}$ and (green) $\mathrm{SiO}_{2}$ along with a (blue) synthetic spectra of $\mathrm{SiO}_{2}$ doped with the addition of a $\mathrm{LO}$ to represent interatomic bonds at $12 \mathrm{eV}$.

\section{Conclusions}

The London dispersion (LD) forces, along with the Debye and Keesom forces, constitute the long-range van der Waals forces. London and Hamaker's work on the point-to-point dispersion interaction and Lifshitz's development of the continuum theory of dispersion are the foundations of our understanding of dispersion forces. Dispersion forces are present for all materials and are intrinsically related to the optical properties and the underlying interband electronic structure of materials. The force law scaling constant of the dispersion force, known as the Hamaker constant, can be determined from spectral or parametric optical properties of materials combined with knowledge of the configuration of the materials. With recent access to new experimental and ab initio tools for determination of optical properties of materials, dispersion force research has new opportunities for detailed studies. There are three opportunities in dispersion force research. The first is the development of improved index approximations that more accurately represent nonvacuum interlayers. The second is the development of Kramers-Kronig consistent parametric representations of the optical properties that enables improved estimation of Hamaker constants. Expanded databases of LD spectra of materials permit accurate estimation of nonretarded and retarded dispersion forces in complex configurations. The third is the development of solutions for generalized multilayer configurations of materials that are needed for the treatment of more-complex problems, such as graded interfaces.

Dispersion forces are critical in materials applications. Typically, they are a component with other forces in a force balance, and it is this balance that dictates the resulting behavior. The ubiquitous nature of the LD forces makes them a factor in a wide

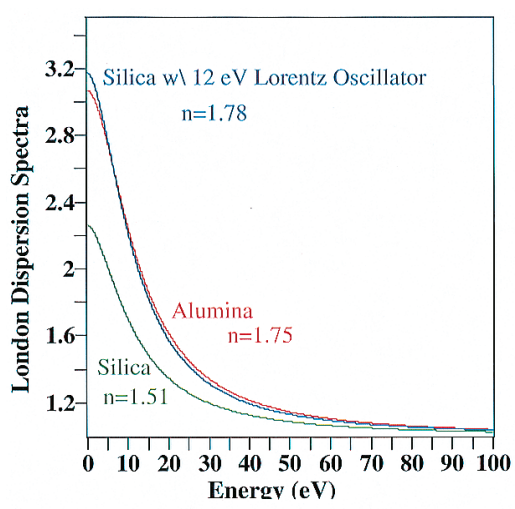

Fig. 34. LD spectra for the (red) $\mathrm{Al}_{2} \mathrm{O}_{3}$, (green) $\mathrm{SiO}_{2}$, and (blue) $\mathrm{SiO}_{2}$ with the $12 \mathrm{eV} \mathrm{LO}$ dopant. LD spectra crosses the doped and undoped $\mathrm{SiO}_{2}$ near $10 \mathrm{eV}$.
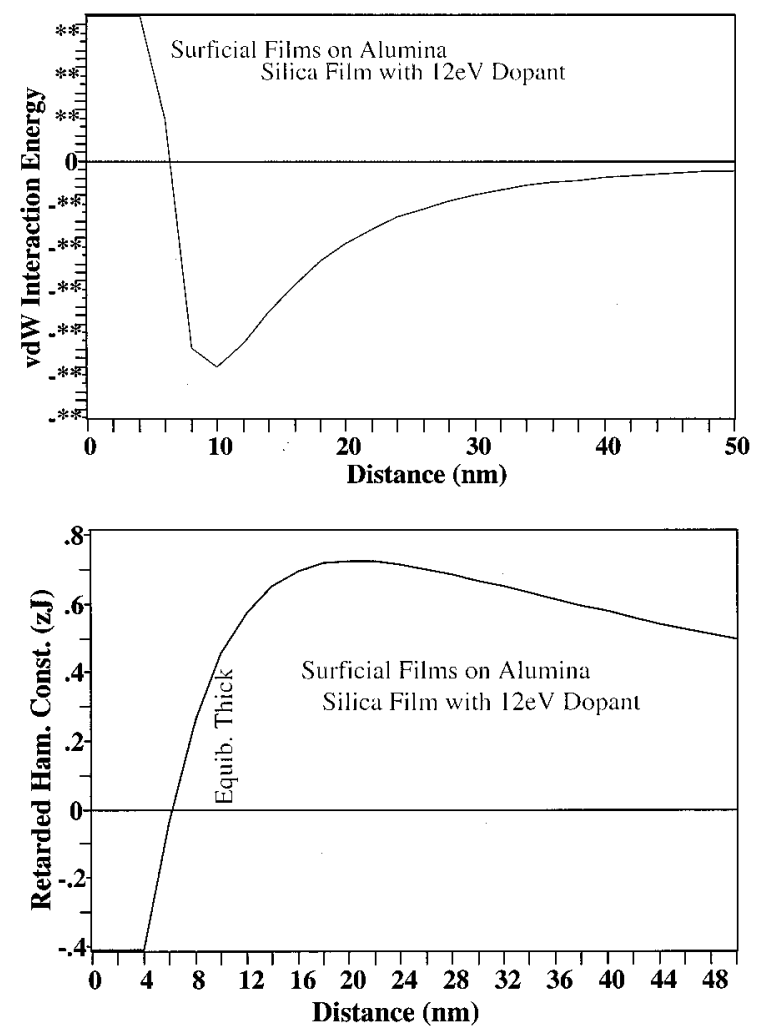

Fig. 35. LO doping of a $\mathrm{SiO}_{2}$ interlayer material showing the appearance of an equilibrium surficial film, as shown in (a) the interaction energy (arbitrary units) and (b) the retarded Hamaker constant.

spectrum of problems; they have been in evidence since the pioneering work of Young and Laplace on wetting, contact angles, and surface energies. Additional applications include the interparticle forces that can be measured by direct techniques, such as atomic force microscopy. LD forces are important in adhesion and in sintering, where the detailed shape at the crack tip and at the sintering neck can be controlled by the dispersion forces. Dispersion forces have an important role in the properties of many ceramics that contain intergranular films, and here the opportunity exists for the development of an integrated understanding of
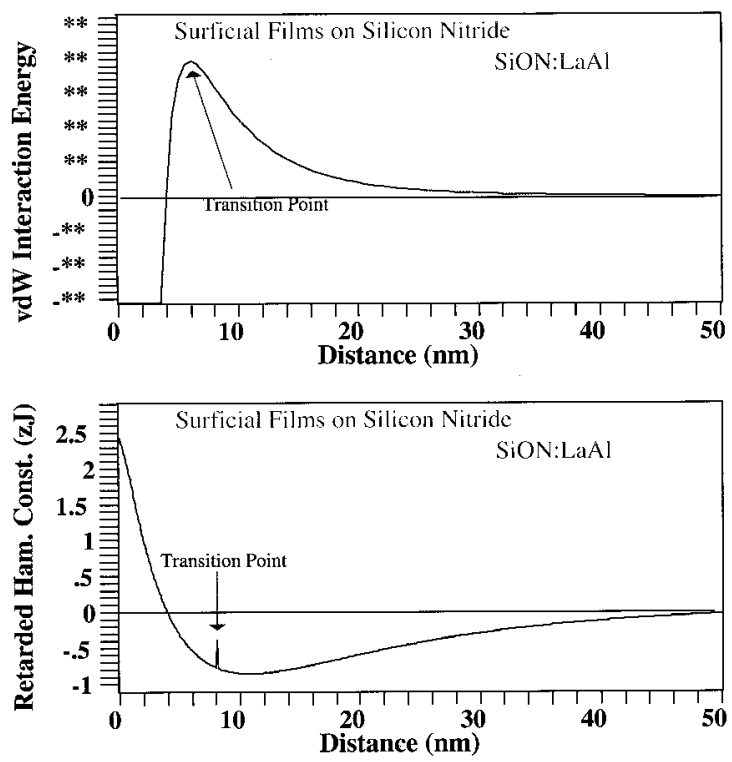

Fig. 36. Surficial film of LaAl-SiON glass on $\mathrm{Si}_{3} \mathrm{~N}_{4}$ substrate showing bimodal wetting/dewetting, as shown in (a) the interaction energy (arbitrary units) and (b) the retarded Hamaker constant. 
intergranular films that encompasses dispersion forces, segregation, multilayer adsorption, and structure. The intrinsic length scale at which there is a transition from the continuum perspective (dispersion forces) to the atomistic perspective (encompassing interatomic bonds) is critical in many materials problems, and the relationship of dispersion forces and intergranular films may represent an important opportunity to probe this topic.

The LD force is retarded at large separations, where the transit time of the electromagnetic interaction must be considered explicitly. Novel phenomena, such as equilibrium surficial films and bimodal wetting/dewetting, can occur in materials systems when the characteristic wavelengths of the interatomic bonds and the physical interlayer thicknesses lead to a change in the sign of the dispersion force. Use of these novel phenomena in future materials applications raises interesting opportunities in materials design.

\section{Acknowledgments}

The author would like to acknowledge the contributions of Anand Jagota to Section III(6), Rowland Cannon to Section III(7)(A), Yet-Ming Chiang to Section II(7)(C), W. Craig Carter to Section III(7)(D), and Barbara S. Brown for help preparing the manuscript. The author appreciates the long-standing support of the DuPont Co. The support and collaboration of Manfred Rühle and my Max-PlanckInstitut für Metallforschung collaborators, Harald Muellejans, Albert D. Dorneich, Gerd Duscher, Wilfried Sigle, Christina Scheu, Michael J. Hoffman, and Klaus van Benthem, are gratefully acknowledged. In addition, the collaborations with David J. Jones, Michael F. Lemon, Stephen Loughin, Lin K. DeNoyer, Rowland Cannon, Wai-Yim Ching, and Harold Ackler are also gratefully acknowledged. I acknowledge David R. Clarke who first encouraged my work in this field and helpful discussions with John Wettlaufer. The comments of the reviewers are also appreciated.

\section{References}

1(a)B. Taylor, "Concerning the Ascent of Water between Two Glass Plates," Philos. Trans. R. Soc. London, 27 [538.c.xx] 131 (1712). (b)F. Hauksbee, "Account of the Experiment on the Ascent of Water between Two Glass Plates, in a Hyperbolic Figure," Philos. Trans. R. Soc. London, 27 [539.c.xiv] 131 (1712). (Both of these papers are reproduced in the preface of R. Finn, Equilibrium Capillary Surfaces. Springer-Verlag, New York, 1986.)

T. Young, "An Essay on the Cohesion of Fluids," Philos. Trans. R. Soc. London, 95, 65-87 (1805).

${ }^{3}$ P. R. Pujado, H. Chun, and L. E. Scriven, "On the Attribution of an Equation of Capillarity to Young and Laplace," J. Colloid Interface Sci., 38, 662-63 (1972).

${ }^{4}$ J. Thomson, "On Certain Curious Motions Observable at the Surfaces of Wine and Other Alcoholic Liquors," Philos. Mag., 10, 330-33 (1855).

${ }^{5}$ J. D. van der Waals, "The Thermodynamic Theory of Capillarity Under the Hypothesis of a Continuous Variation of Density" (in Dutch), Verhandel. Konink. Akad. Weten. Amsterdam, 1, 8 (1893); translation published by J. S. Rowlinson, J. Statistical Phys., 20, 200-44 (1979).

${ }^{6} \mathrm{~J}$. W. Gibbs, Trans., "On the Equilibrium of Heterogeneous Substances," Connecticut Academy Transactions, 3, 108-248, 343-524 (1875-1877).

${ }^{7}$ J. W. Gibbs, The Scientific Papers of J. Willard Gibbs: Thermodynamics. Ox Bow Press, 1993.

${ }^{8}$ F. London, "The General Theory of Molecular Forces," Trans. Faraday Soc., 33, 8-26 (1937).

${ }^{9}$ F. London, "Zur Theorie und Systematik der Molekularkräfte," Z. Phys., 63, 245-79 (1930).

${ }^{10}$ Von R. Eisenschitz and F. London, "Über das Verhältnis der van der Waalsschen Kräfte zu den Homöopolaren Bindungskräften,” Z. Phys., 60, 491-527 (1930).

${ }^{11}$ F. London, "Über einige Eigenschaften und Anwendungen der Molekularkräfte," Z. Phys. Chem., B11, 222-51 (1930).

${ }^{12}$ H. C. Hamaker, "The London-van der Waals Attraction between Spherical Particles," Physica, 4 [10] 1058-72 (1937).

${ }^{13}$ W. Sellmeier, "II. Regarding the Sympathetic Oscillations Excited in Particles by Oscillations of the Ether and Their Feedback to the Latter, Particularly as a Means of Explaining Dispersion and its Anomalies" (in Ger.), Ann. Phys. Chem., 147, 525-54 (1872).

${ }^{14}$ See, for example, B. J. Hunt, The Maxwellians, Cornell History of Science. Cornell University Press, Ithaca, NY, 1994.

${ }^{15}$ P. J. Hahn, Oliver Heaviside: Sage in Solitude: The Life, Work, and Times of an Electrical Genius of the Victorian Age. IEEE, New York, 1988

${ }^{16}$ H. B. G. Casimir, "On The Attraction of Two Perfectly Conducting Plates," Proc. Koninklijke Nederlandse Akademie Van Wetenschappen, 51, 793-95 (1948).

${ }^{17}$ S. S. Schweber, QED and the Men Who Made It: Dyson, Freeman, Schwinger, and Tomonaga, Princeton Series in Physics. Princeton University Press, Princeton, NJ, 1994.

${ }^{18}$ (a)S. K. Lamoreaux, "Demonstration of the Casimir Force in the 0.6 to 6 Micron Range," Phys. Rev. Lett., 78, 5-8 (1997). (b)S. K. Lamoreaux, "Erratum: Demonstration of the Casimir Force in the 0.6 to 6 Micron Range," Phys. Rev. Lett., 81, 5475-76 (1998)

${ }^{19}$ E. M. Lifshitz, "The Theory of Molecular Attractive Forces between Solids," Sov. Phys. JETP, 2, 73-83 (1956).

${ }^{20}$ I. E. Dzyaloshinskii, E. M. Lifshitz, and L. P. Pitaevskii, "van der Waals Forces in Liquid Films," Sov. Phys. JETP, 37, [10] 161-70 (1960).
${ }^{21}$ I. E. Dzyaloshinskii, E. M. Lifshitz, and L. P. Pitaevskii “The General Theory of van der Waals Forces," Adv. Phys., 10 [38] 165-209 (1961)

${ }^{22}$ B. V. Derjaguin, A. S. Titijevskaia, I. I. Abricossova, and A. D. Malinka, "Investigations of the Forces of Interaction of Surfaces in Different Media and Their Application to the Problem of Colloid Stability," Discuss. Faraday Soc., 18, 25-41 (1954).

${ }^{23}$ R. H. French, R. M. Cannon, L. K. DeNoyer, and Y.-M. Chiang, "Full Spectral Calculation of Non-Retarded Hamaker Constants for Ceramic Systems from Interband Transition Strengths," Solid State Ionics, 75, 13-33 (1995).

${ }^{24}$ R. H. French, H. Müllejans, D. J. Jones, G. Duscher, R. M. Cannon, and M. Rühle, "Dispersion Forces and Hamaker Constants for Intergranular Film in Silicon Nitride from Spatially Resolved Valence Electron Energy Loss Spectrum Imaging," Acta Mater., 46 [7] 2271-87 (1998).

${ }^{25}$ V. A. Parsegian, "Long Range van der Waals Forces"; pp. 27-72 in Physical Chemistry: Enriching Topics from Colloid and Surface Science. Edited by H. van Olphen and K. J. Mysels. Theorex, La Jolla, CA, 1975.

${ }^{26}$ H. Margenau and J. Stamper, "Nonadditivity of Intermolecular Forces," Adv. Quantum Chem., 3, 129-60 (1967).

${ }^{27}$ B. Chu, Molecular Forces. Interscience, New York, 1967.

${ }^{28} \mathrm{H}$. Margenau and N. R. Kestner, Theory of Intermolecular Forces. Pergamon, New York, 1969.

${ }^{29}$ J. N Israelachvili, Intermolecular and Surface Forces, 2nd ed. Academic Press, London, U.K., 1992.

${ }^{30} \mathrm{~L}$. Bergström, "Hamaker Constants of Inorganic Materials," Adv. Colloid Interface Sci., 70, 125-69 (1997).

${ }^{31}$ D. Langbein, Theory of van der Waals Attraction, Springer Tracts in Modern Physics, Vol. 72; p. 139. Springer-Verlag, Berlin, Germany, 1974.

${ }^{32}$ J. W. Mahanty and B. W. Ninham, Dispersion Forces. Academic Press, New York, 1975.

${ }^{33}$ E. J. W. Verwey and J. Th. G. Overbeek, Theory of the Stability of Lyophobic Colloids. Elsevier, Amsterdam, The Netherlands, 1948

${ }^{34} \mathrm{D}$. J. Shaw, Introduction to Colloid and Surface Chemistry. ButterworthHeinemann, Oxford, U.K., 1993.

${ }^{35}$ W. H. Keesom, "On the Deduction of the Equation of State from Boltzmann's Entropy Principle"; pp. 3-20 in Communications Physical Laboratory, University of Leiden Supplement 24a to No. 121-132. Edited by H. Kamerlingh Onnes. Eduard Ijdo, Leyden, The Netherlands, 1912

${ }^{36}$ W. H. Keesom, "On the Deduction from Boltzmann's Entropy Principle of the Second Virial Coefficient for Material Particles (in the Limit Rigid Spheres of Central Symmetry) which Exert Central Forces Upon Each Other and for Rigid Spheres of Central Symmetry Containing an Electric Doublet at Their Centers"; pp. 23-41 in Communications Physical Laboratory, University of Leiden Supplement 24b to No. 121-132. Edited by H. Kamerlingh Onnes. Eduard Ijdo, Leyden, The Netherlands, 1912

${ }^{37}$ W. H. Keesom, "On the Second Virial Coefficient for Di-atomic Gases"; pp. 3-19 in Communications Physical Laboratory, University of Leiden Supplement 25 to No. 121-132. Edited by H. Kamerlingh Onnes. Eduard Ijdo, Leyden, The Netherlands, 1912

${ }^{38}$ W. H. Keesom, "On the Second Virial Coefficient for Monatomic Gases and for Hydrogen Below the Boyle-Point"; see Ref. 36, pp. 3-9.

${ }^{39}$ P. J. W. Debye, "Die van der Waalsschen Kohäsionskräfte," Phys. Z., 21, $178-87$ (1920).

${ }^{40}$ P. J. W. Debye, "Molekularkräfte und ihre Elektrische Deutung," Phys. Z., 22, 302-308 (1921)

${ }^{41}$ J. H. De Boer, "The Influence of van der Waals' Forces on Primary Bonds on Binding Energy, Strength, and Orientation, with Special Reference to Some Artificial Resins," Trans. Faraday Soc., 32, 10-38 (1936).

${ }^{42}$ B. W. Ninham and V. A. Parsegian, "van der Waals Forces Across Triple-Layer Films," J. Chem. Phys., 52, 4578-87 (1970).

${ }^{43}$ V. A. Parsegian and B. W. Ninham, "van der Waals Forces in Many-Layered Structures: Generalizations of the Lifshitz Result for Two Semi-infinite Media," $J$. Theor. Biol., 38, 101-109 (1973).

${ }^{44}$ V. A. Parsegian and B. W. Ninham, "Temperature-Dependent van der Waals Forces," Biophys. J., 10, 664-74 (1970).

${ }^{45}$ L. Landau and E. M. Lifshitz, Electrodynamics of Continuous Media. Addison Wesley, Reading, MA, 1960

${ }^{46}$ A. A. Abrikosov, L. P. Gorkov, and I. E. Dzyaloshinskii, Methods of Quantum Field Theory in Statistical Physics. Prentice-Hall, Englewood Cliffs, NJ, 1963.

${ }^{47}$ H. B. G. Casimir and D. Polder, "The Influence of Retardation on the London-van der Waals Forces," Phys. Rev. B, 73, 360-72 (1948).

${ }^{48}$ H. Wennerström, J. Daicic, and B. W. Ninham, "Temperature Dependence of Atom-Atom Interactions," Phys. Rev. A: Gen. Phys., 60, 2581-84 (1999).

${ }^{49}$ W. Black, J. G. V. De Jongh, J. Th. G. Overbeek, and M. J. Sparnay, "Measurements of Retarded van der Waals Forces," Trans. Faraday Soc., 56, 1597-608 (1960).

${ }^{50}$ M. Elbaum and M. Schick, "Application of the Theory of Dispersion Forces to the Surface Melting of Ice," Phys. Rev. Lett., 66, 1713-16 (1991).

${ }^{51}$ L. A. Wilen, J. S. Wettlaufer, M. Elbaum, and M. Schick, "Dispersion-Force Effects in Interfacial Premelting of Ice," Phys. Rev. B: Conds, Matter, 52, 12426-33 (1995).

${ }^{52}$ J. S. Wettlaufer and J. G. Dash, "Melting Below Zero," Sci. Am., 282 [2] 50-53 (2000)

${ }^{53}$ R. H. French, "Electronic Structure of $\alpha-\mathrm{Al}_{2} \mathrm{O}_{3}$, with Comparison to AlON and AlN," J. Am. Ceram. Soc., 73 [3] 477-89 (1990).

${ }^{54}$ R. H. French, D. J. Jones, and S. Loughin, "Interband Electronic Structure of $\alpha-\mathrm{Al}_{2} \mathrm{O}_{3}$ up to $2167 \mathrm{~K}, "$ J. Am. Ceram. Soc., 77, 412-22 (1994).

${ }^{55}$ R. H. French, S. J. Glass, F. S. Ohuchi, Y.-N Xu, F. Zandiehnadem, and W. Y. Ching, "Experimental and Theoretical Studies on the Electronic Structure and Optical Properties of Three Phases of $\mathrm{ZrO}_{2}$," Phys. Rev. B: Conds. Matter, 49, 5133-42 (1994). 
${ }^{56}$ W. Y. Ching, "Theoretical Studies of the Electronic Properties of Ceramic Materials," J. Am. Ceram. Soc., 73, 3135-60 (1990).

${ }^{57}$ S. Loughin, R. H. French, W. Y. Ching, Y. N. Xu, and G. A. Slack, "Electronic Structure of Aluminum Nitride: Theory and Experiment," Appl. Phys. Lett., 63, 1182-84 (1993)

${ }^{58}$ F. Wooten, Optical Properties of Solids; p. 49. Academic Press, New York, 1972.

${ }^{59} \mathrm{P}$. J. Mohr and B. N. Taylor, "CODATA Recommended Values of the Fundamental Physical Constants: 1998," National Institute of Standards and Technology, Gaithersburg, MD (http://physics.nist.gov/constants).

${ }^{60}$ R. H. French, "Laser-Plasma-Sourced, Temperature-Dependent VUV Spectrophotometer Using Dispersive Analysis," Phys. Scr., 41 [4] 404-408 (1990).

${ }^{61}$ M. L. Bortz and R. H. French, "Optical Reflectivity Measurements Using a Laser Plasma Light Source," Appl. Phys. Lett., 55 [19] 1955-57 (1989).

${ }^{62}$ R. H. French, H. Müllejans, and D. J. Jones, "Optical Properties of Aluminum Oxide: Determined from Vacuum Ultraviolet and Electron Energy Loss Spectroscopies," J. Am. Ceram. Soc., 81 [10] 2549-57 (1998).

${ }^{63}$ S. Loughin and R. H. French, "Aluminum Nitride (AlN)"; pp. 373-401 in Handbook of Optical Constants of Solids, Vol. III. Edited by E. Palik. Academic Press, New York, 1998.

${ }^{64}$ R. H. French, D. J. Jones, H. Müllejans, S. Loughin, A. D. Dorneich, and P. F. Carcia, "Optical Properties of Aluminum Nitride: Determined from Vacuum Ultraviolet Spectroscopy and Spectroscopic Ellipsometry," J. Mater. Res., 14, 4337-44 (1999).

${ }^{65}$ B. Johs, R. H. French, F. D. Kalk, W. A. McGahan, and J. A. Woollam, "Optical Analysis of Complex Multilayer Structures Using Multiple Data Types;" pp. 1098-106 in Optical Interference Coatings, Vol. 2253. SPIE, Bellingham, WA, 1994.

${ }^{66}$ R. de L. Kronig, "On the Theory of Dispersion of X-rays," J. Opt. Soc. Am., 12, 547-57 (1926).

${ }^{67}$ C. J. Gorter and R. de L. Kronig, "On the Theory of Absorption and Dispersion in Paramagnetic and Dielectric Media," Physica III, 9, 1009-20 (1936).

${ }^{68}$ H. A. Kramers, "La Diffusion de la Lumière par les Atomes," Atti. Congr. Intern. Fis. Como., 2, 545-57 (1927).

${ }^{69}$ M. L. Bortz and R. H. French, "Quantitative, FFT-Based, Kramers Kronig Analysis for Reflectance Data," Appl. Spectrosc., 43 [8] 1498-501 (1989).

${ }^{70}$ D. Y. Smith, "Dispersion Theory, Sum Rules, and Their Application to the Analysis of Optical Data"; pp. 35-68 in Handbook of Optical Constants of Solids. Edited by E. D. Palik. Academic Press, New York, 1985.

${ }^{71}$ E. Shiles, T. Sasaki, M. Inokuti, and D. Y. Smith, "Self-Consistency and Sum-Rule Tests in the Kramers-Kronig Analysis of Optical Data: Applications to Aluminum," Phys. Rev. B: Condens. Matter, 22, 1612-26 (1980).

${ }^{72}$ R. F. Egerton, Electron Energy-Loss Spectroscopy in the Electron Microscope, 2nd ed.; p. 485. Plenum Press, New York, 1996.

${ }^{73}$ A. D. Dorneich, R. H. French, H. Müllejans, S. Loughin, and M. Rühle, "Quantitative Analysis of Valence Electron Energy-Loss Spectra of Aluminum Nitride," J. Microsc., 191 [3] 286-96 (1998).

${ }^{74}$ H. Müllejans and R. H. French, "Insights into the Electronic Structure of Ceramics through Quantitative Analysis of Valence Electron Energy-Loss Spectroscopy (VEELS)," Microsc. Microanaly., 6, 297-306 (2000).

${ }^{75}$ R. H. French and W. Y. Ching, "Ab Initio Calculation of Hamaker Constants of Silicon Oxides and Silicon Nitrides Using Lifshitz Theory," unpublished work.

${ }^{76}$ D. B. Hough and L. R. White, "The Calculation of Hamaker Constants from Lifshitz Theory with Application to Wetting Phenomena," Adv. Colloid Interface Sci., 14, 3-41 (1980).

${ }^{77}$ E. D. Palik (Ed.), Handbook of Optical Constants of Solids, Vol. I, 1985; Vol II, 1991; Vol. III, 1998. Academic Press, New York.

${ }^{78} \mathrm{~F}$. Gervais, "Aluminum Oxide $\left(\mathrm{Al}_{2} \mathrm{O}_{3}\right.$ "; pp. $761-75$ in Handbook of Optical Constants of Solids, Vol. II. Edited by E. D. Palik. Academic Press, New York, 1991. ${ }^{79}$ W. J. Tropf and M. E. Thomas, "Aluminum Oxide $\left(\mathrm{Al}_{2} \mathrm{O}_{3}\right)$ Revisited"; pp. 777-87 in Handbook of Optical Constants of Solids, Vol. III. Edited by E. D. Palik. Academic Press, New York, 1998.

${ }^{80}$ N. G. Van Kampen, B. R. A. Nijboer, and K. Schram, "On the Macroscopic Theory of van der Waals Forces," Phys. Lett., 26A, 307-308 (1968).

${ }^{81}$ D. C. Prieve and W. B. Russell, "Simplified Predictions of Hamaker Constants from Lifshitz Theory," J. Colloid Interface Sci., 125 [1] 1-13 (1988).

${ }^{82}$ L. Bergström, A. Meurk, H. Arwin, and D. J. Rowecliffe, "Estimation of Hamaker Constants of Ceramic Materials from Optical Data Using Lifshitz Theory," J. Am. Ceram. Soc., 79, 339-48 (1996).

${ }^{83}$ L. Bergström, S. Stemme, T. Dahlfors, H. Arwin, and L. Ödberg, "Spectroscopic Ellipsometry Characterisation and Estimation of the Hamaker Constant of Cellulose," Cellulose, 6, 1-13 (1999).

${ }^{84}$ V. A. Parsegian and G. H. Weiss, "Spectroscopic Parameters for Computation of van der Waals Forces," J. Colloid Interface Sci., 81, 285-89 (1981).

${ }^{85}$ C. M. Roth and A. M. Lenhoff, "Improved Parametric Representation of Water Dielectric Data for Lifshitz Theory Calculations," J. Colloid Interface Sci., 179, 637-39 (1996).

${ }^{86}$ R. M. Pashley, "The van der Waals Interaction for Liquid Water: A Comparison of the Oscillator Model Approximation and Use of the Kramers-Kronig Equation with Full Spectral Data," J. Colloid Interface Sci., 62, 344-47 (1977).

${ }^{87}$ H. D. Ackler, R. H. French, and Y. M. Chiang, "Comparison of Hamaker Constants for Ceramic Systems with Intervening Vacuum or Water: From Force Laws and Physical Properties," J. Colloid Interface Sci., 179, 460-69 (1996).

${ }^{88}$ M. R. Querry, D. M. Wieliczka, and D. J. Segelstein, "Water $\left(\mathrm{H}_{2} \mathrm{O}\right)$ "; see Ref. 78, pp. $1059-78$.

${ }^{89}$ O. V. Kopelevich, "Optical Properties of Pure Water in the 250-600 nm Range," Opt. Spektrosk., 41, 391-92 (1977).

${ }^{90}$ J. Lenoble, and B. Saint-Guilly, "Sur l'absorption du Rayonnement Ultraviolet par l'eau Distillée," Compt. Rend., 240, 954-55 (1955).

${ }^{91}$ L. R. Painter, R. D. Birkhoff, and E. T. Arakawa, "Optical Measurements of Liquid Water in the Vacuum Ultraviolet,” J. Chem. Phys., 51, 243-51 (1969).
${ }^{92}$ J. M. Heller Jr., R. N. Hamm, R. D. Birkhoff, and L. R. Painter, "Collective Oscillation in Liquid Water," J. Chem. Phys., 60, 3483-86 (1974).

${ }^{93}$ D. Tabor and R. H. S. Winterton, "The Direct Measurement of Normal and Retarded van der Waals Forces," Proc. R. Soc. London, A, 312, 435-50 (1969).

${ }^{94}$ J. N. Israelachvili, Eqs. 11.13 and 11.14 in Intermolecular and Surface Forces, 2nd ed.; p. 184. Academic Press, London, U.K., 1992.

${ }^{95}$ R. E. Johnson and R. H. Dettre, "Wetting of Low-Energy Surfaces"; pp. 1-73 in Wettability, Surfactant Science Series, Vol. 49. Edited by J. C. Berg. Marcel Dekker, New York, 1993.

${ }^{96}$ J. N. Israelachvili, "van der Waals Force Contribution to Works of Adhesion and Contact Angles on the Basis of Macroscopic Theory," J. Chem. Soc., Faraday Trans. 2, 69, 1729-38 (1974)

${ }^{97}$ K. Autumn, Y. A. Liang, S. T. Hsieh, W. Zesch, W. P. Chan, T. W. Kenny, R. Fearing, and R. I. Full, "Adhesive Force of a Single Gecko Foot-Hair," Nature (London), 405, 681-85 (2000)

${ }^{98}$ W. A. Zisman, "Relation of the Equilibrium Contact Angle to Liquid and Solid Constitution," Adv. Chem., 43, 1-51 (1964).

${ }^{99}$ R. J. Good and L. A. Girifalco, "A Theory for Estimation of Surface and Interfacial Energies, III, Estimation of Surface Energies of Solids from Contact Angle Data," J. Phys. Chem., 64, 561-65 (1960).

${ }^{100}$ D. R. Clarke and M. L. Gee, "Wetting of Surfaces and Grain Boundaries"; pp. 255-72 in Materials Interfaces. Chapman and Hall, London, U.K., 1992.

${ }^{101}$ D. R. Clarke, "Possible Electrical Double-Layer Contribution to the Equilibrium Thickness of Intergranular Glass Films in Polycrystalline Ceramics," J. Am. Ceram. Soc., 76, 1201-204 (1993).

${ }^{102}$ D. R. Clarke, "Wetting of Grain Boundaries in Metals and Ceramics"; pp. 1-8 in Intergranular and Interphase Boundaries in Materials, Materials Science Forum, Vol. 294-296. TransTech, Aedermannsdorf, Switzerland, 1999.

${ }^{103}$ J. W. Cahn, "Critical Point Wetting," J. Chem. Phys., 66 [8] 3667-72 (1977).

${ }^{104}$ J. W. Cahn and J. E. Hilliard, "Free Energy of a Nonuniform System. I. Interfacial Free Energy," J. Chem. Phys., 28, 258-67 (1958).

${ }^{105}$ S. Dietrich, "Fluid Interfaces: Wetting, Critical Adsorption, van der Waals Tails, and the Concept of the Effective Interface Potential"; pp. 391-423 in Phase Transitions in Surface Films, Vol. 2. Edited by H. Taub, G. Torzo, H. J. Lauter, and S. C. Fain Jr. Plenum Press, New York, 1991.

${ }^{106}$ Y. Solomentsev and L. R. White, "Microscopic Drop Profiles and the Origins of Line Tension," J. Colloid Interface Sci., 218, 122-36 (1999).

${ }^{107}$ M. Elbaum and S. G. Lipson, "How Does a Thin Wetted Film Dry Up?," Phys. Rev. Lett., 72, 3562-65 (1994).

${ }^{108}$ R. Yerushalmi-Rozen, J. Klein, and L. J. Fetters, "Suppression of Rupture in Thin, Nonwetting Liquid Films," Science (Washington), 263, 793-95 (1994).

${ }^{109} \mathrm{~S}$. Wu, Polymer Interface and Adhesion; pp. 87-94. Marcel Dekker, New York, 1982

${ }^{110}$ G. T. Dee and B. B. Sauer, "The Molecular Weight and Temperature Dependence of Polymer Surface Tension: Comparison of Experiment with Interface Gradient Theory," J. Colloid Interface Sci., 152, 85-103 (1992).

${ }^{111}$ J. A. Lewis, "Colloidal Processing of Ceramics," J. Am. Ceram. Soc., 83 [10] (2000), in press

${ }^{112}$ J. Th. G. Overbeek and M. J. Sparnaay, "I. Classical Coagulation: London-van der Waals Attraction Between Macroscopic Objects." Discuss. Faraday Soc., 18, 13-25 (1954).

${ }^{113}$ B. V. Derjaguin and L. Landau, "Theory of the Stability of Strongly Charged Lyophobic Sols and of the Adhesion of Strongly Charged Particles in Solutions of Electrolytes," Acta Phys. Chim. U.R.S.S., 14, 633 (1941).

${ }^{114}$ I. I. Abrikossova and B. V. Derjaguin, "Measurement of Molecular Attraction Between Solid Bodies of Different Nature at Large Distances"; pp. 398-405 in Proceedings of the Second International Congress on Surface Activity, Vol. 3 (London, U.K., 1957). Butterworths, London, U.K., 1957.

${ }^{115} \mathrm{~J}$. N Israelachvili, Intermolecular and Surface Forces, 2nd ed.; p. 169. Academic Press, London, U.K., 1992.

${ }^{116}$ R. G. Horn, D. R. Clarke, and M. T. Clarkson, "Direct Measurement of Surface Forces Between Sapphire Crystals in Aqueous Solutions," J. Mater. Res., 3, 413-16 (1988).

${ }^{117}$ J. N. Israelachvili and G. E. Adams, "Measurement of Forces Between Two Mica Surfaces in Aqueous Electrolyte Solutions in the Range 0-100 nm," J. Chem. Soc., 74, 975-1002 (1978).

${ }^{118}$ R. G. Horn and J. N. Israelachvili, "Direct Measurement of Forces due to Solvent Structure," Chem. Phys. Lett., 71, 192-94 (1980).

${ }^{119}$ R. M. Pashley and J. N. Israelachvili, "Molecular Layering of Water in Thin Films Between Mica Surfaces and Its Relation to Hydration Forces," J. Colloid Interface Sci., 101, 511-23 (1984).

${ }^{120}$ G. Binnig, C. F. Quate, and Ch. Gerber, "Atomic Force Microscope," Phys. Rev. Lett., 56, 930-33 (1985)

${ }^{121}$ A. L. Weisenhorn, P. K. Hansma, T. R. Albrecht, and C. F. Quate, "Forces in Atomic Force Microscopy in Air and Water," Appl. Phys. Lett., 54, 2651-53 (1989).

${ }^{122}$ W. A. Ducker, T. J. Senden, and R. M. Pashley, "Measurements of Forces in Liquids Using a Force Microscope," Langmuir, 8 [7] 1831-36 (1992)

${ }^{123}$ T. J. Senden, C. J. Drummond, and P. Kékicheff, "Atomic Force Microscopy: Imaging with Electrical Double Layer Interactions," Langmuir, 10, 358-62 (1994).

${ }^{124}$ U. Hartmann, "Theory of van der Waals Microscopy," J. Vac. Sci. Technol. B, 9, 465-69 (1991).

${ }^{125}$ C. Argento and R. H. French, "Parametric Tip Model and Force-Distance Relation for Hamaker Constant Determination from AFM," J. Appl. Phys., 80, 6081-90 (1996)

${ }^{126}$ W. A. Ducker and D. R. Clarke, "Controlled Modification of Silicon Nitride Interactions in Water Via Zwitterionic Surfactant Adsorption," Colloids Surf. A, 94, 275-92 (1994).

${ }^{127}$ I. Larson, C. J. Drummond, D. Y. C. Chan, and F. Grieser, "Direct Force Measurements Between $\mathrm{TiO}_{2}$ Surfaces," J. Am. Chem. Soc., 115 [25] 11885-90 (1993). 
${ }^{128}$ S. Biggs and P. Mulvaney, "Measurement of the Forces Between Gold Surfaces in Water by Atomic Force Microscopy," J. Chem. Phys., 100, 8501-505 (1994).

${ }^{129}$ W. A. Ducker, Z. Xu, D. R. Clarke, and J. N. Israelachvili, "Forces Between Alumina Surfaces in Salt Solutions: Non-DLVO Forces and the Implications for Colloidal Processing," J. Am. Ceram. Soc., 77, 437-43 (1994).

${ }^{130}$ M. S. Hook, P. G. Hartley, and P. J. Thistlethwaite, "Fabrication and Characterization of Spherical Zirconia Particles for Direct Force Measurements Using the Atomic Force Microscope," Langmuir, 15, 6220-25 (1999).

${ }^{131}$ H. G. Pedersen and L. Bergström, "Forces Measured Between Zirconia Surfaces in Poly(acrylic acid) Solutions," J. Am. Ceram. Soc., 82, 1137-45 (1999).

${ }^{132} \mathrm{H}$. Bluhm, T. Inoue, and M. Salmeron, "Friction of Ice Measured Using Lateral Force Microscopy," Phys. Rev. B: Condens. Matter, 61, 7760-65 (2000).

${ }^{133} \mathrm{G}$. I. Barenblatt, "The Mathematical Theory of Equilibrium Cracks in Brittle Fracture," Adv. Appl. Mech., 7, 55-129 (1962).

${ }^{134}$ A. Jagota, C. Argento, and S. Mazur, "Growth of Adhesive Contacts for Maxwell Viscoelastic Spheres," J. Appl. Phys., 83 [1] 250-59 (1998).

${ }^{135}$ C. Argento, A. Jagota, and W. C. Carter, "Surface Formulation for Molecular Interactions of Macroscopic Bodies," J. Mech. Phys. Solids, 45 [7] 1161-83 (1997).

${ }^{136}$ A. W. Adamson, Physical Chemistry of Surfaces, 3rd ed.; Ch. 1. Wiley, New York, 1976.

${ }^{137}$ V. B. Bezerra, G. L. Klimchitskaya, and C. Romero, "Casimir Force Between a Flat Plate and a Spherical Lens: Applications to the Results of a New Experiment," Modern Phys. Lett. A, 12, 2613-22 (1997).

${ }^{138} \mathrm{~B}$. V. Derjaguin, "Untersuchungen über die Reibung und Adhesion, IV. Theorie des Anhaftens kleiner Teilchen," Kolloid-Z., 69, 155-64 (1934).

${ }^{139}$ A. Jagota and C. Argento, "An Intersurface Stress Tensor," J. Colloid Interface Sci., 191, 326-36 (1997).

${ }^{140}$ R. M. McMeeking, "A Maxwell Stress for Material Interactions," J. Colloid Interface Sci., 199, 187-96 (1998).

${ }^{141}$ J. D. Jackson, Classical Electrodynamics, 2nd ed. Wiley, New York, 1975. (1974).

${ }^{143}$ F. B. Swinkels and M. F. Ashby, "A Second Report on Sintering Diagrams," Acta Metall., 29, 259-81 (1981).

${ }^{144} \mathrm{~K}$. L. Johnson, K. Kendall, and A. D. Roberts, "Surface Energy and the Contact of Elastic Solids," Proc. R. Soc. London, A, 324, 301-13 (1971).

${ }^{145}$ J. Eggers, "Coalescence of Spheres by Surface Diffusion," Phys. Rev. Lett., 80, 2634-37 (1998).

${ }^{146}$ G. C. Kuczynski, "Study of the Sintering of Glass," J. Appl. Phys., 20, 1160-63 (1949).

${ }^{147}$ C. Herring, "Effect of Change of Scale on Sintering Phenomena," J. Appl. Phys., 21, 301-303 (1950)

${ }^{148}$ S. Mazur and D. J. Plazek, "Viscoelastic Effects in the Coalescence of Polymer Particles," Prog. Org. Coatings, 24, 225-36 (1994).

${ }^{149}$ R. M. Cannon and L. Esposito, "High-Temperature Colloidal Behavior: Particles in Liquid Silicates," Z. Metallkd., 90, 1002-15 (1999).

${ }^{150} \mathrm{~L}$. Esposito, A. Bellosi, and S. Landi, "Interfacial Forces in $\mathrm{Si}_{3} \mathrm{~N}_{4^{-}}$and SiC-Based Systems and Their Influence on the Joining Process," J. Am. Ceram. Soc., 82, 3597-604 (1999).

${ }^{151}$ J. White, "Microstructure and Grain Growth in Ceramics in the Presence of a Liquid Phase"; pp. 81-108 in Sintering and Related Phenomena. Edited by G. C. Kuczynski. Plenum Press, New York, 1973.

${ }^{152}$ W. A. Kaysser, M. Sprissler, C. A. Handwerker, and J. E. Blendell, "Effect of a Liquid Phase on the Morphology of Grain Growth in Alumina," J. Am. Ceram. Soc., 70 [5] 339-43 (1987).

${ }^{53}$ M. J. Hoffmann, "Analysis of Microstructure Development and Mechanical Properties of $\mathrm{Si}_{3} \mathrm{~N}_{4}$ Ceramics"; pp. 59-72 in Tailoring of Mechanical Properties of $\mathrm{Si}_{3} \mathrm{~N}_{4}$ Ceramics. Edited by M. J. Hoffmann and G. Petzow. Kluwer, Dordrecht, The Netherlands, 1994

${ }^{154}$ W. D. Kingery, "Distribution and Influence of Minor Constituents on Ceramic Formulations"; pp. 281-94 in Ceramic Microstructures '86: Role of Interfaces. Edited by J. A. Pask and A. G. Evans. Plenum Press, New York, 1987.

${ }^{155} \mathrm{H}$.-J. Kleebe, "Structure and Chemistry of Interfaces in $\mathrm{Si}_{3} \mathrm{~N}_{4}$ Materials Studied by Transmission Electron Microscopy," J. Ceram. Soc. Jpn., 105 [6] 453-75 (1997),

${ }^{156} \mathrm{R}$. Raj, "Fundamental Research in Structural Ceramics for Service Near $2000^{\circ}$ C, J. Am. Ceram. Soc., 76 [9] 2147-74 (1993).

${ }^{157}$ S. M. Wiederhorn, B. J. Hockey, and J. D. French, "Mechanisms of Deformation of Silicon Nitride and Silicon Carbide at High Temperature," J. Eur. Ceram. Soc., 19, 2273-84 (1999).

${ }^{158}$ P. F. Becher, E. Y. Sun, K. P. Plucknett, K. B. Alexander, C.-H. Hsueh, H-T. Lin, S. B. Waters, and C. G. Westmoreland, "Microstructural Design of Silicon Nitride with Improved Fracture Toughness: I, Effects of Grain Shape and Size," J. Am. Ceram. Soc., 81 [11] 2821-30 (1998).

${ }^{159}$ E. Y. Sun, P. F. Becher, K. P. Plucknett, C.-H. Hsueh, K. B. Alexander, S. B. Waters, K. Hirao, and M. E. Brito, "Microstructural Design of Silicon Nitride with Improved Fracture Toughness: II, Effects of Yttria and Alumina Additions," J. Am. Ceram. Soc., 81 [11] 2831-40 (1998).

${ }^{160}$ (a)N. P. Padture, "In Situ-Toughened Silicon Carbide," J. Am. Ceram. Soc., 77 [2] 519-23 (1994). (b) K. M. Knowles and S. Turan, "The Dependence of Equilibrium Film Thickness on Grain Orientation at Interphase Boundaries in Ceramic-Ceramic Composites," Ultramicroscopy, 83, 245-59 (2000).

${ }^{161}$ J. J. Cao, W. J. Moberly-Chan, L. C. DeJonghe, C. J. Gilbert, and R. O. Ritchie, "In Situ-Toughened Silicon Carbide with Al-B-C Additions," J. Am. Ceram. Soc., 79 [2] 461-69 (1996)

${ }^{162}$ K. M. Knowles, S. Turan, A. Kumar, S.-J. Chen, and W. J. Clegg, "Transmission Electron Microscopy of Interfaces in Structural Ceramic Composites," J. Microsc., 196 [Part 2] 194-202 (1999).

${ }^{163}$ Y.-M. Chiang, L. A. Silverman, R. H. French, and R. M. Cannon, "Thin Glass Film between Ultrafine Conductor Particles in Thick-Film Resistors," J. Am. Ceram. Soc., 77 [5] 1143-52 (1994).
${ }^{164} \mathrm{~F}$. Greuter, "Electrically Active Interfaces in $\mathrm{ZnO}$ Varistor," Solid State Ionics, 75 [1] 67-78 (1995).

${ }^{165}$ R. Ramesh, S. M. Green, and G. Thomas, "Microstructure Property Relations in the $\mathrm{Bi}(\mathrm{Pb})-\mathrm{Sr}-\mathrm{Ca}-\mathrm{Cu}-\mathrm{O}$ Ceramic Superconductors"; pp. 363-403 in Studies of High-Temperature Superconductors, Advances in Research and Applications, Vol. 5. Nova Science Publications, Commack, NY, 1990.

${ }^{166} \mathrm{H}$. Gu, R. M. Cannon, and M. Rühle, "Composition and Chemical Width of Ultra-Thin Intergranular Amorphous Film in Silicon Nitride," J. Mater. Res., 13 [2] 476-87 (1998).

${ }^{167} \mathrm{H}$. Gu, X. Pan, R. M. Cannon, and M. Rühle, "Dopant Distribution in Grain-Boundary Films in Calcia-Doped Silicon Nitride Ceramics," J. Am. Ceram. Soc., 81 [12] 3125-35 (1998).

${ }^{168} \mathrm{H}$. Gu, R. M. Cannon, H. J. Seifert, and M. J. Hoffmann, "Solubility of $\mathrm{Si}_{3} \mathrm{~N}_{4}$ in Liquid $\mathrm{SiO}_{2}$, , J. Am. Ceram. Soc., in review.

${ }^{169}$ I. Tanaka, H. Adachi, T. Nakayasu, and T. Yamada, "Local Chemical Bonding at Grain Boundary of $\mathrm{Si}_{3} \mathrm{~N}_{4}$ Ceramics"; pp. 23-34 in Ceramic Microstructure: Control at the Atomic Level. Edited by A. P. Tomsia and A. Glaeser. Plenum Press, New York, 1998.

${ }^{170}$ Y.-M. Chiang, J.-R. Lee, and H. Wang, "Microstructure and Intergranular Phase Distribution in $\mathrm{Bi}_{2} \mathrm{O}_{3}$-Doped $\mathrm{ZnO";} \mathrm{see} \mathrm{Ref.} \mathrm{172,} \mathrm{pp.} \mathrm{131-47.}$

${ }^{171}$ R. Brydson, S.-C. Chen, F. L. Riley, S. J. Milne, X. Pan, and M. Rühle, "Microstructure and Chemistry of Intergranular Glassy Films in Liquid-PhaseSintered Alumina," J. Am. Ceram. Soc., 81 [2] 369-79 (1998).

${ }^{172}$ G. H. Gessinger, H. F. Fischmeister, and H. L. Lukas, "A Model for Second-Stage Liquid-Phase Sintering with a Partially Wetting Liquid," Acta Metall., 21 [5] 715-24 (1973).

${ }^{173}$ H.-H. Park, S.-J. L. Kang, and D. N. Yoon, "An Analysis of the Surface Menisci in a Mixture of Liquid and Deformable Grains," Metall. Trans., 17A [2] 325-30 (1986)

${ }^{174}$ T. M. Shaw, "Model for the Effect of Powder Packing on the Driving Force for Liquid-Phase Sintering," J. Am. Ceram. Soc., 76 [3] 664-70 (1993).

${ }^{175} \mathrm{~J}$. Luo, H. Wang, and Y.-M. Chiang, "Origin of Solid-State Activated Sintering in $\mathrm{Bi}_{2} \mathrm{O}_{3}$-Doped $\mathrm{ZnO}$," J. Am. Ceram. Soc., 82 [4] 916-20 (1999).

${ }^{176}$ D. R. Clarke and G. Thomas, "Grain Boundary Phases in a Hot-Pressed $\mathrm{MgO}$ Fluxed Silicon Nitride," J. Am. Ceram. Soc., 60 [11-12] 491-95 (1977).

${ }^{177}$ L. K. V. Lou, T. E. Mitchell, and A. H. Heuer, "Impurity Phases in Hot-Pressed $\mathrm{Si}_{3} \mathrm{~N}_{4}$, J. Am. Ceram. Soc., 61 [9-10] 392-96 (1978).

${ }^{178}$ O. L. Krivanek, T. M. Shaw, and G. Thomas, "The Microstructure and Distribution of Impurities in Hot-Pressed and Sintered Silicon Nitrides," J. Am. Ceram. Soc., 62 [11-12] 585-90 (1979).

${ }^{179}$ H.-J. Kleebe, M. K. Cinibulk, R. M. Cannon, and M. Rühle, "Statistical Analysis of the Intergranular Film Thickness in Silicon Nitride Ceramics," J. Am. Ceram. Soc., 76 [8] 1969-77 (1993).

${ }^{180}$ I. Tanaka, H.-J. Kleebe, M. K. Cinibulk, J. Bruley, D. R. Clarke, and M. Rühle, "Calcium Concentration Dependence of the Intergranular Film Thickness in Silicon Nitride," J. Am. Ceram. Soc., 77, 911-14 (1994).

${ }^{181}$ C.-M. Wang, X. Pan, M. J. Hoffmann, R. M. Cannon, and M. Rühle, "Grain Boundary Films in Rare-Earth-Glass-Based Silicon Nitride," J. Am. Ceram. Soc., 79 [3] 788-92 (1996).

${ }^{182}$ D. R. Clarke, "On the Equilibrium Thickness of Intergranular Glassy Phases in Ceramic Materials," J. Am. Ceram. Soc., 70 [1] 15-22 (1987).

${ }^{183}$ D. R. Clarke, T. M. Shaw, A. Philipse, and R. G. Horn, "Possible Electrical Double-Layer Contribution to the Equilibrium Thickness of Intergranular Glass Phases in Polycrystalline Ceramics," J. Am. Ceram. Soc., 76 [5] 1201-204 (1994).

${ }^{184} \mathrm{H}$. Wang and Y.-M. Chiang, "Thermodynamic Stability of Intergranular Amorphous Films in Bismuth-Doped Zinc Oxide," J. Am. Ceram. Soc., 81 [1] 89-96 (1998).

${ }^{185}$ T. M. Shaw and P. R. Duncombe, "Forces between Aluminum Oxide Grains in a Silicate Melt and Their Effect on Grain Boundary Wetting," J. Am. Ceram. Soc., 74 [10] 2495-505 (1991)

${ }^{186}$ P. L. Flaitz and J. A. Pask, "Penetration of Polycrystalline Alumina by Glass at High Temperatures," J. Am. Ceram. Soc., 70 [7] 449-55 (1987).

${ }^{87} \mathrm{~J}$.-R. Lee and Y.-M. Chiang, "Bi Segregation at $\mathrm{ZnO}$ Grain Boundaries in Equilibrium with $\mathrm{Bi}_{2} \mathrm{O}_{3}-\mathrm{ZnO}$ Liquid," Solid State Ionics, 75, 79-88 (1995).

${ }^{188}$ L. Esposito, E. Saiz, A. P. Tomsia, and R. M. Cannon, "High-Temperature Colloidal Processing for Glass/Metal and Glass/Ceramic FGM's"; pp. 503-12 in Ceramic Microstructure: Control at the Atomic Level. Edited by A. P. Tomsia and A. Glaeser. Plenum Press, New York, 1998.

${ }^{189} \mathrm{~T}$. H. Courtney, "Densification and Structural Development in Liquid Phase Sintering," Metall. Trans A., 15A, 1065-74 (1984).

${ }^{190}$ J. Jitcharoen, N. Padture, A. E. Giannakopoulos, and S. Suresh, "Hertzian-Crack Suppression in Ceramics with Elastic-Modulus Graded Surfaces," J. Am. Ceram. Soc., 81 [9] 2301-308 (1998).

${ }^{191}$ D. C. Pender, "Ceramics with Graded Surfaces for Contact Damage Resistance"; Ph.D. Dissertation. University of Connecticut, Storrs, CT, 1999.

${ }^{192} \mathrm{~L}$. Esposito and A. Bellosi, "The Role of Intergranular Phases in $\mathrm{Si}_{3} \mathrm{~N}_{4}$ and $\mathrm{SiC}$ Direct Joining"; pp. 211-22 in Interfacial Science in Ceramic Joining. Edited by A. Bellosi, T. Kosmac, and A. P. Tomsia. Kluwer, Boston, MA, 1998.

${ }^{193}$ M. Gopal, L. C. DeJonghe, and G. Thomas, "Silicon Nitride: From Sintering to Joining," Acta Mater., 46 [7] 2401-405 (1998).

${ }^{194}$ Y. K. Simpson, C. B. Carter, K. J. Morrissey, P. Angelini, and J. Bentley, "The Identification of Thin Amorphous Films at Grain Boundaries in $\mathrm{Al}_{2} \mathrm{O}_{3}$," J. Mater. Sci., 21, 2689-96 (1986).

${ }^{195} \mathrm{M}$. Rühle, N. Claussen, and A. H. Heuer, "Microstructural Studies of $\mathrm{Y}_{2} \mathrm{O}_{3-}$ Containing Tetragonal $\mathrm{ZrO}_{2}$ Polycrystals (Y-TZP)," Adv. Ceram., 12, 352-70 (1984).

${ }^{196}$ L. K. V. Lou, T. E. Mitchell, and A. H. Heuer, "Impurity Phases in Hot-Pressed $\mathrm{Si}_{3} \mathrm{~N}_{4}$, J. J. Am. Ceram. Soc., 61, 392-96 (1978).

${ }^{197}$ D. L. Callahan and G. Thomas, "Impurity Distribution in Polycrystalline Aluminum Nitride Ceramics," J. Am. Ceram. Soc., 73, 2167-70 (1990). 
${ }^{198}$ F. F. Lange and D. R. Clarke, "Morphological Changes of an Intergranular Thin Film in a Polycrystalline Spinel," J. Am. Ceram. Soc., 65, 502-506 (1982).

${ }^{199}$ S. C. Hansen and D. S. Phillips, "Grain-Boundary Microstructures in a Liquid-Phase-Sintered Alumina $\left(\alpha-\mathrm{Al}_{2} \mathrm{O}_{3}\right)$," Philos. Mag. A, A47 [2] 209-34 (1983).

${ }^{200}$ D. W. Susnitzky and C. B. Carter, "Structure of Alumina Grain Boundaries Prepared with and without a Thin Amorphous Intergranular Film," J. Am. Ceram. Soc., 73 [8] 2485-93 (1990).

${ }^{201}$ C. A. Powell-Dogan and A. H. Heuer, "Microstructure of $96 \%$ Alumina Ceramics: I, Characterization of the As-Sintered Materials," J. Am. Ceram. Soc., 73 [12] 3670-76 (1990).

${ }^{202}$ D.-Y. Kim, S. M. Wiederhorn, B. J. Hockey, J. E. Blendell, and C. A. Handwerker, "Stability and Surface Energies of Wetted Grain Boundaries in Aluminum Oxide," J. Am. Ceram. Soc., 77 [2] 444-53 (1994).

${ }^{203}$ J. E. Blendell, W. C. Carter, and C. A. Handwerker, "Faceting and Wetting Transitions of Anisotropic Interfaces and Grain Boundaries," J. Am. Ceram. Soc., 82 [7] 1889-900 (1999).

${ }^{204}$ P. L. Flaitz and J. A. Pask, "Penetration of Polycrystalline Alumina by Glass at High Temperatures," J. Am. Ceram. Soc., 70 [7] 449-55 (1987).

${ }^{205}$ T. M. Shaw and P. R. Duncombe, "Forces between Aluminum Oxide Grains in a Silicate Melt and Their Effect on Grain Boundary Wetting," J. Am. Ceram. Soc., 74 [10] 2495-505 (1991).

${ }^{206}$ S. Ramamurthy, M. P. Mallamaci, C. M. Zimmerman, C. B. Carter, P. R. Duncombe, and T. M. Shaw, "Microstructure of Polycrystalline MgO Penetrated by a Silicate Liquid," JMSA ISSN, 2 [3] 113-28 (1996).

${ }^{207}$ F. Ernst, O. Kienzle, and M. Rühle, "Structure and Composition of Grain Boundaries in Ceramics," J. Eur. Ceram. Soc., 19, 665-73 (1999).

${ }^{208}$ O. Kienzle, "Atomistische Struktur und Chemische Zusammensetzung innerer Grenzflächen von Strontiumtitanat"; Ph.D. Dissertation. University of Stuttgart and Max-Planck Institute for Metals Research, Stuttgart, Germany, 1999.

${ }^{209}$ J. P. Gambino, W. D. Kingery, G. E. Pike, and H. R. Phillip, "Effect of Heat Treatments on the Wetting Behavior of Bismuth-Rich Intergranular Phases in ZnO:Bi:Co Varistors," J. Am. Ceram. Soc., 72, 642-45 (1989).

${ }^{210} \mathrm{E}$. Olsson and G. L. Dunlop, "The Effect of $\mathrm{Bi}_{2} \mathrm{O}_{3}$ Content on the Microstructure and Electrical Properties of ZnO Varistor Materials," J. Appl. Phys., 66, 4317-24 (1989).

${ }^{211}$ M. Fujimoto and W. D. Kingery, "Microstructures of $\mathrm{SrTiO}_{3}$ Internal Boundary Layer Capacitors During and After Processing and the Resultant Electrical Properties," J. Am. Ceram. Soc., 68 [4] 169-73 (1985).

${ }^{212}$ M. Kramer, M. J. Hoffmann, and G. Petzow, "Grain Growth Studies of Silicon Nitride Dispersed in an Oxynitride Glass," J. Am. Ceram. Soc., 76, 2778-84 (1993).

${ }^{213}$ I. Tanaka, J. Bruley, H. Gu, M. J. Hoffmann, H. J. Kleebe, R. M. Cannon, D. R. Clarke, and M. Rühle; pp. 275-89 in Tailoring of Mechanical Properties of $\mathrm{Si}_{3} \mathrm{~N}_{4}$ Ceramics. Kluwer Academic Publishers, Stuttgart, Germany, 1994.

${ }^{214}$ J.-R. Lee, Y.-M. Chiang, and G. Ceder, "Pressure-Thermodynamic Study of Grain Boundaries: Bi Segregation in ZnO," Acta Mater., 45[3] 1247-57 (1997).

${ }^{215} \mathrm{H}$. Wang and Y.-M. Chiang, "Thermodynamic Stability of Intergranular Amorphous Films in Bi-Doped ZnO," J. Am. Ceram. Soc., 81[1] 89-96 (1998).

${ }^{216}$ Y.-M. Chiang, H. Wang, and J.-R. Lee, "HREM and STEM of Intergranular Films at Zinc Oxide Varistor Grain Boundaries," J. Microsc., 191 [3] 275-85 (1998).
${ }^{217}$ J. Luo, H. Wang, and Y.-M. Chiang, "Origin of Solid-State Activated Sintering in $\mathrm{ZnO}-\mathrm{Bi}_{2} \mathrm{O}_{3}$," J. Am. Ceram. Soc., 82 [4] 916-20 (1999).

${ }^{218}$ T. D. Chen, J.-R. Lee, H. L. Tuller, and Y.-M. Chiang, "Dopant and Heat-Treatment Effects on the Electrical Properties of Polycrystalline ZnO"; pp. 295-300 in Electrically Based Microstructural Characterization, Materials Research Society Symposium Proceedings, Vol. 41. Materials Research Society, Pittsburgh, PA, 1996.

${ }^{219}$ J. Luo and Y.-M. Chiang, "Equilibrium Thickness Amorphous Films on $\{11 \overline{2} 0\}$ Surfaces of $\mathrm{Bi}_{2} \mathrm{O}_{3}$-Doped $\mathrm{ZnO}$, J. Eur. Ceram. Soc., 19, 697-701 (1999).

${ }^{220}$ J. Juo and Y.-M. Chiang, "Existence and Stability of Nanometer-Thick Amorphous Films on Oxide Surfaces," Acta Mater., in review.

${ }^{221}$ V. A. Parsegian and G. H. Weiss, "Dielectric Anisotropy and the van der Waals Interaction Between Bulk Media," J. Adhes., 3, 259-67 (1972).

${ }^{222}$ V. A. Parsegian and G. H. Weiss, "On van der Waals Interactions Between Macroscopic Bodies Having Inhomogeneous Dielectric Susceptibilities," J. Colloid Interface Sci., 40, 35-41 (1972).

${ }^{223}$ H. D. Ackler, W. C. Carter, and Y.-M. Chiang, unpublished work

${ }^{224}$ M. Bobeth, D. R. Clarke, and W. Pompe, "A Diffuse Interface Description of Intergranular Films in Polycrystalline Ceramics," J. Am. Ceram. Soc., 82, 1537-46 (1999).

${ }^{225}$ J. A. Warren, W. C. Carter, and R. Kobayashi, "A Phase Field Model of the Impingement of Solidifying Particles," Physica A (Amsterdam), 14, 8 (1998).

${ }^{226}$ D. A. Muller, T. Sorsch, S. Moccio, F. H. Baumann, K. Evans-Lutterodt, and G. Timp, "The Electronic Structure at the Atomic Scale of Ultrathin Gate Oxides," Nature (London), 399, 758-61 (1999).

${ }^{227}$ N. A. Modine, G. Zumbach, and E. Kaxiras, "Adaptive-Coordinate Real-Space Electronic-Structure Calculations for Atoms, Molecules, and Solids," Phys. Rev. B: Condens. Matter, 55, 10289-301 (1997).

${ }^{228}$ J. S. Wettlaufer, "Impurity Effects in the Premelting of Ice," Phys. Rev. Lett., 82, 2516-19 (1999).

${ }^{229}$ R. Bar-Ziv and S. A. Safran, "Surface Melting of Ice Induced by Hydrocarbon Films," Langmuir, 9, 2786-88 (1993).

${ }^{230}$ J. Brandrup and E. H. Immergut, The Polymer Handbook, 3rd ed. Wiley Interscience, New York, 1989.

${ }^{231}$ A. Souter, DuPont Packaging and Industrial Polymers, private communication. ${ }^{232}$ C. A. Harper, Handbook of Plastics and Elastomers. McGraw-Hill, New York, 1975 .

${ }^{233}$ (a)S. Wu, Polymer Interface and Adhesion; pp. 87-94. Marcel Dekker, New York, 1982. (b)S. Wu, "Surface and Interfacial Tensions of Polymers, Oligomers, Plasticizers, and Organic Pigments"; pp. 411-34 in Polymer Handbook, 3rd ed. Wiley Interscience, New York, 1989.

${ }^{234}$ R. H. French, C. Scheu, G. Duscher, H. Müllejans, M. J. Hoffmann, and R. M. Cannon, "Interfacial Electronic Structure and Full Spectral Hamaker Constants of $\mathrm{Si}_{3} \mathrm{~N}_{4}$ Intergranular Films from VUV and SR-VEEL Spectroscopy," Mater. Res. Soc. Symp. Proc., 357, 243-58 (1995).

${ }^{235}$ H. D. Ackler and Y.-M. Chiang, "Effect of Initial Microstructure on Final Intergranular Phase Distributions in Liquid-Phase-Sintered Ceramics," J. Am. Ceram. Soc., 82 [1] 183-89 (1999). 


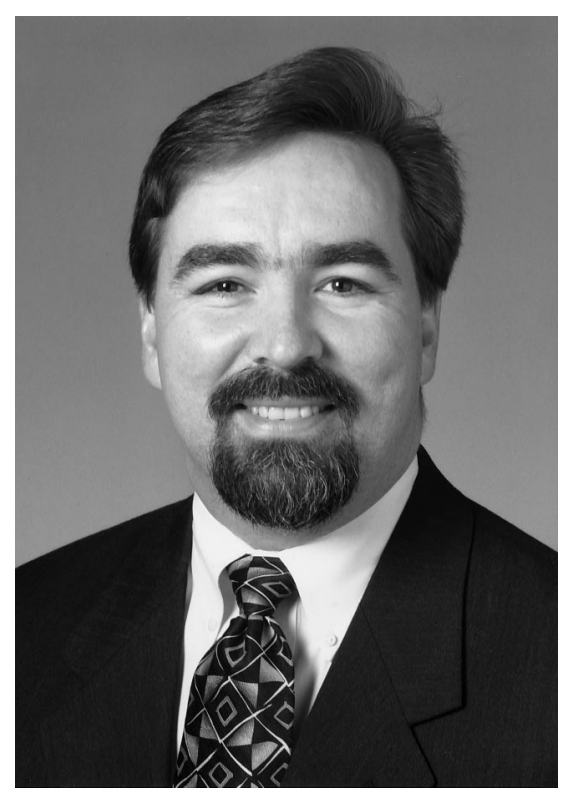

Roger H. French is a Senior Research Associate, Materials Science and Engineering, Central Research and Development, DuPont Company, Wilmington, DE, and Adjunct Professor, Materials Science Department, University of Pennsylvania, Philadelphia. He received his B.S. in materials science from Cornell University and his Ph.D. from Massachusetts Institute of Technology under R. L. Coble. French's research is in optical properties and electronic structure of ceramic, optical, and polymeric materials, and in materials and microstructure design. These studies are pursued using vacuum ultraviolet spectroscopy, spectroscopic ellipsometry, valence-electron energy-loss spectroscopy, and computational optics. His current work is in the origins and applications of London dispersion forces and the electronic structure and wetting of interfaces and intergranular films. French studies near-field optics and scattering by particulate dispersions and complex microstructures using computational solutions to Maxwell's equations. Work on new materials for optical lithography for integrated chip fabrication at 365, 248, 193, and $157 \mathrm{~nm}$ wavelengths has produced attenuating phase shift photomasks, photomask pellicles, and semiconductor photoresists. His work has produced nine patents and filings and more than 85 published papers. French is a Director of The American Ceramic Society, past chair of the Basic Science Division, and an Associate Editor of the Journal of the American Ceramic Society. He has served as co-chair for the Basic Science Division Program Committee and Gordon Conference on Solid-State Studies in Ceramics. French is a Fulrath awardee and a Fellow of The American Ceramic Society. 